\title{
DEVELOPMENT OF NEW METHODS IN FLUORESCENCE MICROSCOPY
}

\author{
Dissertation \\ for the award of the degree "Doctor rerum naturalium" \\ of the Georg-August-Universität Göttingen
}

within the doctoral program IMPRS Physics of Biological and Complex Systems of the Georg-August University School of Science (GAUSS)

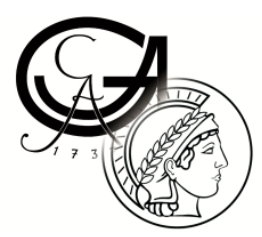

submitted by

Chao-Chen Lin

from Taipei, Taiwan

\section{Göttingen}

November 2015 



\section{Thesis Committee}

\section{Prof. Dr. Peter Jomo Walla (Referee)}

Research Group Biomolecular Spectroscopy and Single-Molecule Detection, Max Planck Institute for Biophysical Chemistry

and

Department of Biophysical Chemistry, Institute for Physical and Theoretical Chemistry, Technical University of Braunschweig

\section{Prof. Dr. Reinhard Jahn (Referee)}

Department of Neurobiology, Max Planck Institute for Biophysical Chemistry

\section{Prof. Dr. Claudia Steinem}

Institute for Organic and Biomolecular Chemistry, Georg-August-University Göttingen

\section{Members of the Examination Board}

\section{Prof. Dr. Claudia Höbartner}

Research Group Nucleic Acid Chemistry, Max Planck Institute for Biophysical Chemistry and Institute for Organic and Biomolecular Chemistry, Georg-August-University Göttingen

\section{Prof. Dr. Eberhard Bodenschatz}

Laboratory for Fluid Dynamics, Pattern Formation and Biocomplexity, Max Planck Institute for Dynamics and Self-Organization and Institute for Nonlinear Dynamics, Georg-August-University Göttingen

\section{Prof. Dr. Jörg Enderlein}

III. Institute of Physics, Georg-August-University Göttingen 
To dear grandma, aunt and Mrs. Kao 


\section{DECLARATION}

This dissertation is the result of my own work and includes nothing, which is the outcome of work done in collaboration except where specifically indicated in the text. It has not been previously submitted, in part or whole, to any university of institution for any degree, diploma, or other qualification.

I take full responsibilities for reusing materials in my own work, published under a Creative Commons Attribution 4.0 International License (CC BY), when I felt that any changes would make them inferior. The detailed attribution is given in Section 3.1.

Signed:

Date:

Chao-Chen Lin 


\section{SUMMARY / ABSTRACT}

In recent years, advancements in single-molecule imaging techniques have enabled scientists to study in great detail the cells and relevant physiological processes, including neurons and the communication between them. Nevertheless, with proper experimental design, much can still be learned from conventional fluorescence spectroscopy. In this work I use primarily the fluorescence lifetime as an indicator of FRET (Förster resonance energy transfer), which gives information on the interaction between liposomes.

The dissertation consists of two connected projects, and in each a new chemical tool is developed. In the first part, control of membrane gaps by synaptotagmin-Ca ${ }^{2+}$ measured with a novel membrane distance ruler, a set of liposomes bridged by double-stranded DNAs of various lengths serves as the molecular ruler to measure the changes in membrane distances induced by binding of $\mathrm{Ca}^{2+}$ to synaptotagmin-1 (syt-1). I showed that the distance maintained by syt-1 alone was reduced by one-third from $\sim 7-8 \mathrm{~nm}$ to $\sim 5 \mathrm{~nm}$, which may explain how syt-1 functions as the fast and efficient $\mathrm{Ca}^{2+}$ trigger in promoting the zippering of SNARE proteins, which leads subsequently to membrane fusion and neurotransmitter release. The major part of this project has been published in Nat. Commun. 2014, 5, 5859 (doi: 10.1038/ncomms6859).

In the second part, asymmetrically labeled liposomes as a new tool to study membrane fusion, one type of liposomes is labeled differently on the inner and outer leaflets, and the two fluorescent labels are distinguished by their differently lifetimes. Theses liposomes were used to monitor SNARE-mediated membrane fusion in microfluidic channels, and it turned out that there was no apparent delay between the merging of the two leaflets.

As a final remark, further characterization and improvements of the two new tools should allow for their future applications in studying other cellular mechanisms of interest. 


\section{ACKNOWLEDGEMENTS}

First of all, thanks to Jomo for giving me the opportunity to work at MPI-BPC and to apply fluorescence spectroscopy to understand a little bit more about how neurons communicate with each other. It's really enjoyable to work in a lab together with Matthias, Sabrina, Jian-Hua, Wensi and Iman. Additionally, Inge, Julia, Antje, Frauke and Tina have all helped me a great deal to cope with administrative matters.

Secondly, I am very grateful to Reinhard for the invaluable advices on the projects and Angel for providing the proteins and the helpful discussions. Furthermore, I would like to thank Claudia (Prof. Claudia Höbartner) and Jan for realizing the first part of my thesis work. Prof. Claudia Steinem has also given me many critical suggestions in the thesis committee meetings. Many thanks to Prof. Bodenschatz and Prof. Enderlein for being on my examination board.

Most importantly, this work could not be completed without the support from Hsin-Fang, with whom I have spent three and a half years here in Göttingen and four and a half years back in Taiwan. She happens to be the indispensable collaborator in the second part of my work. Lastly, my parents have always made me feel their warm care overseas, and I thereby dedicate my dissertation to them as well.

Thank you to Kayla Friedman and Malcolm Morgan for producing the Microsoft Word thesis template used to produce this document.

Thank you to Creative Commons Attribution 4.0 International License (CC BY) for letting authors keep the rights to their own works. 


\section{CONTENTS}

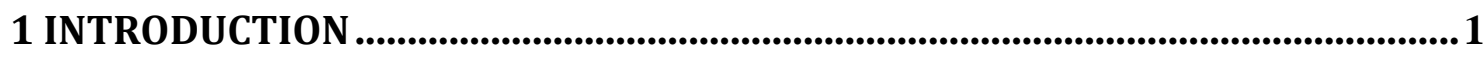

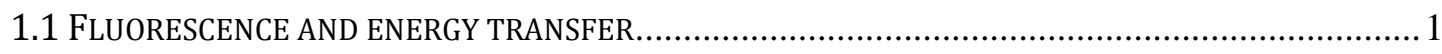

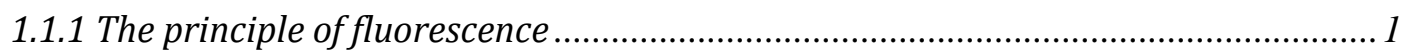

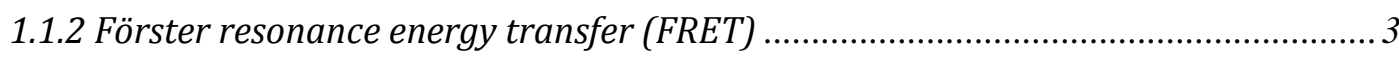

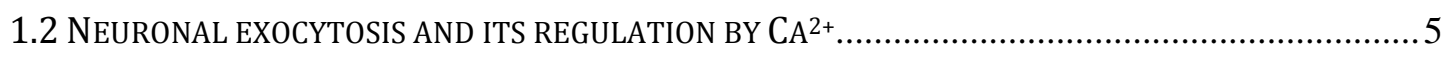

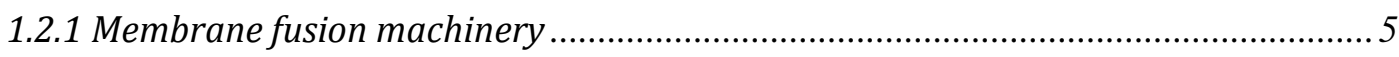

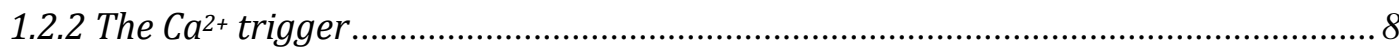

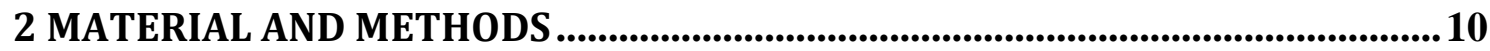

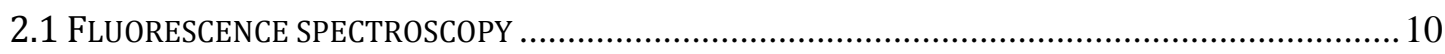

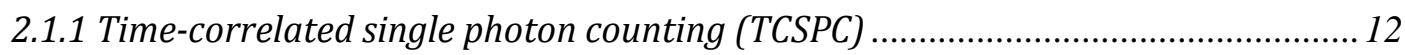

2.1.2 Fluorescence correlation spectroscopy (FCS) ...................................................... 15

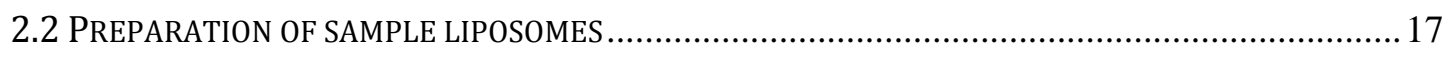

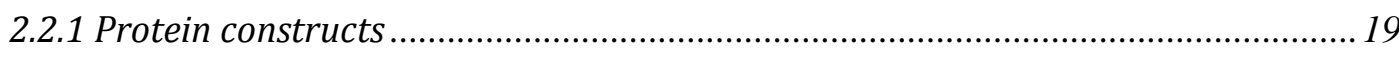

2.2.2 Synthesis and characterization of lipid-anchored DNA oligonucleotides................20

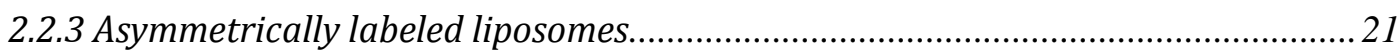

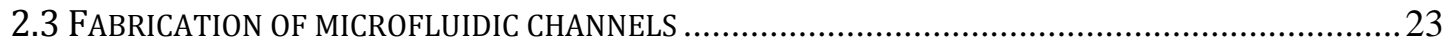

3 RESULTS AND DISCUSSION_......................................................................................... 25

3.1 CONTROL OF MEMBRANE GAPS BY SYNAPTOTAGMIN-CA ${ }^{2+}$ MEASURED WITH A NOVEL MEMBRANE

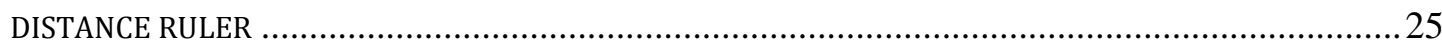

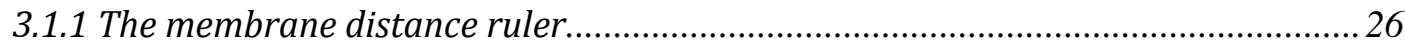

3.1.2 Synaptotagmin-1 controls the gap between two membranes.................................30

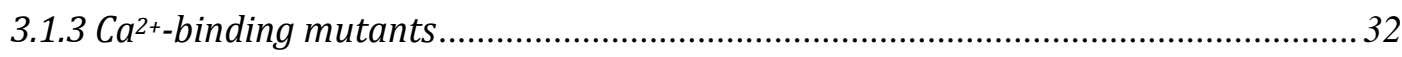

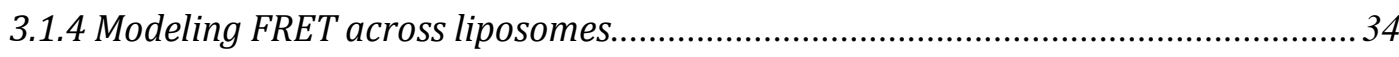

3.2 ASYMMETRICALLY LABELED LIPOSOMES AS A NEW TOOL TO STUDY MEMBRANE FUSION .............38

3.2.1 Differentiating two acceptor labels with the fluorescence lifetime ......................... 40

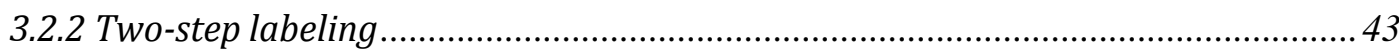

3.2.3 The rapid transition through hemifusion............................................................... 44 
4 OUTLOOK

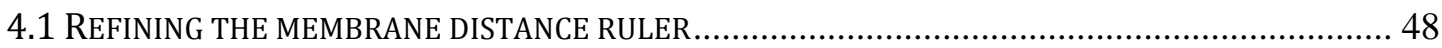

4.2 OPTIMAL DESIGN OF FRET FROM ONE DONOR TO TWO ACCEPTORS ..........................................49

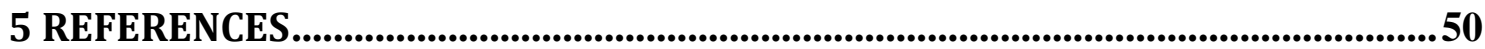

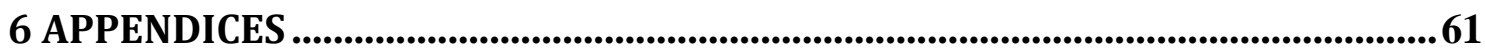




\section{LIST OF TABLES}

TABLE 2.1. SEQUENCES AND MS ANALYSIS OF THE TITLED OLIGONUCLEOTIDES. 20

TABLE 3.1. THE NUMBER OF ACCEPTOR LIPOSOMES BOUND TO EACH DONOR LIPOSOME DETERMINED FROM 2-COMPONENT FITTING OF THE AUTOCORRELATION CURVES............................................. 32

TABLE 3.2. RELATIVE AMPLITUDES AT 565 NM FROM TWO SETS OF MEASUREMENTS...........................41

TABLE 3.3. RISE TIME CONSTANTS AND ASSOCIATED AMPLITUDES FROM THE FITS TO FIGURE $3.15 \ldots 42$ 


\section{LIST OF FIGURES}

FIGURE 1.1. RELAXATION MECHANISM FOLLOWING THE ABSORPTION OF LIGHT. .................................. 2

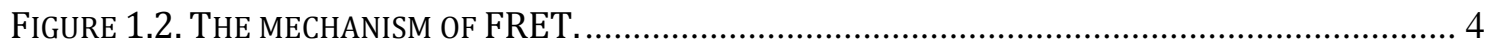

FIGURE 1.3. VESICLE TRAFFICKING CYCLE AT A PRESYNAPTIC TERMINAL.......................................... 5

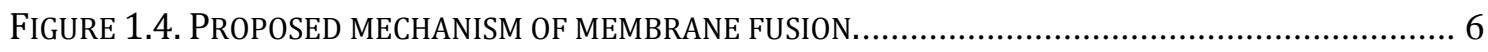

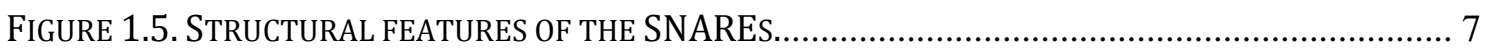

FIGURE 1.6. SCHEMATIC SKETCH OF THE SYNAPTOTAGMIN-1 DOMAIN STRUCTURE.............................. 8

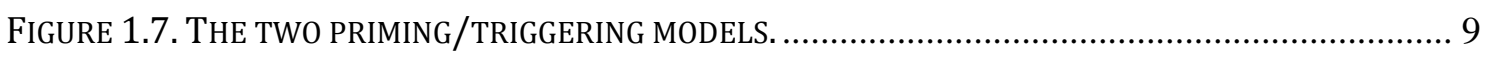

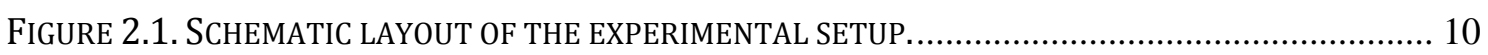

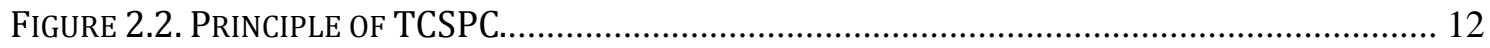

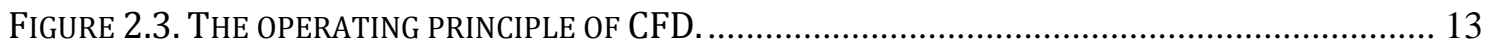

FIGURE 2.4. CONVOLUTION OF THE FLUORESCENCE DECAY WITH IRF............................................ 14

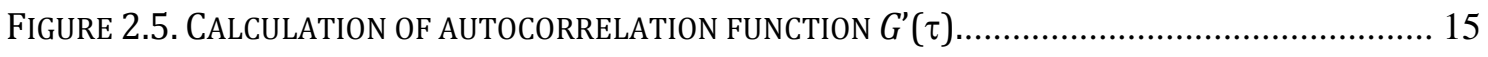

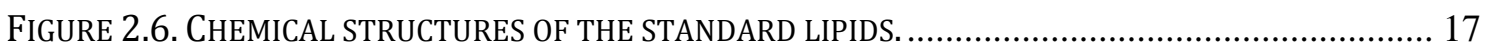

FigURE 2.7. CHEMICAL STRUCTURES OF FLUORESCENTLY LABELED LIPIDS. ....................................... 18

FIGURE 2.8. MECHANISM OF LIPOSOME FORMATION USING SIZE-EXCLUSION CHROMATOGRAPHY ......... 19

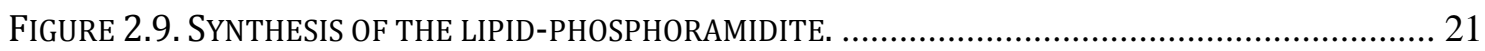

FIGURE 2.10. THE ONE-POT, TWO-STEP REACTION TO PRODUCE ASYMMETRICALLY LABELED LIPOSOMES. 22

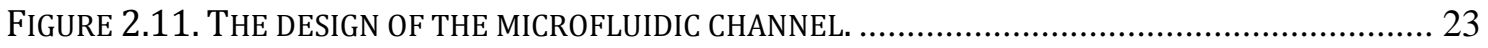

FIGURE 3.1. FRET FROM GREEN DONOR LIPOSOMES TO THE SURROUNDING RED ACCEPTOR LIPOSOMES. 26

FIGURE 3.2. CONVERGENCE OF THE FLUORESCENCE DECAY CURVES AT HIGHER EXCESS RATIOS. .......... 27

FIGURE 3.3. THE MEASURED FLUORESCENCE DECAY CURVES AND THE MEMBRANE DISTANCE RULER FOR CONVERTING FITTED $\tau_{\text {AMP }}$ INTO CLOSEST DISTANCES BETWEEN SMALL UNILAMELLAR LIPOSOMES. 28

FIGURE 3.4. SPECTRA OF THE FLUOROPHORES AND BAND PASS FILTERS. 29

FIGURE 3.5. INHIBITION OF DNA-LIPOSOMES HYBRIDIZATION VIA THE ADDITION OF A FREE SINGLE

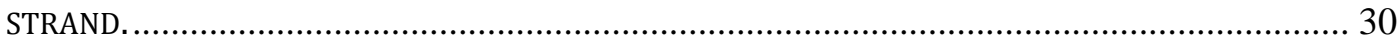


FIGURE 3.6. AUTOCORRELATION CURVES OF THE ACCEPTOR SIGNALS AND THE 2-COMPONENT FITTING.

FIGURE 3.7. MODEL OF FULL-LENGTH SYT-1 BINDING ACROSS THE SYNAPTIC VESICLE AND THE

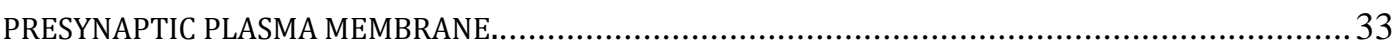

FIGURE 3.8. DISTRIBUTION OF DONOR (GREEN) AND ACCEPTOR (RED) LABELS IN THE MODELING........35

FIGURE 3.9. MODELING OF FRET FROM DONOR TO ACCEPTOR LIPOSOMES. ........................................36

FIGURE 3.10. INFLUENCE OF SOLVENT RELAXATION ON THE FLUORESCENCE DECAYS MEASURED AT

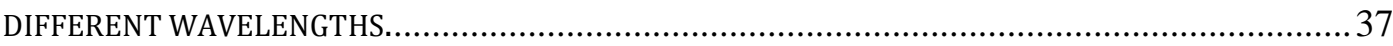

FIGURE 3.11. SPECTRA OF THE ONE DONOR-TWO ACCEPTOR SYSTEM. ............................................... 38

FIGURE 3.12. FLUORESCENCE DECAYS OF LIPOSOMES WITH VARIOUS LABELING PERCENTAGES,

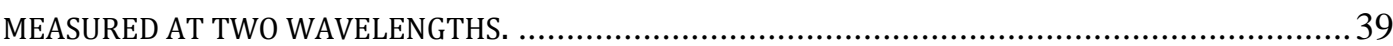

FIGURE 3.13. FLUORESCENCE DECAYS OF A SECOND SET OF MIXTURES TOWARDS LESS OREGON GREEN 488 .40

FIGURE 3.14. FLUORESCENCE DECAYS OF LIPOSOME MIXTURES MIMICKING THE PROGRESSION FROM HEMIFUSION TO FULL FUSION.

FigURE 3.15. THE DEVIATION OF ACCEPTOR SIGNALS AT EARLY TIMES VARIED WITH LABELING PERCENTAGES. 42

FIGURE 3.16. CHARACTERIZATION OF THE TWO REACTIONS WITH FCS. 43

FIGURE 3.17. SNARE-MEDIATED MEMBRANE FUSION OBSERVED IN A MICROFLUIDIC CHANNEL SHOWED FASTER KINETICS THAN IN A CUVETTE. .46 


\section{LIST OF ABBREVIATIONS AND ACRONYMS}

\begin{tabular}{|c|c|}
\hline APD & avalanche photodiodes \\
\hline bp & base pairs \\
\hline DPPE & 1,2-dipalmitoyl-sn-glycero-3-phosphoethanolamine \\
\hline DPPTE & 1,2-dipalmitoyl-sn-glycero-3-phosphothioethanol \\
\hline EGTA & ethylene glycol tetraacetic acid \\
\hline ESI-MS & electrospray ionization mass spectrometry \\
\hline FCS & fluorescence correlation spectroscopy \\
\hline FRET & Förster resonance energy transfer \\
\hline FWHM & full-width half-maximum \\
\hline HEPES & 4-(2-hydroxyethyl)-1-piperazineethanesulfonic acid \\
\hline IRF & instrument response function \\
\hline KKKK & poly-lysine \\
\hline Laurdan & 2-dimethylamino-6-lauroylnaphthalene \\
\hline MB & Marina Blue DPPE \\
\hline NBD & $N$-(7-nitrobenz-2-oxa-1,3-diazol-4-yl)-DPPE \\
\hline OG & Oregon Green 488 \\
\hline PIP2 & phosphatidylinositol-4,5-bisphosphate \\
\hline Prodan & 6-propionyl-2-dimethylaminonaphthalene \\
\hline PS & DOPS, 1,2-dioleoyl-sn-glycero-3-phospho-L-serine \\
\hline RP-HPLC & reversed-phase high-performance liquid chromatography \\
\hline SNARE & soluble $N$-ethylmaleimide-sensitive factor attachment protein receptor \\
\hline syt-1 & synaptotagmin-1 \\
\hline$\tau_{\mathrm{amp}}$ & amplitude-weighted lifetime \\
\hline TCSPC & time-correlated single photon counting \\
\hline
\end{tabular}




\section{LIST OF APPENDICES}

CURRICULUM VITAE ... 62

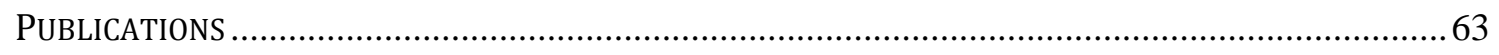




\section{INTRODUCTION}

\subsection{Fluorescence and energy transfer}

\subsubsection{The principle of fluorescence}

For a conjugated organic molecule, which contains a number of alternating single and double bonds or multiple aromatic rings, the energy difference between the highest of many closely spaced $\pi$-bonding or non-bonding orbitals (highest occupied molecular orbital, HOMO) and the lowest $\pi^{*}$-antibonding orbital (lowest unoccupied molecular orbital, LUMO) very often matches the energy of a photon in the visible range. Absorption of a photon by the molecule promotes an electron to an unoccupied orbital such that the molecule is in an electronic excited state $\left(S_{n}, n \geq 1\right)$. Following excitation, a molecule in solution undergoes ultrafast relaxation (typically $<10 \mathrm{ps}$ ) with near unity efficiency to the lowest-lying excited state $\left(\mathrm{S}_{1}\right)$ with minimum vibrational energy $(v=0)$. The relaxation processes include (i) redistributing the energy to vibrational modes with lower frequencies (intramolecular vibrational redistribution, IVR), (ii) dissipating excess vibrational energy via collision with solvent molecules (vibrational relaxation, VR) and (iii) crossing to the potential energy surface (PES) of a lower electronic state (internal conversion, IC). All of which are driven by the tendency to populate as many states as possible at any defined energy interval.

After reaching $S_{1}(v=0)$, the ensuing deactivation processes are usually two to three orders of magnitude slower (>1 ns), a condition which is sometimes referred to as the "Kasha's rule". The molecule will return to the electronic ground state $S_{0}$ (illustrated in Figure 1.1) via (i) emitting a photon, i.e., fluorescence, at a rate of $k_{\mathrm{f}}$ or (ii) deactivating through non-radiative pathways $\left(k_{\mathrm{nr}}\right)$. Non-radiative pathways grouped together here may be internal conversion from $S_{1}$ to $S_{0}$ or transitions through lower-lying triplet excited states $\left(T_{m}, m \geq 1\right)$, in which, as 
opposed to singlet states $S_{n}$, the unpaired electrons may assume the same spin. Internal conversion from $S_{1}$ to $S_{0}$ is significantly slower than that from higher excited states to $S_{1}$, due to the fact that excited states are much more closely spaced in energy compared to the large energy gap between $S_{1}$ to $S_{0}$. For organic molecules, triplet excited states normally do not produce observable emission and are thus regarded as dark states.

The return of a molecule from $S_{1}$ to $S_{0}$ follows $1^{\text {st }}$ order kinetics:

$$
\begin{gathered}
\frac{d\left[\mathrm{~S}_{1}(t)\right]}{d t}=-\left(k_{\mathrm{f}}+k_{\mathrm{nr}}\right)\left[\mathrm{S}_{1}(t)\right] \\
{\left[\mathrm{S}_{1}(t)\right]=\left[\mathrm{S}_{1}(0)\right] e^{-\left(k_{\mathrm{f}}+k_{\mathrm{nr}}\right) t}=\left[\mathrm{S}_{1}(0)\right] e^{-t / \tau_{\mathrm{obs}}}}
\end{gathered}
$$

where $\left[S_{1}(0)\right]$ stands for the initial concentration of molecules in the $S_{1}$ state generated by excitation. The fluorescence intensity is proportional to the number of molecules remaining in $\mathrm{S}_{1}$ and therefore decays at the same overall rate, whose inverse is commonly expressed as the "observed lifetime $\left(\tau_{\mathrm{obs}}\right)$ ". Fluorescence allows for highly sensitive detection, a textbook analogy being having a few candles lit in an otherwise dark stadium. Consequently, fluorescent artificial molecules or protein segments are powerful tools in the study of complex biological structures or dynamics.
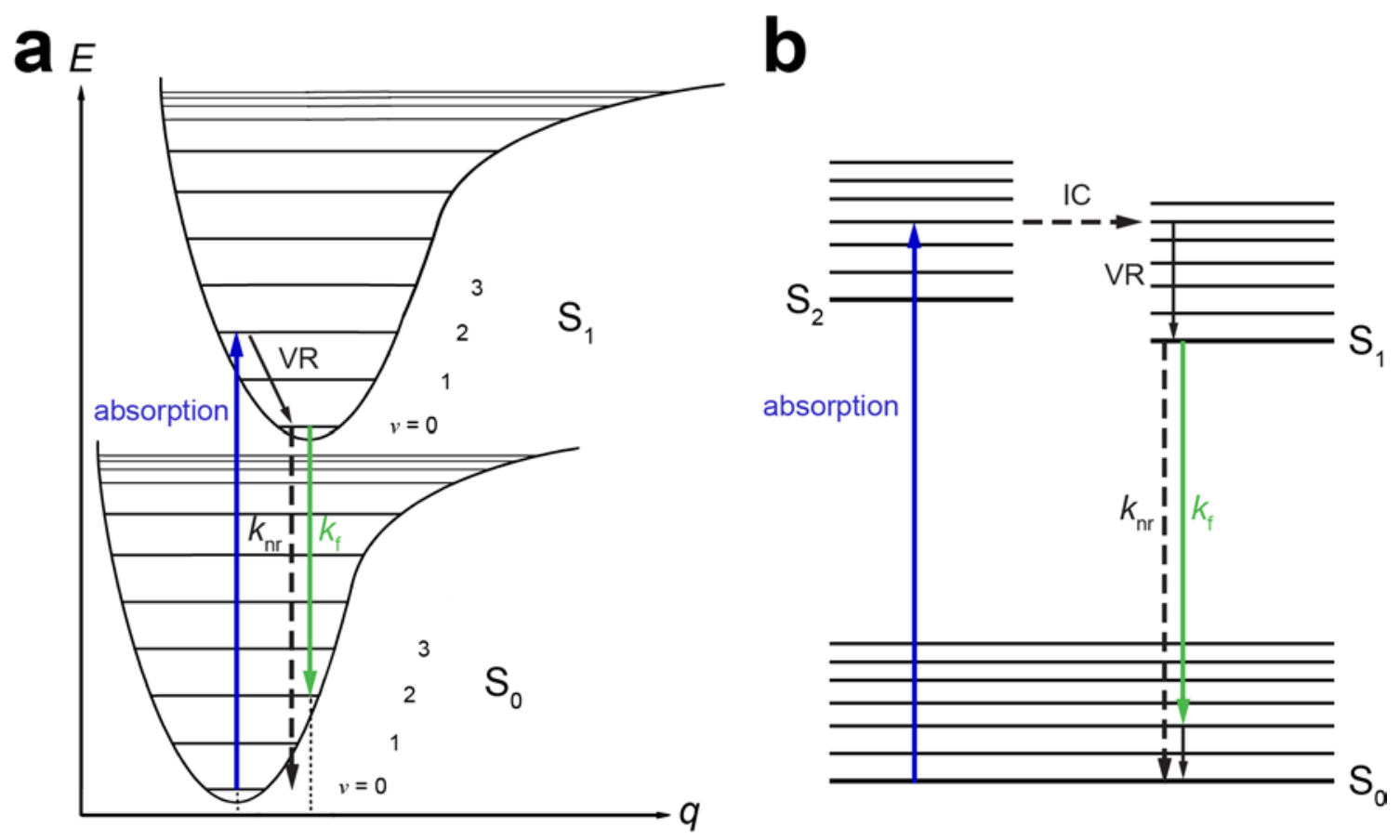

Figure 1.1. Relaxation mechanism following the absorption of light. (a) Plotted on potential energy surfaces of $S_{1}$ and $S_{0}$. The $y$-axis is energy and the $x$-axis is a representative vibrational mode. (b) A simplified Jablonski diagram showing excitation to the $\mathrm{S}_{2}$ state. 
In Figure 1.1a, it can also be seen that the energy of an emitted fluorescence photon is lower than that of the originally absorbed one, so that the emission spectrum is red-shifted (i.e., Stokes shift) from the absorption. ${ }^{2}$ This arises from the relatively slow nuclear motions with respect to the almost instantaneous electronic transitions ( $<1 \mathrm{fs}$ ). Accordingly, the electronic transitions are always drawn vertically, during which the nuclear positions remain fixed. At electronic excited states, because of the occupation of an antibonding orbital, the equilibrated bond lengths are usually longer, resulting in displacement of the potential energy surfaces along certain vibrational coordinates. Since fluorescence originates from $\mathrm{S}_{1}(v=0)$, the downward vertical transition becomes less energetic. As a matter of fact, fluorescence from higher singlet excited states to $\mathrm{S}_{0}$ can also occur, but is often not competitive with internal conversion to $\mathrm{S}_{1}$ and is only observable at very early delay times in femtosecond spectroscopy (for example, in reference 3).

\subsubsection{Förster resonance energy transfer (FRET)}

When the emission spectrum of a molecule overlaps with the absorption of another, energy transfer from the former (donor) to the latter (acceptor) may occur without first emitting a photon, which is named Förster resonance energy transfer (FRET). ${ }^{4}$ The energy transfer rate, $k_{\mathrm{ET}}$, is given by:

$$
k_{\mathrm{ET}}=\frac{Q_{D} \kappa^{2}}{\tau_{D 0} r^{6}} \cdot \frac{9000(\ln 10)}{128 \pi^{5} N_{A} n^{4}} \cdot \int_{0}^{\infty} F_{D}(\lambda) \varepsilon_{A}(\lambda) \lambda^{4} d \lambda
$$

where $Q_{\mathrm{D}}$ and $\tau_{\mathrm{D} 0}$ are the fluorescence quantum yield $\left(k_{\mathrm{f}} /\left(k_{\mathrm{f}}+k_{\mathrm{nr}}\right)\right)$ and observed lifetime of the donor in the absence of acceptor, respectively. $N_{\mathrm{A}}$ is the Avogadro's number, and $n$ is the refractive index. $F_{\mathrm{D}}(\lambda)$ implies the donor fluorescence intensity at each wavelength, with the total area under the curve normalized to unity; $\varepsilon_{\mathrm{A}}(\lambda)$ denotes the acceptor absorption extinction coefficient (in units of $\mathrm{M}^{-1} \mathrm{~cm}^{-1}$ ). Finally, $r$ is the distance between the donor and acceptor, and $\kappa^{2}$ describes the relative orientation of the donor and acceptor transition dipoles in space (arrows in Figure 1.2a):

$$
\kappa^{2}=\left(\cos \theta_{T}-3 \cos \theta_{D} \cos \theta_{A}\right)^{2}
$$

The $1 / r^{6}$ dependence stems from the oscillating dipole-like nature of the electronic transitions. The potential between two dipoles gives an $1 / r^{3}$ dependence, and Fermi's golden rule states that the transition rate is proportional to the square of the interaction, hence yielding $1 / r^{6}$. For convenience, Equation 1.3 is regularly expressed as:

$$
k_{\mathrm{ET}}(r)=\frac{1}{\tau_{D 0}} \cdot\left(\frac{R_{0}}{r}\right)^{6}
$$

with the Förster distance $R_{0}$ specifying the distance (when $r=R_{0}$ ) at which $k_{\mathrm{ET}}$ equals the intrinsic decay rate of the donor $\left(\tau_{\mathrm{D} 0}{ }^{-1}\right)$ : 


$$
R_{0}=0.0211\left[\kappa^{2} n^{-4} Q_{D} \int_{0}^{\infty} F_{D}(\lambda) \varepsilon_{A}(\lambda) \lambda^{4} d \lambda\right]^{\frac{1}{6}}
$$

if wavelength in the integral is expressed in $\mathrm{nm}$.

\section{a}

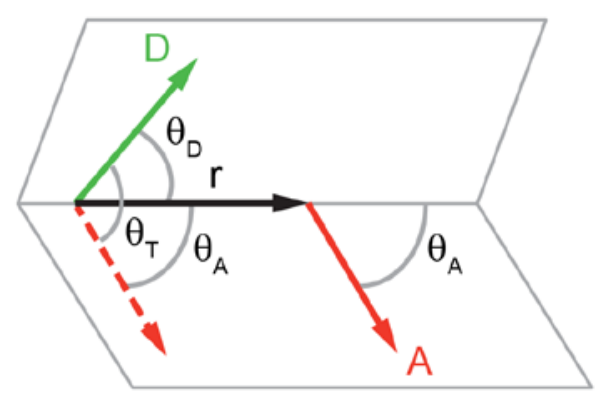

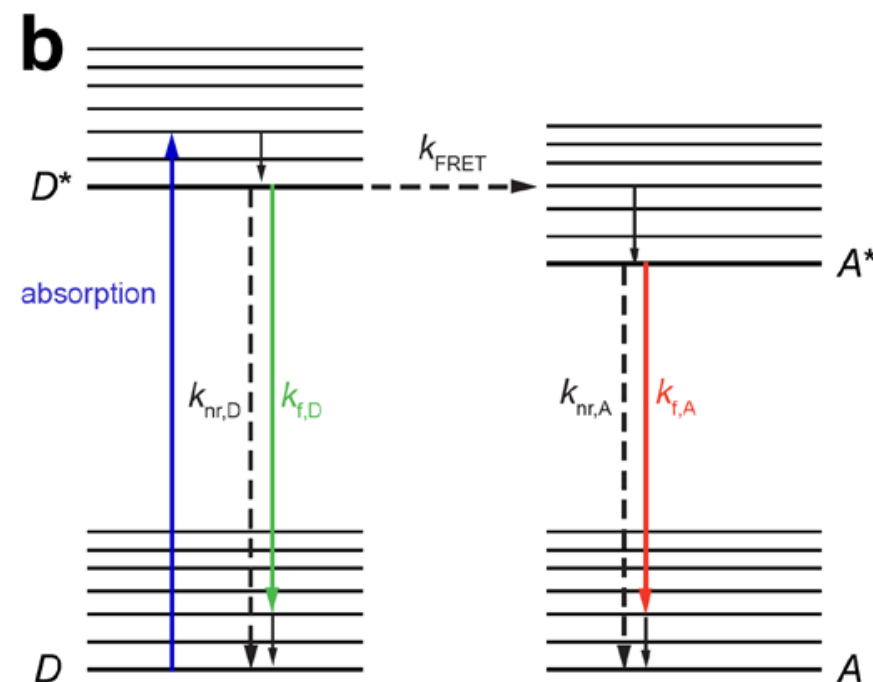

Figure 1.2. The mechanism of FRET. (a) The donor emission dipole and acceptor absorption dipole, with a distance of $r$ and three angles to describe their relative orientation in space. (b) Jablonski diagram including FRET.

FRET adds an addition deactivation pathway to the donor $\mathrm{S}_{1}$ state, now relabeled as $D^{*}$ (Figure 1.2b), so its concentration time-dependence becomes:

$$
\left[D^{*}(t)\right]=\left[D^{*}(0)\right] e^{-\left(k_{\mathrm{f}, \mathrm{D}}+k_{\mathrm{nr}, \mathrm{D}}+k_{\mathrm{ET}}\right) t}=\left[D^{*}(0)\right] e^{-t / \tau_{\mathrm{D}}}
$$

and the observed lifetime $\tau_{\mathrm{D}}$ is shorter than the intrinsic lifetime $\tau_{\mathrm{D} 0}$. On the other hand, population of the acceptor excited state $\left(A^{*}\right)$ via FRET follows a precursor-successor relationship:

$$
\begin{gathered}
\frac{d\left[A^{*}(t)\right]}{d t}=\left(k_{\mathrm{f}, \mathrm{D}}+k_{\mathrm{nr}, \mathrm{D}}+k_{\mathrm{ET}}\right)\left[D^{*}(t)\right]-\left(k_{\mathrm{f}, \mathrm{A}}+k_{\mathrm{nr}, \mathrm{A}}\right)\left[A^{*}(t)\right]=k_{1}\left[D^{*}(t)\right]-k_{2}\left[A^{*}(t)\right] \\
{\left[A^{*}(t)\right]=\frac{\left[D^{*}(0)\right] k_{1}}{k_{1}-k_{2}}\left(-e^{-k_{1} t}+e^{-k_{2} t}\right)=\frac{\left[D^{*}(0)\right] k_{1}}{k_{1}-k_{2}}\left(-e^{-t / \tau_{\mathrm{D}}}+e^{-t / \tau_{\mathrm{A} 0}}\right)}
\end{gathered}
$$

assuming that direct excitation of the acceptor is negligible. In typical experimental designs, the distance $r$ between the donor and acceptor is sufficiently short such that $k_{1}>k_{2}$, and then $\tau_{\mathrm{D}}$ becomes a rise time constant, whereas the decay time constant of the acceptor $\left(\tau_{\mathrm{A}}\right)$ remains the same as that when it is directly excited $\left(\tau_{\mathrm{A} 0}\right)$. 


\subsection{Neuronal exocytosis and its regulation by $\mathrm{Ca}^{2+}$}

\subsubsection{Membrane fusion machinery}

Synapses are where nerve cells (neurons) are connected, with a gap (synaptic cleft) of a few tens of nanometers in between. Messages are delivered from the presynaptic neuron to the postsynaptic neuron via release of neurotransmitters (e.g., glutamate) from the former into the cleft (exocytosis, Figure 1.3) ${ }^{5}$ to be captured by receptors residing on the latter. The neurotransmitters are originally packaged in synaptic vesicles $\sim 40 \mathrm{~nm}$ in diameter. To release them, the vesicles have to be fused with the presynaptic plasma membrane. Both the vesicle and presynaptic membranes are bilayers consist primarily of phospholipids, each featuring a hydrophilic head group and two hydrophobic tails. Fusion requires first local deformation of the bilayers (Figure 1.4$)^{6,7}$ and is subject to high lipid composition-dependent energy barriers ( 80 $\left.k_{\mathrm{B}} \mathrm{T}\right)^{8}$

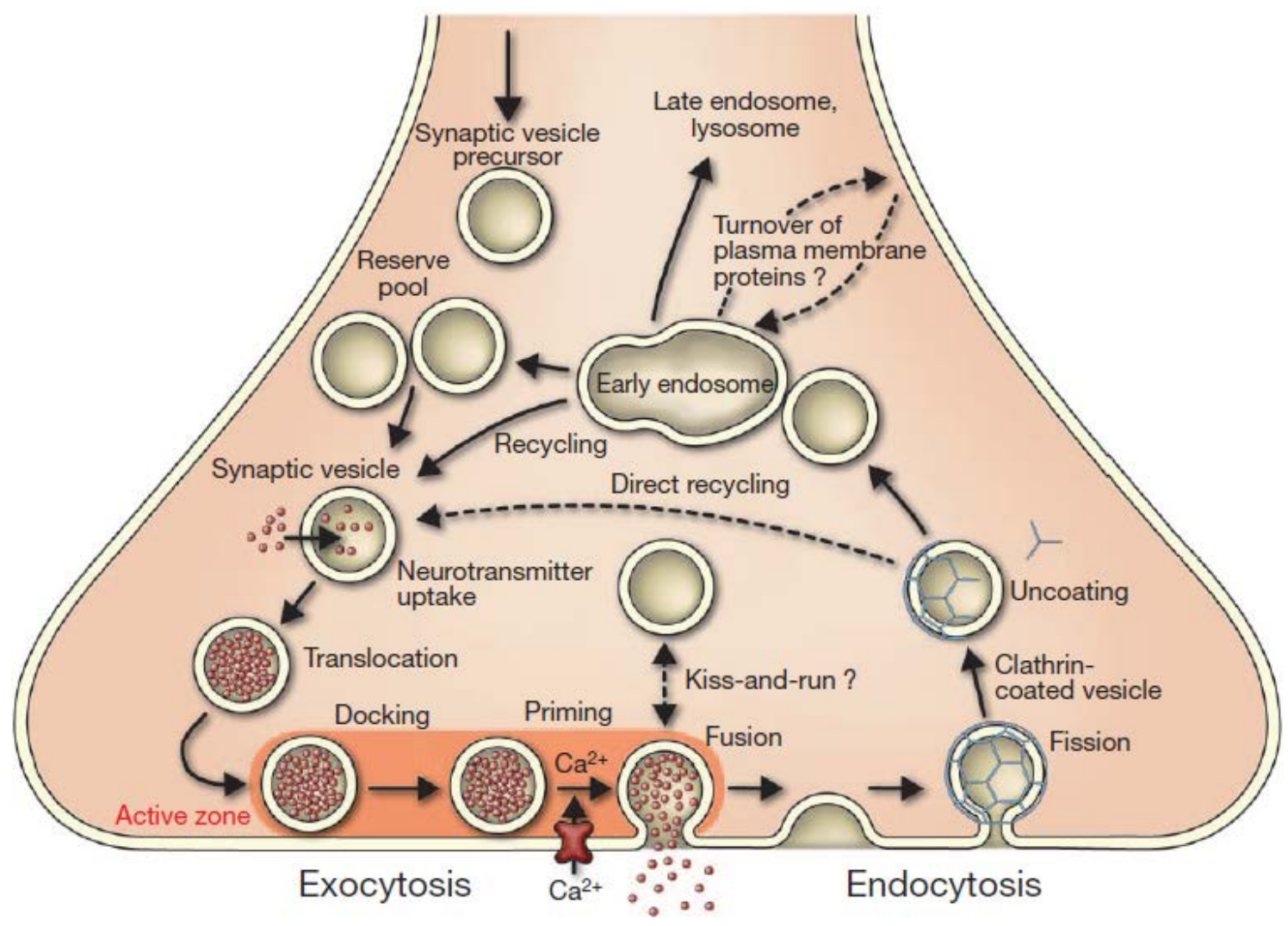

Figure 1.3. Vesicle trafficking cycle at a presynaptic terminal. This study focuses on the docking, priming and fusion processes in the highlighted active zone. Adapted by permission from Macmillan Publishers Ltd: Nature (ref. 5), copyright (2012). 
In vivo, the energy barrier is overcome by a set of protein membrane fusion machinery, soluble $N$-ethylmaleimide-sensitive factor attachment protein receptor (SNARE, Figure 1.4b). SNARE proteins are essential for the fusion of trafficking vesicles with target membranes, conserved from yeast to humans. ${ }^{9}$ The SNARE machinery studied in this work comes from rat neurons (Rattus norvegicus) and comprises three members: synaptobrevin-2 (also often referred to as vesicle-associated membrane protein 2, VAMP-2), which is the most abundant protein located on synaptic vesicles (therefore called vesicle SNARE, or $v$-SNARE), ${ }^{10}$ (ii) syntaxin-1A and (iii) SNAP-25A, both at the presynaptic membrane (target SNAREs, or t-SNAREs). Synaprobrevin-2 and syntaxin-1A each possesses a C-terminal transmembrane domain (TMD), and SNAP-25A is anchored to the membrane through palmitoylation (fatty acid $\left.\mathrm{CH}_{3}\left(\mathrm{CH}_{2}\right)_{14} \mathrm{COOH}\right)$ to any of its four cysteine residues (Figure 1.5a).

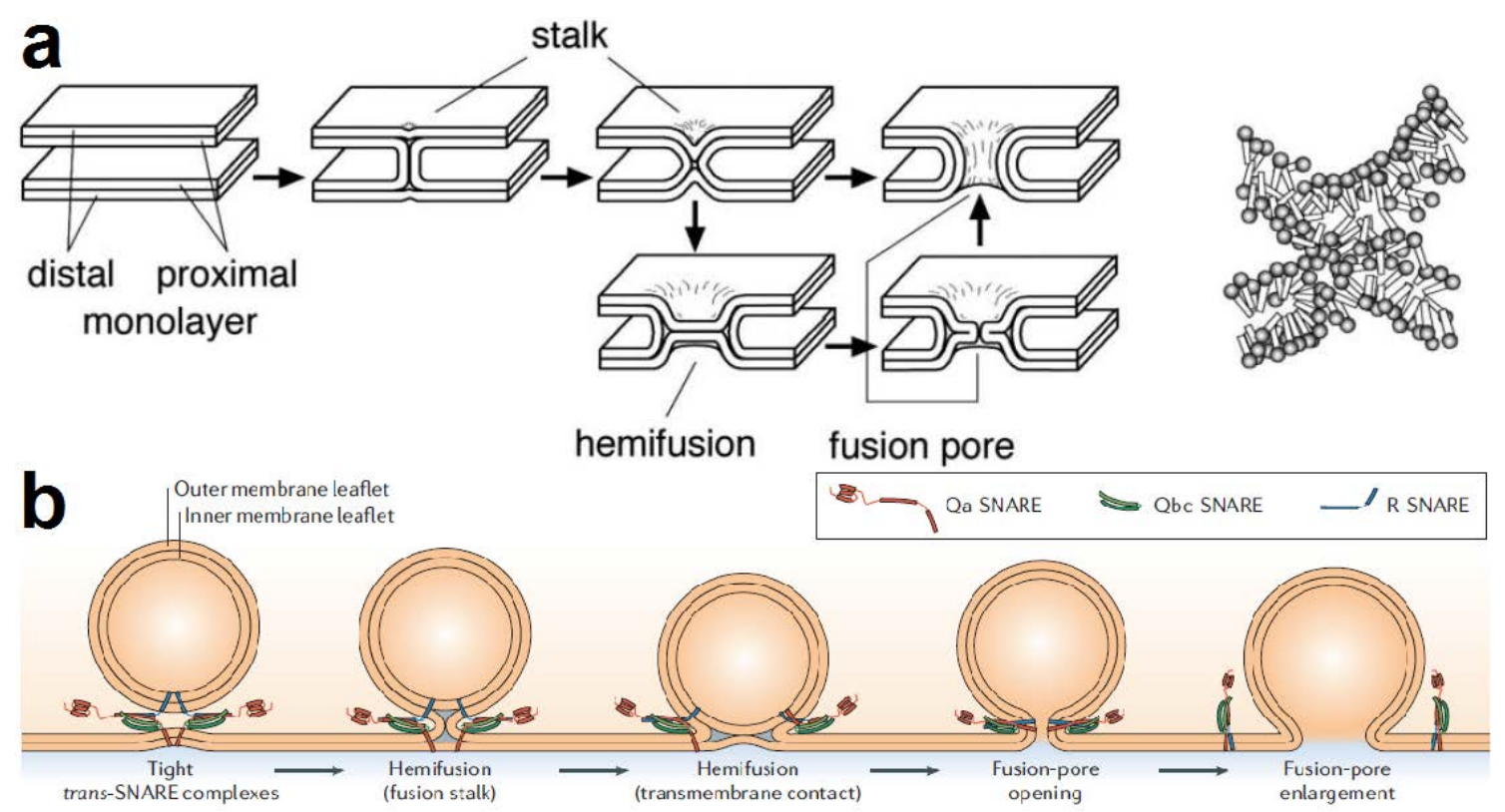

Figure 1.4. Proposed mechanism of membrane fusion. With the aid of advanced molecular dynamics simulations. (a) Sketches showing possible transition states and the scrambling of lipids. (b) Fusion mediated by the SNARE proteins. Adapted by permissions from Elsevier \& Macmillan Publishers Ltd: Cell (ref. 6) and Nat. Rev. Mol. Cell Biol. (ref. 11), respectively, copyrights (2003 \& 2006).

SNAREs facilitate membrane fusion by forming a coil coiled four-helix bundle with their evolutionarily conserved SNARE motifs consisting of 60-70 amino acids (Figure 1.5b), starting from the membrane distal N-terminals. Synaprobrevin-2 and syntaxin-1A each provides one helix, while SNAP-25A provides two. The "zippering” progresses towards the C-terminal 
ends after the initial contact and eventually exert forces onto and in turn deforms the membranes. At the very well conserved central ionic layer of the bundle, synatobrevin- 2 contributes an arginine residue (arg, R), so it is re-classified structurally as an R-SNARE more recently. On the other hand, syntaxin-1A and SNAP-25A contribute glutamines (gln, Q), and are re-classified as Q-SNAREs. ${ }^{9}$ Full zippering of a trans-SNARE complex (spanning two membranes) releases $\sim 35 k_{\mathrm{B}} \mathrm{T},{ }^{12}$ so several complexes working in synergy suffices to overcome the energy barrier for fusion.
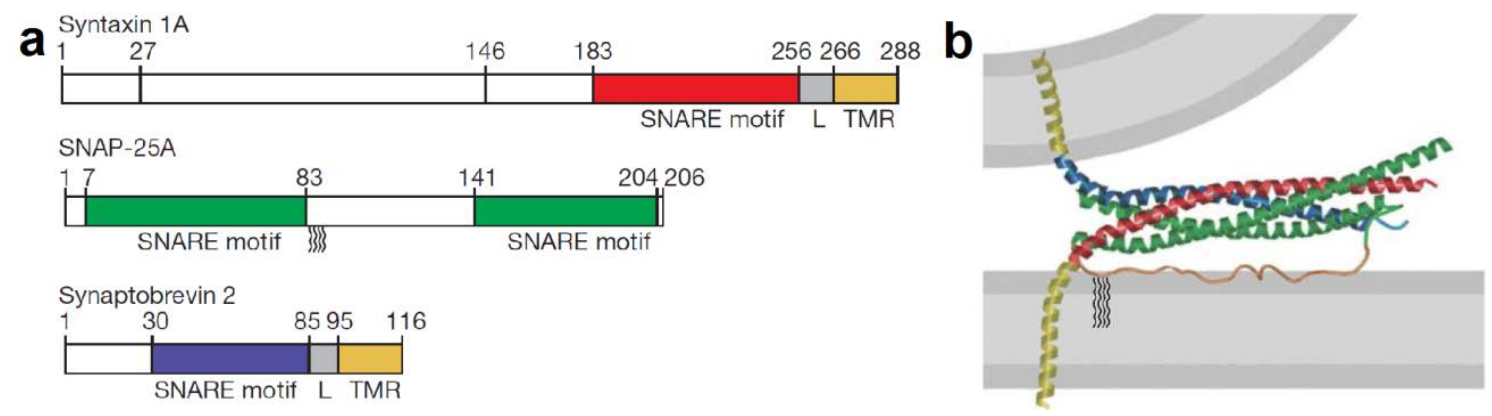

Figure 1.5. Structural features of the SNAREs. (a) Schematic depiction of domain structures. (b) A model of the trans-SNARE complex, with the four-helix bundle structurally characterized by crystallography. Adapted by permissions from Macmillan Publishers Ltd: Nature (refs. 13 and 14), copyrights (2009 \& 1998).

Understanding of the SNAREs has been facilitated by isolation and reconstitution of the proteins into artificial liposomes, ${ }^{15}$ using FRET to detect the resulting membrane fusion. The $v$ and $t$-SNAREs may be reconstituted into liposomes labeled with FRET donors and acceptors, respectively, so that fusion corresponds to the increase of FRET. ${ }^{16}$ Alternatively, donors and acceptors can be incorporated in the same type of liposomes, and fusion with unlabeled liposomes leads to a decrease of FRET. ${ }^{17}$

Besides the abovementioned bulk liposome fusion assays, single-molecule fluorescence techniques ${ }^{18}$ have been applied to study fusion of liposomes to surface-tethered liposomes, ${ }^{19-21}$ to giant unilamellar liposomes $>100 \mu \mathrm{m}$ in diameter ${ }^{22}$ or to supported planar bilayers. ${ }^{23,24}$ However, despite the extensive research, still under debate are the questions of how many SNARE complexes are needed exactly for a single fusion event, ${ }^{25,26}$ and whether the hemifusion state (Figure 1.4) where only the outer leaflets of the bilayers are merged constitutes a unstable transition state, a stable intermediate or a dead-end product. ${ }^{19}$ 


\subsubsection{The $\mathrm{Ca}^{2+}$ trigger}

Already highlighted in Figure 1.3, synaptic vesicles are first docked and primed to the presynaptic plasma membrane, and a millisecond $\mathrm{Ca}^{2+}$ influx through voltage-gated $\mathrm{Ca}^{2+}$ channels triggers SNARE-mediated membrane fusion. ${ }^{27,28}$ The $\mathrm{Ca}^{2+}$ channels are sensitive to decreases of net intracellular negative charges (depolarization of membrane potential), which are initiated, for instance, by photo-induced isomerization and dissociation in the formation of vision $^{29}$ or pressure-induced mechanical bending in hearing. ${ }^{30}$

The $\mathrm{Ca}^{2+}$ influx, in turn, is sensed by the synaptic vesicle protein, synaptotagmin-1 (syt1). ${ }^{31,32}$ Syt-1 bears two $\mathrm{Ca}^{2+}$-binding $\mathrm{C} 2$ domains (C2A and C2B, Figure 1.6) which, after coordinating to multiple $\mathrm{Ca}^{2+}$ ions, bind to membranes containing negatively charged phospholipids, completing the $\mathrm{Ca}^{2+}$-coordination sites. ${ }^{33-35}$ Moreover, a patch of four lysine residues (KKKK, 324-327) located on the side of C2B interacts specifically with the polyanionic phosphatidylinositol-4,5-bisphosphate (PIP2) in a $\mathrm{Ca}^{2+}$-independent manner. ${ }^{36}$ However, in spite of extensive research using either full-length syt-1 (including the transmembrane domain, TMD) or the truncated soluble C2AB fragment, ${ }^{37-39}$ how syt-1 functions at the molecular level as a $\mathrm{Ca}^{2+}$-trigger and how it interacts with the SNAREs remained unsettled.

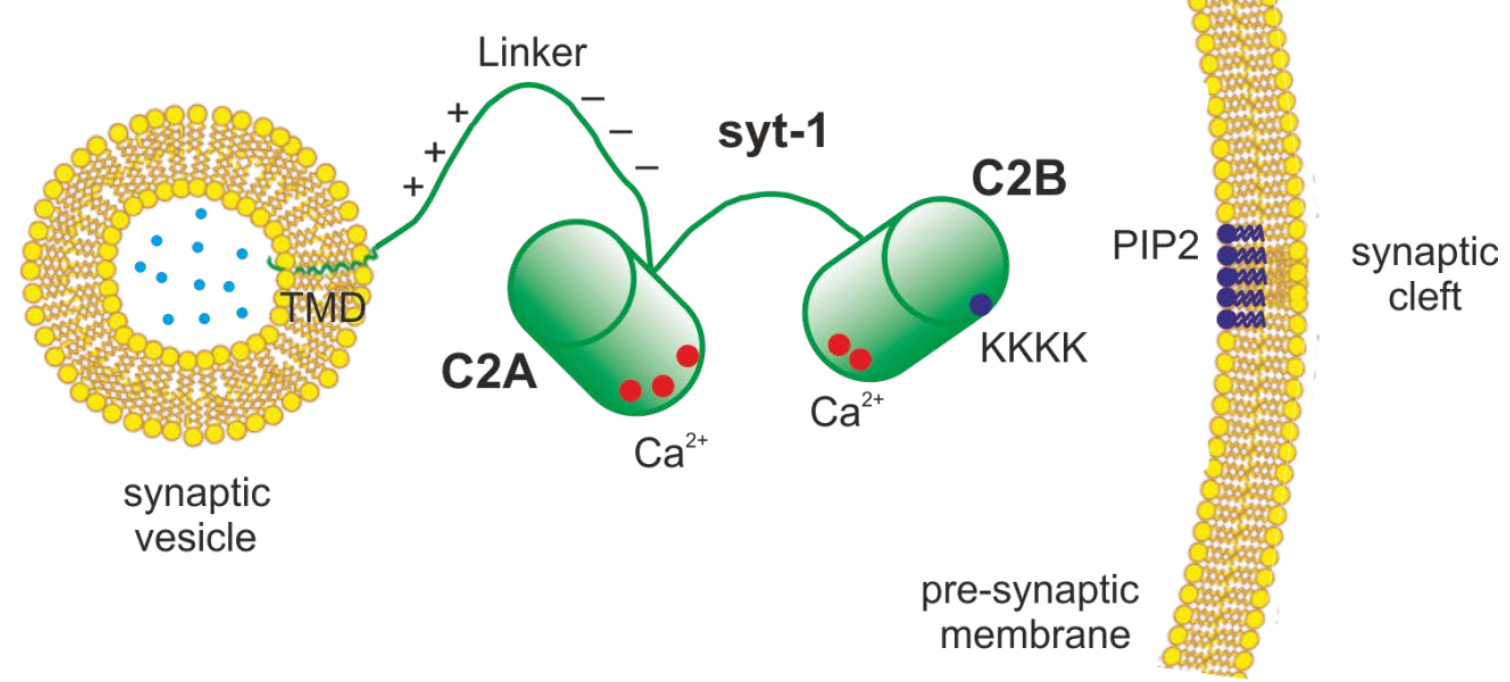

Figure 1.6. Schematic sketch of the synaptotagmin-1 domain structure. Red dots signify $\mathrm{Ca}^{2+}$ binding sites, whereas the dark blue dot denotes the poly-lysine patch (KKKK) of the $\mathrm{C} 2 \mathrm{~B}$ domain. Plus and minus signs indicate clusters of positive and negative charges on the linker. The vesicle is not drawn to scale. Courtesy of Prof. Dr. Peter Jomo Walla. 
Models describing the molecular activation of the fusion reaction by synaptotagmin fall into two groups (summarized in Figure 1.7). ${ }^{5}$ In the first scenario, the SNARE complexes are arrested at a partially zippered state during the priming process (Priming I). Syt-1 may either serve as a fusion clamp that is released on $\mathrm{Ca}^{2+}$ triggering, ${ }^{40}$ or alternatively, it may displace the inhibitory protein complexin from the SNARE complexes $^{41}$ (Triggering I). In the second scenario, the SNAREs do not assemble before arrival of $\mathrm{Ca}^{2+}$ signals, although syt-1 may already be in contact with the plasma membrane via the KKKK-PIP2 electrostatic interaction (Priming II). ${ }^{36}$ Following $\mathrm{Ca}^{2+}$ triggering, the SNAREs rapidly progress through zippering and fusion (Triggering II). ${ }^{42}$

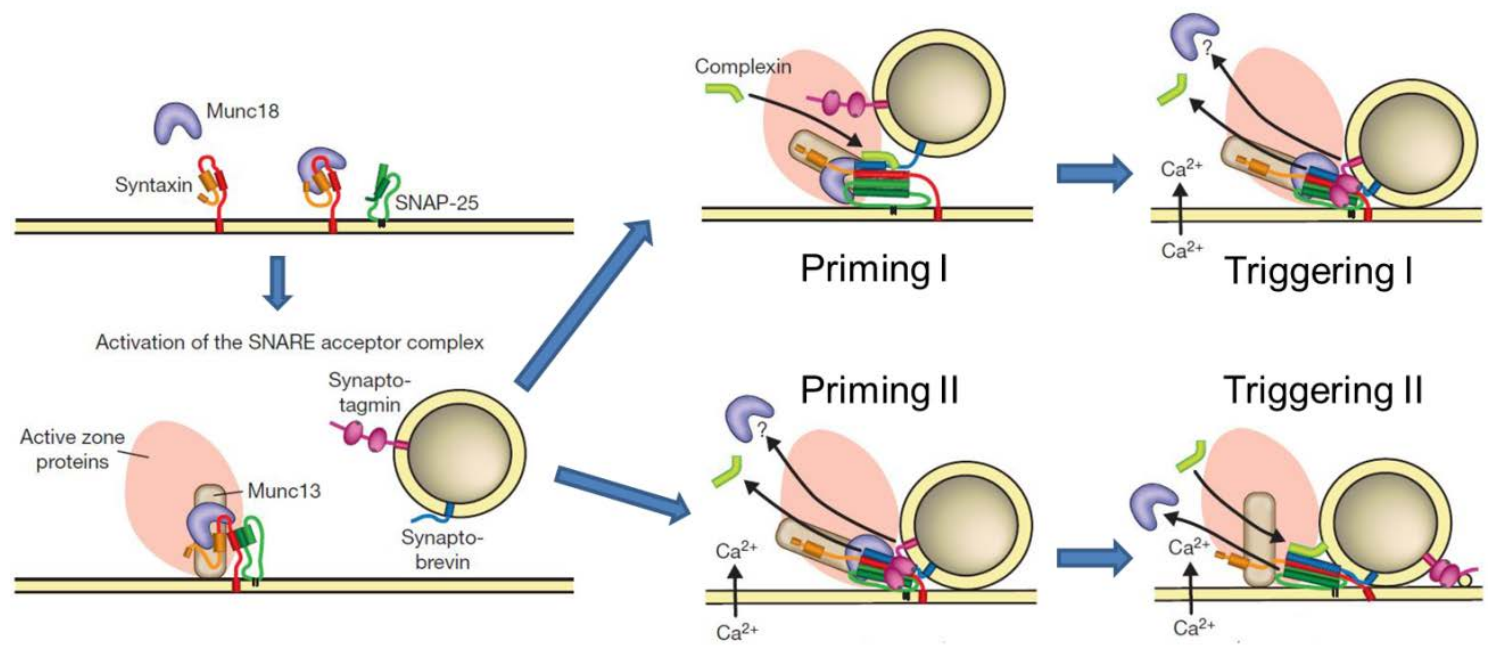

Figure 1.7. The two priming/triggering models. Also shown are Munc18, which binds to syntaxin-1A, and Munc13, which is thought to promote the formation of $t$-SNARE acceptor complexes (syntaxin-1A together with SNAP-25A). Adapted by permission from Macmillan Publishers Ltd: Nature (ref. 5), copyright (2012).

In line with the latter scenario, recently, it has been proposed that syt-1 triggering is based on $\mathrm{Ca}^{2+}$-dependent regulation of the gap between the vesicle and presynaptic membranes. ${ }^{43}$ In the absence of $\mathrm{Ca}^{2+}$, syt-1 connects membranes via (i) its own transmembrane domain and (ii) binding to PIP2 via the KKKK polybasic stretch. Assuming that the 61-residue linker between $\mathrm{C} 2 \mathrm{~A}$ and the transmembrane domain ${ }^{44}$ is unstructured and stretched, a maximal distance of $\sim 28$ $\mathrm{nm}$ is feasible. On the basis of non-quantitative FRET assays and molecular dynamics simulations, it was suggested that addition of $\mathrm{Ca}^{2+}$ might reduce the distance to a range of $\sim 2$ $7.5 \mathrm{~nm}$. Such distance shortening might operate upstream of the SNARE complex formation, which requires a membrane distance below $\sim 8 \mathrm{~nm} .{ }^{12}$ However, experimental support for this model was elusive, primarily because of the lack of experimental evidence for distance variations at the appropriate length scale. 


\section{MATERIAL AND METHODS}

\subsection{Fluorescence spectroscopy}

The measurements in this work, except for steady-state emission and excitation spectra or bulk liposome fusion assays, were all performed on a fluorescence microscope based on an Olympus IX71 inverted microscope body, an UPlanSApo 60×/1.20 W water immersion objective (Olympus) and two avalanche photodiodes (SPCM-CD 2969, PerkinElmer). The layout of the setup is depicted in Figure 2.1.

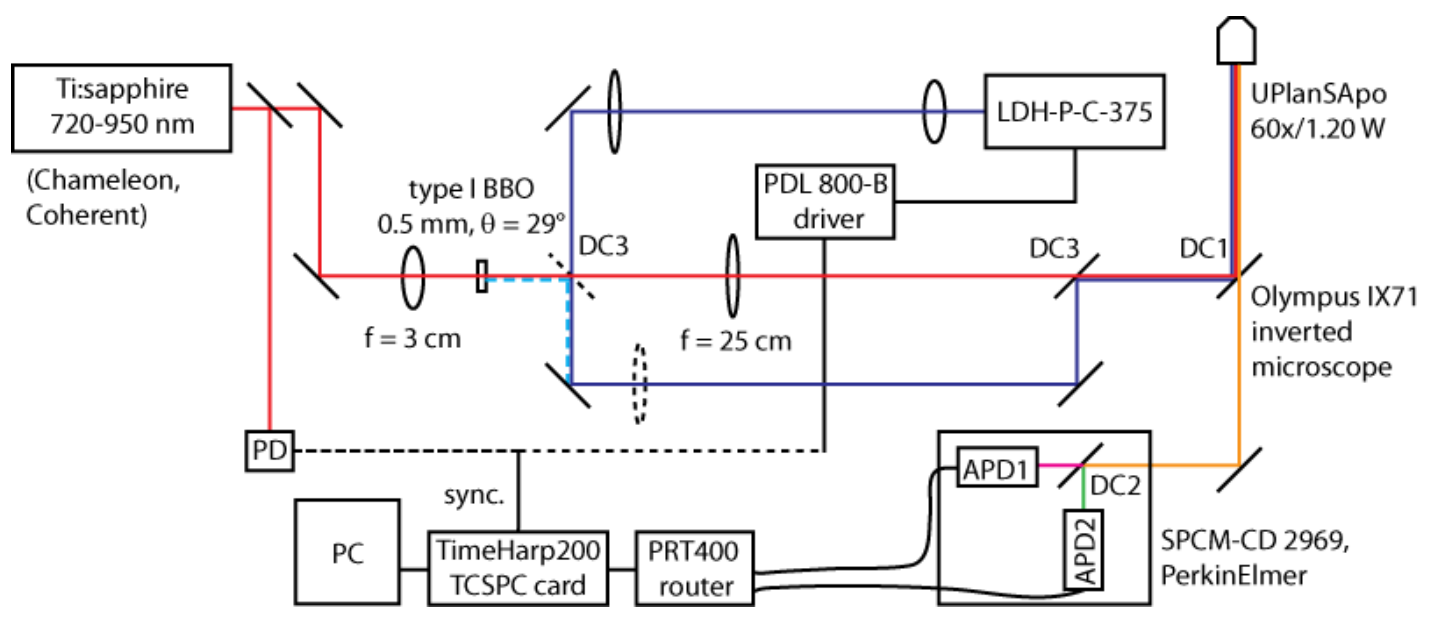

Figure 2.1. Schematic layout of the experimental setup. APD; avalanche photodiode; PD: fast photodiode; DC: dichroic mirror; f: focal length.

A femtosecond Ti:sapphire laser (Chameleon, Coherent) operating at $90 \mathrm{MHz}$ and centered around $800 \mathrm{~nm}$ served as the two-photon excitation source in Chapter 3.1. With photons 
concentrated temporally into pulses 150 fs in width (full-width half-maximum, FWHM) and spatially by the tight-focusing of the objective, simultaneous absorption of two photons may occur if the total energy of the two photons exceeds the energy gap between the $S_{0}$ and $S_{1}$ states. Nevertheless, the initially populated states may be different for two-photon excitation and onephoton excitation at half the wavelength (doubling of photon energy), especially for centrosymmetric molecules. ${ }^{45}$ An advantage of two-photon excitation lies in that the excitation probability away from the focus decreases sharply in both axial and lateral directions, owing to the quadratic dependence on laser intensity.

Whenever two-photon excitation was used, a dichroic mirror 725DCSPXR (AHF) was placed at the position of DC1, in combination with a short pass filter E700SP, to separate excitation (-) and emission (-). The emission was then directed to the left side port of the microscope and split by DC2 (590DCXR, AHF), with longer wavelengths (-) transmitted onto APD1 before passing through a band pass filter D680/30m (Chroma) and shorter wavelengths (-) reflected onto APD2 through the band pass filter D525/20m (Chroma). A variable reflective neutral density filter (not shown) was used to attenuate the excitation power to $25 \mathrm{~mW}$.

To expand the capabilities of the setup, a $0.5 \mathrm{~mm}$ thick type I BBO crystal ( $\beta$-barium borate, $\beta-\mathrm{BaB}_{2} \mathrm{O}_{4}$ ) with $\theta=29^{\circ}$ (Castech) can be flipped into the excitation beam path, at focus, for second-harmonic generation (SHG). The crystal converts a portion of the photons, two-to-one, into photons with doubled energy (half the wavelength). Owing to tunability of the Ti:sapphire laser (720-950 nm), SHG affords one-photon excitation from 360 to $475 \mathrm{~nm}$ (--). The laser fundamental and second harmonics were separated with DC3 (545DCXRU, AHF).

In Chapter 3.2, the measurements were performed primarily with a picosecond (pulse widths $\sim 40$ ps) diode laser (LDH-P-C-375, PicoQuant), which was controlled by the laser driver PDL 800-B (PicoQuant) at a lower repetition frequency of $40 \mathrm{MHz}(-)$ and excitation power of 7.5 $\mu \mathrm{W}$. In this case, as well as when using SHG for excitation, the dichroic mirror 500DCXR (AHF) was inserted as DC1, in combination with a long pass filter AT435lp (Chroma). Signals on APD1 and APD2 were selected by band pass filters D620/20m and D565/20m instead, respectively. All excitation beams were collimated and expanded with a pair of achromatic focusing lenses (focal lengths 3 and $25 \mathrm{~cm}$ ) and set to $10 \mathrm{~mm}$ with an iris diaphragm, in order to overfill the back aperture of the objective $(7.2 \mathrm{~mm})$. The dashed DC3 and 25-cm focusing lens were in place exclusively when SHG was used.

All data were acquired with a PRT 400 router-coupled TCSPC card (TimeHarp200, PicoQuant) and analyzed with the SymPhoTime software (version 5.3, PicoQuant), except for the fitting of FCS curves (elaborated in Section 2.1.2), which was performed with QuickFit $3.0 .^{46}$ 


\subsubsection{Time-correlated single photon counting (TCSPC)}

Most of the results and discussions presented in this work are based on measured timedependent fluorescence intensity decays following the almost instantaneous excitation (with durations of $\sim 150$ fs or $\sim 40 \mathrm{ps}$, depending on the laser used). The decays are proportional to the overall excited-state population, or equivalently the probability of one molecule remaining at the excited state, of either the FRET donor or acceptor (Equations 1.7 or 1.9), and were recorded by time-correlated single photon counting (TCSPC). The principle of TCSPC is classically illustrated in Figure 2.2.

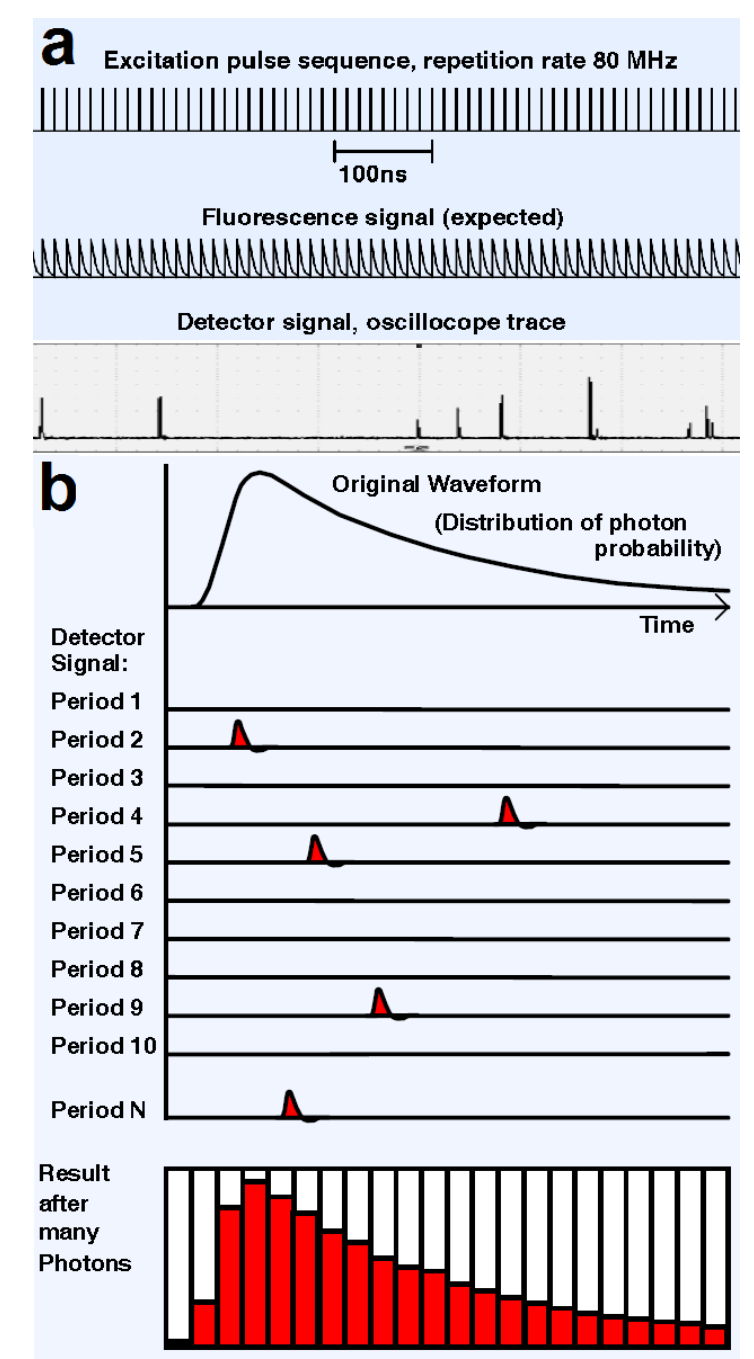

Figure 2.2. Principle of TCSPC. (a) Theoretical effect of the laser pulse trains. (b) Construction of a histogram from single photon events. Adapted from reference 47.

During the time intervals between excitation pulses (11 ns with $90 \mathrm{MHz}$ and 25 ns with 40 MHz repetition frequencies, respectively), the probability of finding a photon decays with time 
in the same way according to Equations 1.7 or 1.9 (Figure 2.2a). In TCSPC, the signal level is generally kept below one-hundredth of the repetition frequency (for example by lowering the laser power or sample concentration) so that no more than one photon is detected per excitation pulse (mostly none). The arrival time of each detected photon with respect to the next incoming laser pulse is registered (reversed mode), and the histogram acquired after the accumulation of many photons reconstructs the probability decay (Figure 2.2b).

The electric output signal from an APD, due to the detection of a photon, starts the charging of a capacitor with a linear voltage ramp (constant charging current), which is stopped by the next synchronization signal from the laser. The stoppage voltage can then be converted to the time difference between the start/stop signals, hence called a time-to-amplitude converter (TAC). With the PRT 400 router, photons arriving at APD1 and APD2 can be counted independently by the TimeHarp200 TCSPC card and binned to time channels 38 ps in width. The synchronization signal when using the Ti:sapphire laser for excitation, either with its fundamental or secondharmonic wavelength, is delivered from a fast photodiode home-built by Prof. Dr. Dirk Schwarzer (Research Group Reaction Dynamics, Max Planck Institute for Biophysical Chemistry), which is illuminated by a split portion of the fundamental (Figure 2.1). As for the diode laser, the electric synchronization output from the laser driver is directly connected to TimeHarp200.

Another important component of a TCSPC module is the constant fraction discriminator (CFD). In contrast to the stable synchronization signal, electric pulses delivered by an APD vary in amplitude (Figure 2.2a, third panel), which will give rise to timing inaccuracies if the pulses are timed with a fixed threshold at the leading edge. A CFD creates a delayed and reverted duplicate of the incoming pulse and adds it to the original one (Figure 2.3). The zero cross point of the sum is independent of incoming pulse amplitudes and used for timing.

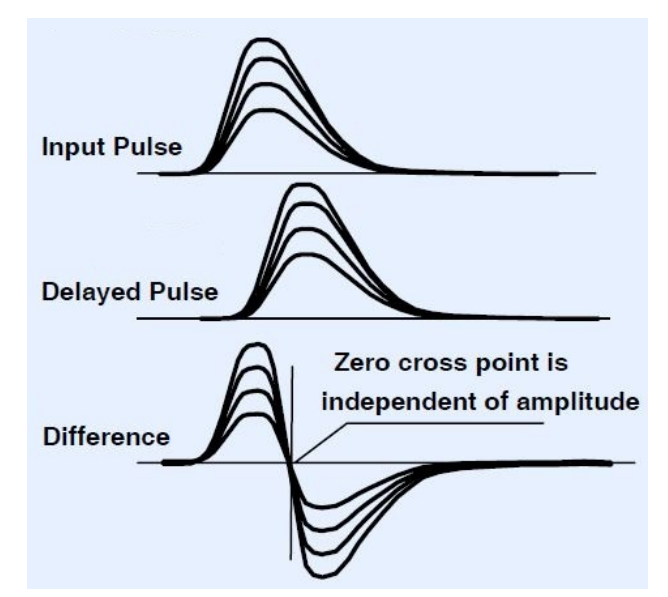

Figure 2.3. The operating principle of CFD. Adapted from reference 47. 
Finally, the reconstructed fluorescence decay curves are fitted with model exponential functions:

$$
F(t)=\sum_{\mathrm{i}} A_{\mathrm{i}} e^{-t / \tau_{\mathrm{i}}}
$$

Time constants $\tau_{\mathrm{i}}$ and amplitudes $A_{\mathrm{i}}$ acquired from the best fits shall then give information on the interactions of a fluorescent molecule with another (e.g., a FRET donor/acceptor pair) or with the surrounding environment (e.g., the solvent).

As exemplified in Figure 2.2b, the observed decay curve begins not abruptly but with a finite slope, which is the outcome of a finite instrument response function (IRF). With either fs Ti:sapphire or ps diode laser as the excitation source, the width of IRF is dominated by the transit time spread (TTS) of the APD detector. The transit time refers to the time between the arrival of a photon and the output electric pulse, which varies with the penetration depth of the photon into the photodiode. The IRF can be imagined as a series of infinitely short pulses generating the same fluorescence decay, the sum of which yields the observed waveform (Figure 2.4). Mathematically, the observed fluorescence intensity at each time $t$ can be expressed as a convolution integral:

$$
F_{o b s}(t)=\int_{0}^{t} F\left(t^{\prime}\right) \operatorname{IRF}\left(t-t^{\prime}\right) d t^{\prime}
$$
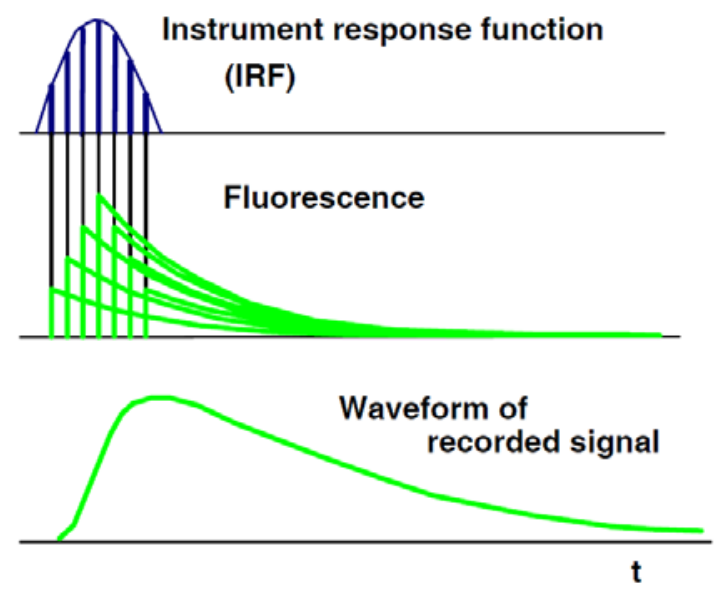

Figure 2.4. Convolution of the fluorescence decay with IRF. Adapted from reference 47.

A proper "synthetic" IRF may be calculated with the SymPhoTime software by taking the derivative of the signal rising edge, given that there is no fast dynamics affecting the fluorescence. For instance, IRF can be acquired first from free Oregon Green 488 dyes in buffer and applied to fit the curves involving more complex dynamics. 


\subsubsection{Fluorescence correlation spectroscopy (FCS)}

In addition to the fluorescence lifetime, recorded photon time traces (e.g., Figure 2.2a, third panel) can also be analyzed with fluorescence correlation spectroscopy (FCS), which provides information on the diffusion behavior and concentration of the fluorescent labels. The autocorrelation function for photons impinging onto either one of the APDs is defined as:

$$
G^{\prime}(\tau)=\frac{1}{T_{\text {total }}} \sum N_{\mathrm{p}}(0) N_{\mathrm{p}}(\tau)
$$

$T_{\text {total }}$ is the total measurement time, and $N_{\mathrm{p}}$ the number of photons. In this context, $\tau$ implies that the whole time trace is shifted by $\tau$ and then compared to itself (with $\tau=0$ ), as depicted in Figure 2.5. With TCSPC, $N_{\mathrm{p}}(0)$ and $N_{\mathrm{p}}(\tau)$ are either 0 or 1 .

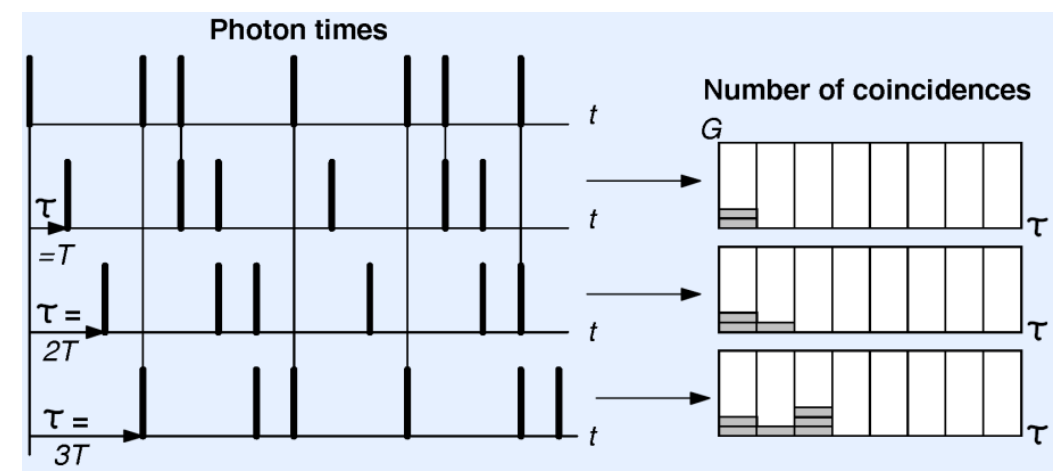

Figure 2.5. Calculation of autocorrelation function $G^{\prime}(\tau)$. $T$ denotes the width of a time channel (38 ps). Adapted from reference 47.

The number of coincidences between the original and shifted time traces are registered at stepwise increased $\tau$ to give the function $G^{\prime}(\tau)$.

A more commonly used expression is the normalized autocorrelation function of fluorescence fluctuations:

$$
G(\tau)=\frac{\langle\delta F(0) \delta F(\tau)\rangle}{\langle F\rangle^{2}}=\frac{G^{\prime}(\tau)}{\langle F\rangle^{2}}-1
$$

with

$$
\delta F=\langle F\rangle-F
$$

The brackets refer to averaging with respect to $T_{\text {total }}$, and thus $<F>$ stands for the average fluorescence intensity.

Fluorescence fluctuations in this study arise from 3D diffusion of fluorescently labeled liposomes in and out of the effective focal volume of: 


$$
V_{\mathrm{eff}, \mathrm{TPE}}=\left(\frac{\pi}{2}\right)^{\frac{3}{2}} r_{0}^{2} z_{0}
$$

using two-photon excitation (TPE). ${ }^{48,49}$ The lateral $\left(r_{0}\right)$ and axial $\left(z_{0}\right)$ dimensions were derived from a 3D Gaussian approximation of the laser intensity profile at position $r$ :

$$
I(r)=I_{0} e^{-2\left(x^{2}+y^{2}\right) / r_{0}^{2}} e^{-2 z^{2} / z_{0}^{2}}
$$

$r_{0}$ and $z_{0}$ specify the distances at which the intensity decreases to $1 / e^{2}$ of its maximum $I_{0}$. With two-photon excitation, the overall detection profile is then given by:

$$
p(r) \equiv I(r)^{2}=I_{0}^{2} e^{-4\left(x^{2}+y^{2}\right) / r_{0}^{2}} e^{-4 z^{2} / z_{0}^{2}}
$$

and accordingly $V_{\text {eff }}$ maps out the boundaries where the detected intensity $p(r)$ decreases to $1 / e^{2}$ from the maximal value $I_{0}^{2}$.

For 3D diffusion, the autocorrelation function $G(\tau)$ can be fitted with the model function:

$$
G(\tau)=\frac{1}{\langle N\rangle} \frac{1}{1+\tau / \tau_{\mathrm{D}}} \frac{1}{\sqrt{1+\left(r_{0} / z_{0}\right)^{2}\left(\tau / \tau_{\mathrm{D}}\right)}}
$$

where $\left\langle N>\right.$ is the average number of diffusing particles in $V_{\text {eff }}$ and $\tau_{\mathrm{D}}$ is the characteristic diffusion time of the particle (e.g., a liposome or a single fluorescent molecule). With twophoton excitation, $\tau_{\mathrm{D}}$ is related to both $r_{0}$ and the size of the particle by:

$$
\begin{gathered}
\tau_{\mathrm{D}}=\frac{r_{0}^{2}}{8 D} \\
D=\frac{k_{\mathrm{B}} T}{6 \pi \eta R}
\end{gathered}
$$

where $T$ is the temperature, $\eta$ the solvent viscosity and $R$ the hydrodynamic radius of the particle, assuming a globular shape. $D$, the diffusion coefficient, is a molecular property and can be utilized to acquire the instrumental parameter $r_{0}$.

When two diffusive species are present, ${ }^{50}$ the model autocorrelation function is given by:

$$
G(\tau)=\frac{N_{1} B_{1}^{2} D_{1}(\tau)+N_{2} B_{2}^{2} D_{2}(\tau)}{\left(N_{1} B_{1}+N_{2} B_{2}\right)^{2}}
$$

where $N_{\mathrm{i}}$ and $B_{\mathrm{i}}$ stand for average particle numbers and the brightness of each species, while $D_{\mathrm{i}}(\tau)$ summarizes the diffusion terms:

$$
D_{i}(\tau)=\frac{1}{1+\tau / \tau_{\mathrm{Di}}} \frac{1}{\sqrt{1+\left(r_{0} / z_{0}\right)^{2}\left(\tau / \tau_{\mathrm{Di}}\right)}}
$$




\subsection{Preparation of sample liposomes}

The standard lipids used in this study were purchased from Avanti Polar Lipids (Figure 2.6), among which DOPC, DOPE, DOPS and DPPTE were synthetic (purity >99\%); PIP2 was extracted from porcine brain, with the structure shown being the predominant species ( $\sim 37 \%)$ in a mixture of different hydrophobic chains, and cholesterol was extracted from wool (purity $>98 \%)$.

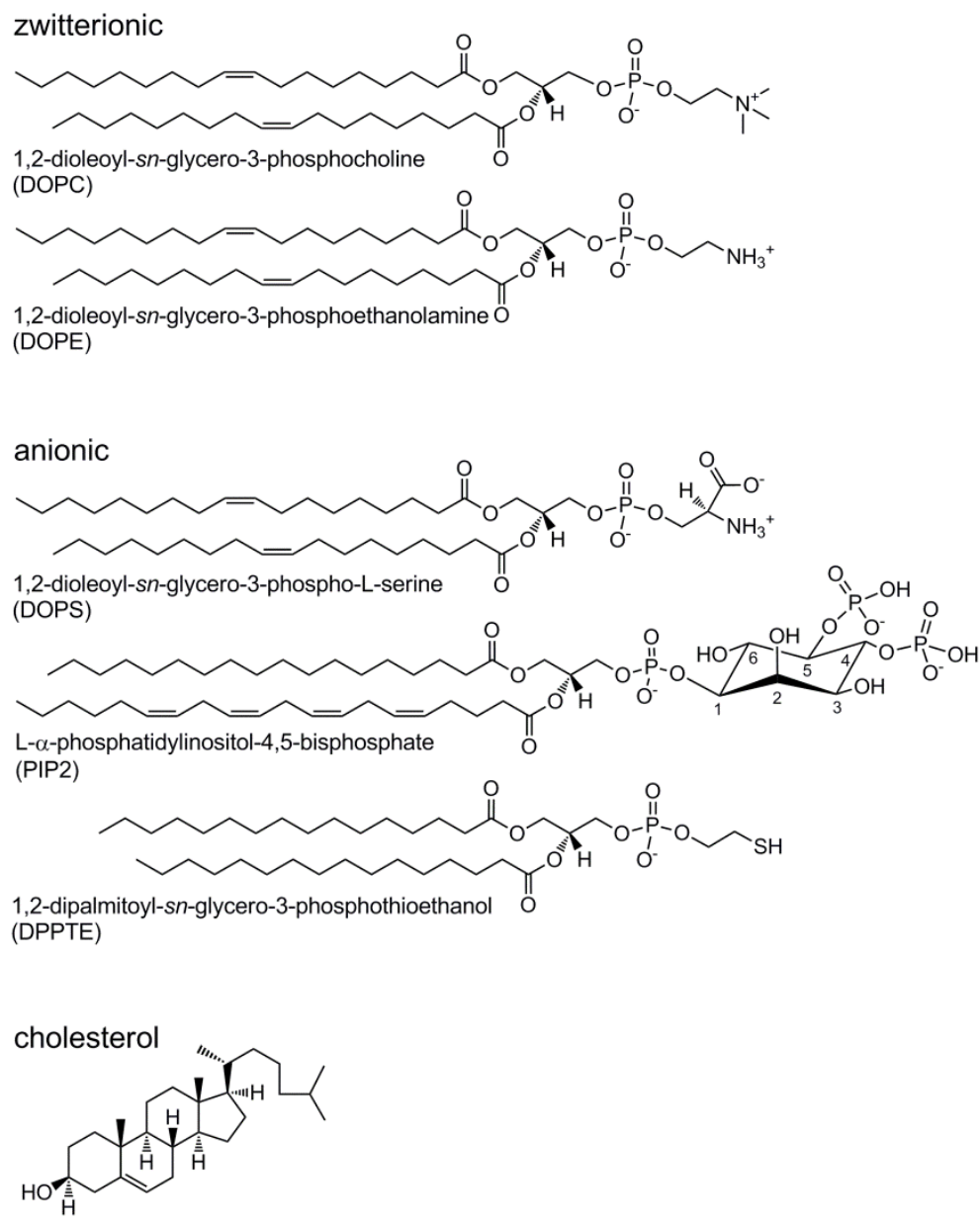

Figure 2.6. Chemical structures of the standard lipids.

The fluorescently labeled lipids were purchased from Molecular Probes (Figure 2.7). Oregon Green and Texas Red are xanthene-based dyes, whereas Marina Blue is a coumarin derivative.

Sample small unilamellar liposomes $(\leq 50 \mathrm{~nm})$ with sizes similar to synaptic vesicles (diameter $\sim 40 \mathrm{~nm}$ ) were prepared as follows: 


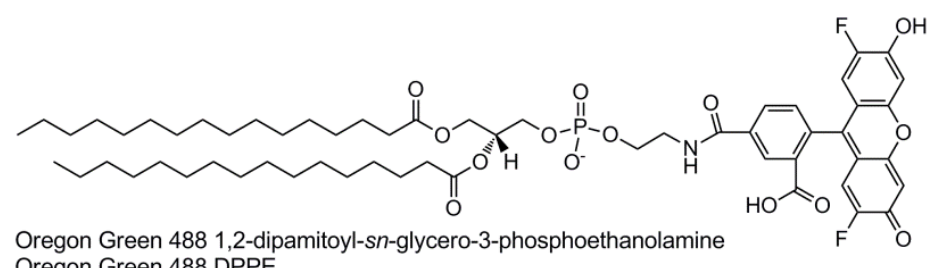
Oregon Green 488 DPPE
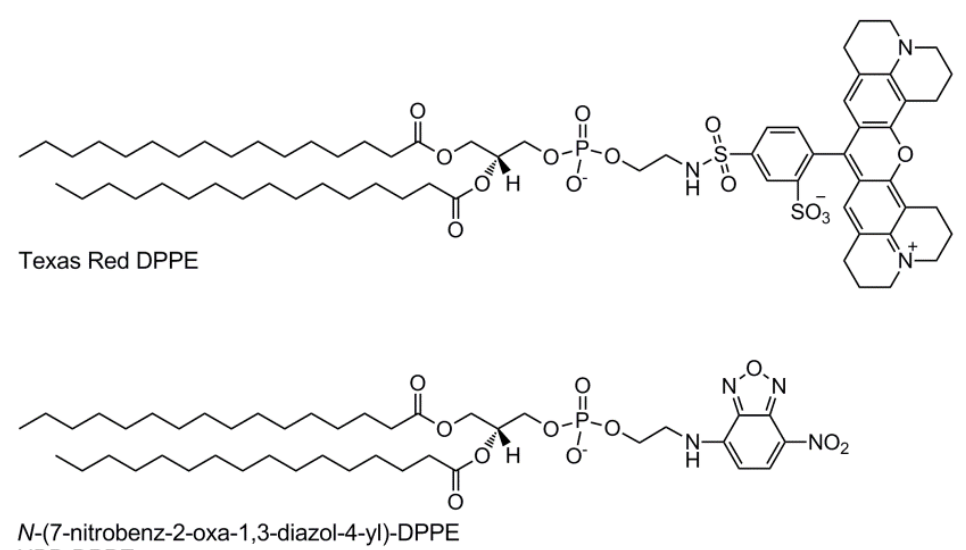

N-(7-nitrobenz-2-oxa-1,3-diazol-4-yl)-DPPE NBD DPPE

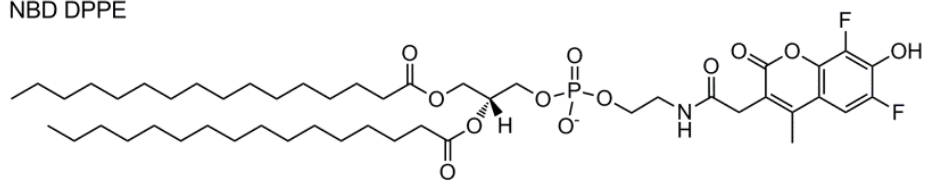

Marina Blue DPPE

Figure 2.7. Chemical structures of fluorescently labeled lipids.

(1) The lipids were dissolved in $\mathrm{CHCl}_{3} / \mathrm{MeOH}$ (volume 2/1) and mixed at the desired compositions. The total number of lipids in a single preparation was $0.55 \mu \mathrm{mol}$.

(2) Purge with a $\mathrm{N}_{2}$ stream to evaporate $\mathrm{CHCl}_{3} / \mathrm{MeOH}$.

(3) Re-dissolve the lipid mixture in $50 \mu \mathrm{L}$ HP150 buffer (KCl 150 mM, HEPES 20 mM, pH 7.4) containing $5 \%$ detergent sodium cholate (by weight).

(4) Add protein or DNA-lipid conjugate solutions to achieve a protein/DNA-to-lipid molar ratio of 1:1000 (Chapter 3.1) or 1:500 (Chapter 3.2).

(5) Load the solution onto a Sephadex G-50 Superfine (Sigma-Aldrich) size-exclusion column and collect the fluorescent fraction of the eluate (tracked with a handheld UV lamp, UVGL25, UVP). Typical concentrations of the eluted liposomes were $\sim 0.4 \mu \mathrm{M}$.

Initially, binary detergent-lipid and ternary detergent-lipid-protein micelles (monolayered) coexisted in equilibrium with detergent monomers. While moving down the column, the detergent monomers trailed behind, and the detergents in mixed micelles were gradually depleted to maintain the equilibrium. Subsequently, micelles began to merge, in order to decrease the high curvature unfavorable to the longer-tailed lipids. Finally a phase transition was made to form bilayered liposomes (Figure 2.8). 


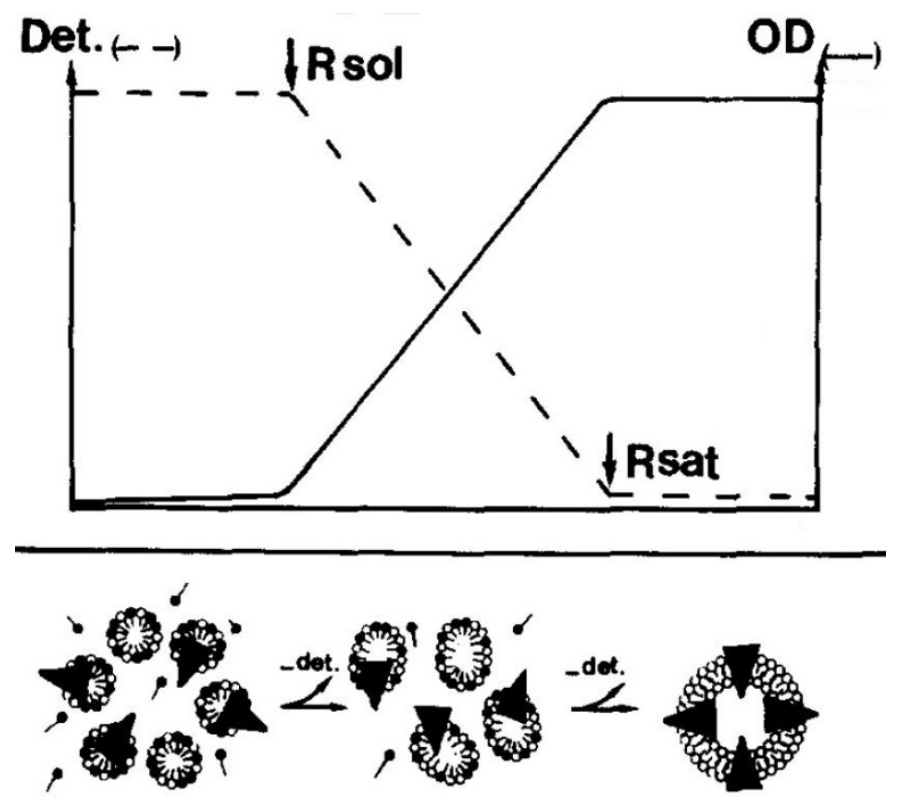

Figure 2.8. Mechanism of liposome formation using size-exclusion chromatography and cholate as the detergent. $(\bullet-)$ Detergents, $\left(\mathrm{O}^{-}\right)$lipids and $(\boldsymbol{\nabla})$ proteins. OD (optical density) increases with the concentration of liposomes. Rsat denotes the saturating detergent-to-lipid ratio in liposomes, above which mixed micelles and liposomes begin to coexist; whereas Rsol is the ratio when lipids become completely solubilized into mixed micelles. Adapted from reference 51, copyright (1995), with permission from Elsevier.

The HP150 buffer was used throughout Chapter 3.2. As for Chapter 3.1, 1 mM EGTA was included to buffer $\mathrm{Ca}^{2+}$ (HP150-EGTA). For $\mathrm{Ca}^{2+}$-activation of syt-1, syt-1 proteoliposomes were first incubated with target liposomes in HP150-EGTA for 30 min. Afterwards, $150 \mu \mathrm{L}$ HP150-EGTA with $1.467 \mathrm{mM} \mathrm{CaCl}_{2}$ was added to the mixture and the final volume was adjusted to $200 \mu \mathrm{L}$ with extra HP150-EGTA. The total $\mathrm{Ca}^{2+}$ concentration was then $1.1 \mathrm{mM}$, with $100 \mu \mathrm{M}$ being free (checked with Fluo-5N, ${ }^{52}$ Molecular Probes). Another $30 \mathrm{~min}$ of incubation allowed for the $\mathrm{Ca}^{2+}$-binding effects to reach equilibrium.

\subsubsection{Protein constructs}

Full-length syt-1 (amino acids 1-421) wild-type and mutants were purified by Dr. Angel Pérez-Lara, and the SNARE proteins by Ursel Reis (Department of Neurobiology, Max Planck Institute for Biophysical Chemistry). Three previously characterized syt-1 $\mathrm{Ca}^{2+}$-binding mutants were used: $a * B$ (D178A, D230A and D232A), Ab* (D309A, D363A and D365A) and a*b* (D178A, D230A, D232A, D309A, D363A and D365A), in which $\mathrm{Ca}^{2+}$ binding to C2A, C2B or to both C2 domains was disrupted (Figure 1.6) by turning negatively charged aspartate into neutral alanines. ${ }^{37}$ 
In Chapter 3.2, full-length synaptobrevin-2 (1-116, Figure 1.5a) was reconstituted into one type of liposomes. The other type of liposomes included the $\Delta \mathrm{N}$ acceptor complex, which consisted of a truncated syntaxin-1A (183-288), a SNAP-25A (1-206, C84S, C85S, C90S and C92S) with all cysteines mutated to serines, and a soluble C-terminal portion of the synaptobrevin-2 SNARE motif $\left(\mathrm{syb}_{49-96}\right)$. The $\mathrm{syb}_{49-96}$ peptide was first introduced in reference 42, which greatly accelerated the slow reconstituted fusion (compared to that in vivo) by preventing the formation of an inhibitory syntaxin-1A/SNAP-25A 2:1 complex. It was subsequently displaced by full-length synaptobrevin-2 during full-zippering. Yet this displacement step may still be rate-limiting in the observed fusion reaction.

\subsubsection{Synthesis and characterization of lipid-anchored DNA oligonucleotides}

The six lipid-anchored DNA oligonucleotides (Table 2.1) used in Chapter 3.1 were designed and synthesized by Prof. Dr. Claudia Höbartner and Jan Seikowski (Research Group Nucleic Acid Chemistry, Max Planck Institute for Biophysical Chemistry).

Table 2.1. Sequences and MS analysis of the titled oligonucleotides.

\begin{tabular}{|c|c|c|c|}
\hline & $\begin{array}{l}\text { 5'-Sequence-3' } \\
\text { X=1,2-O-dioctadecyl-(rac)-glycerol }\end{array}$ & $\begin{array}{l}\text { Mol. Wt. } \\
\text { calculated } \\
\text { [g/mol] }\end{array}$ & $\begin{array}{l}\text { Mol. Wt. } \\
\text { found } \\
{[\mathrm{g} / \mathrm{mol}]}\end{array}$ \\
\hline 10a & XGACCTCGCAG & 3671.8 & 3671.5 \\
\hline $10 \mathrm{~b}$ & XCTGCGAGGTC & 3702.8 & 3703.3 \\
\hline 15a & XGACCTCGCATCGTGT & 5202.9 & 5203.3 \\
\hline 15b & XATACGATGCGAGGTC & 5275.8 & 5276.2 \\
\hline $25 a$ & XTCGACACGGAAATGTTGAATACTAC $^{53}$ & 8333.0 & 8333.4 \\
\hline $25 b$ & XGTAGTATTCAACATTTCCGTGTCGA & 8306.0 & 8306.8 \\
\hline
\end{tabular}

The oligonucleotides were synthesized under standard solid-phase DNA synthesis conditions, ${ }^{54}$ except for using 1,2-O-dioctadecyl-(rac)-glycerol 3-(2-cyanoethyl) $N, N$ diisopropylphosphoramidite as the last base. The lipid-phosphoramidite was prepared using 1,2$O$-dioctadecyl-(rac)-glycerol (Chem-Impex) and 2-cyanoethyl $N, N$-diisopropylchlorophosphoramidite in the presence of $\mathrm{N}$, $\mathrm{N}$-diisopropylethylamine (Hünig's base) in $\mathrm{CH}_{2} \mathrm{Cl}_{2}$ (structures shown in Figure 2.9). ${ }^{55}$ All phosphoramidites were dissolved in anhydrous $\mathrm{CH}_{2} \mathrm{Cl}_{2}$ 
(100 $\mathrm{mM}$ ) and the coupling time was $2 \mathrm{~min}$, except for the lipid-phosphoramidite (12 min). The final DNA oligonucleotides were deprotected and cleaved from the solid support with $\mathrm{NH}_{4} \mathrm{OH} / \mathrm{EtOH}\left(3: 1,55^{\circ} \mathrm{C}, 20 \mathrm{~h}\right)$, and purified by RP-HPLC on a Resource 15RPC column (1 $\mathrm{mL})$, using a linear gradient of $\mathrm{CH}_{3} \mathrm{CN}$ in triethyl ammonium acetate buffer $(0-100 \%$ in 20 column volumes). Purity and identity of the products were confirmed by HPLC and ESI-MS (Table 2.1).

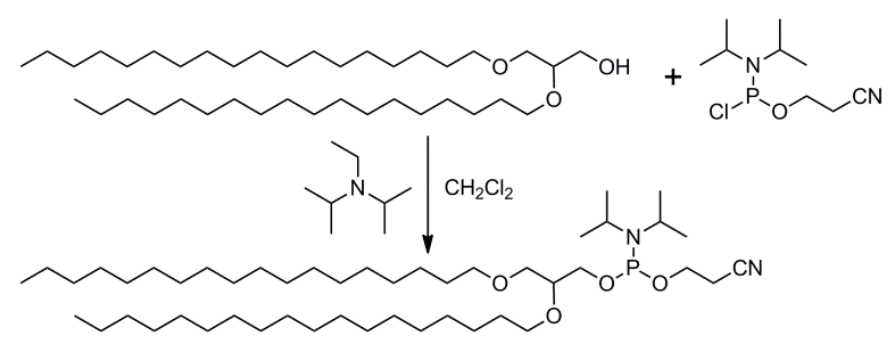

Figure 2.9. Synthesis of the lipid-phosphoramidite.

Hybridization of the DNA-liposomes was achieved by incubation for 5 min at $35{ }^{\circ} \mathrm{C}$ for 10 bp and $55{ }^{\circ} \mathrm{C}$ for 15 and 25 bp, followed by slow cooling back to room temperature. The procedure was analogous to typical annealing protocols for oligonucleotides. Namely, the selected temperatures were close to the melting temperatures $\left(T_{\mathrm{m}}\right)$ of the strands (calculated with OligoAnalyzer 3.1, http://www.idtdna.com/calc/analyzer, Integrated DNA Technologies), which are 34.8, 52.9 and $59.6{ }^{\circ} \mathrm{C}$ for 10,15 and $25 \mathrm{bp}$, respectively.

\subsubsection{Asymmetrically labeled liposomes}

As mentioned briefly in Section 1.2.1, the "dequenching" assay, in which the FRET donor and acceptor labels are first included in the same type of liposomes, are frequently used to monitor lipid mixing resulting from membrane fusion. A common combination of dyes is with NBD being the donor and rhodamine B as the acceptor (e.g., in references 15 and 17). Remarkably, NBD on the outer leaflet can be reacted with dithionite ions $\left(\mathrm{S}_{2} \mathrm{O}_{4}{ }^{2-}\right)$ to become non-fluorescent, so the increase of NBD fluorescence corresponds to the extent of fusion of the inner leaflet alone. Since membrane fusion starts with the outer leaflet (Figure 1.4), the kinetics of inner leaflet fusion is considered to be slower than, if not the same as, the overall fusion rate when both leaflets are labeled. Such a comparison must be drawn with two separate experiments (with and without $\mathrm{S}_{2} \mathrm{O}_{4}{ }^{2-}$ treatment) and proper normalization. ${ }^{56}$ If, however, the two leaflets are labeled differently and distinguishably, fusion of the outer and inner leaflets can be recorded and compared simultaneously. 
The asymmetrically labeled liposomes in Chapter 3.2 were prepared with the following steps, starting with liposomes containing 2\% NBD DPPE and 0.5\% thiol-functionalized DPPTE:

(1) Add the eluted liposomes to an excess of Oregon Green 488 maleimide in powder form (54 nmol, Molecular Probes), and react for 2 hours at room temperature.

(2) Mix with an equal volume of freshly prepared $\mathrm{Na}_{2} \mathrm{~S}_{2} \mathrm{O}_{4} 200 \mathrm{mM}$ (reference 57) in HP150 buffer. React for 5 minutes at room temperature.

(3) Load the solution onto Sephadex G-50 Superfine columns (with no more than $200 \mu \mathrm{L}$ per column) and collect the first fluorescent fraction (the second slowly traveling band would be the excess dyes). This step shall remove both the excess dyes and $\mathrm{Na}_{2} \mathrm{~S}_{2} \mathrm{O}_{4}$.

The first chemical reaction (Figure 2.10a) is essentially the same as labeling cysteine residues of proteins, ${ }^{58}$ while the second reaction reduces the nitro-substituent of NBD to an amine (Figure 2.10b). Since the lowest-lying transition (centering around $460 \mathrm{~nm}$ ) of the NBD molecule involves charge transfer from the amine lone pair electrons (non-bonding orbital) to the nitrogroup $\pi^{*}$-antibonding orbital, reduction of the nitro-group eliminates this transition and the green fluorescence disappears. ${ }^{59}$ The order of reactions cannot be reversed, as excess $\mathrm{Na}_{2} \mathrm{~S}_{2} \mathrm{O}_{4}$ would compete with DPPTE to reduce the maleimide $\mathrm{C}=\mathrm{C}$ bond.$^{60}$ For fusion experiments, fulllength synaptobrevin-2 was reconstituted into the precursor NBD/DPPTE liposomes. Synaptobrevin-2 bears merely one cysteine (C102) buried in the transmembrane domain, which should not interfere with the reactions.

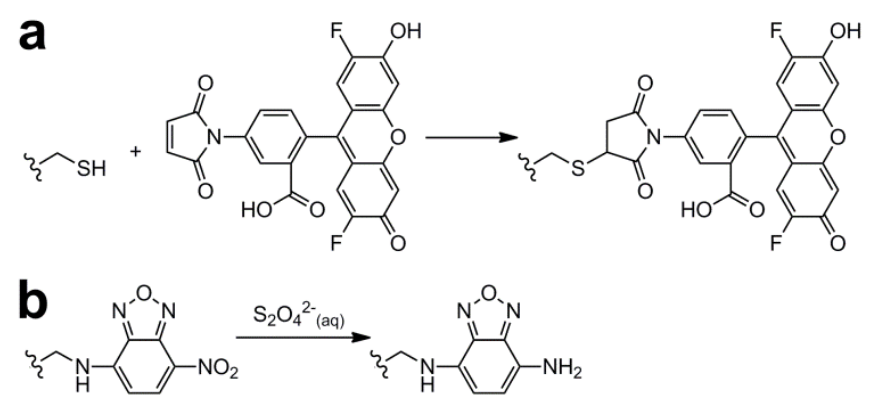

Figure 2.10. The one-pot, two-step reaction to produce asymmetrically labeled liposomes.

The two labels, NBD and Oregon Green 488, were differentiated by their different fluorescence lifetimes (Section 3.2.1). 


\subsection{Fabrication of microfluidic channels}

The microfluidic channels (Figure 2.11) used in Chapter 3.2 were designed and fabricated by Hsin-Fang Hsu (Laboratory for Fluid Dynamics, Pattern Formation and Biocomplexity, Max Planck Institute for Dynamics and Self-Organization). The channel was composed of a glass coverslip (24 × $60 \mathrm{~mm}, 0.13-0.16 \mathrm{~mm}$ thickness, Menzel-Gläser) and a polydimethylsiloxane block (PDMS, Sylgard 184, Dow Corning). The indented channel structure was produced with a reusable silicon wafer template (Si-Mat) bearing the complementary pattern on top, which was made with standard soft lithography ${ }^{61}$ using the SU-8 3025 photoresist (MicroChem). The pattern was drawn with the LibreCAD software (http://librecad.org) and printed to a photoemulsion/film photomask (resolution 50,800 dpi, Selba S.A.). Before assembling the PDMS block and the glass coverslip, they were both treated with air plasma for 30 seconds (plasma cleaner PDC-002, Harrick Plasma), which rendered the surfaces hydrophilic. To prevent adhesion of liposomes onto channel surfaces, the channels were stored for at least two days before use for them to become hydrophobic. For each liposome preparation, a new channel was fabricated.

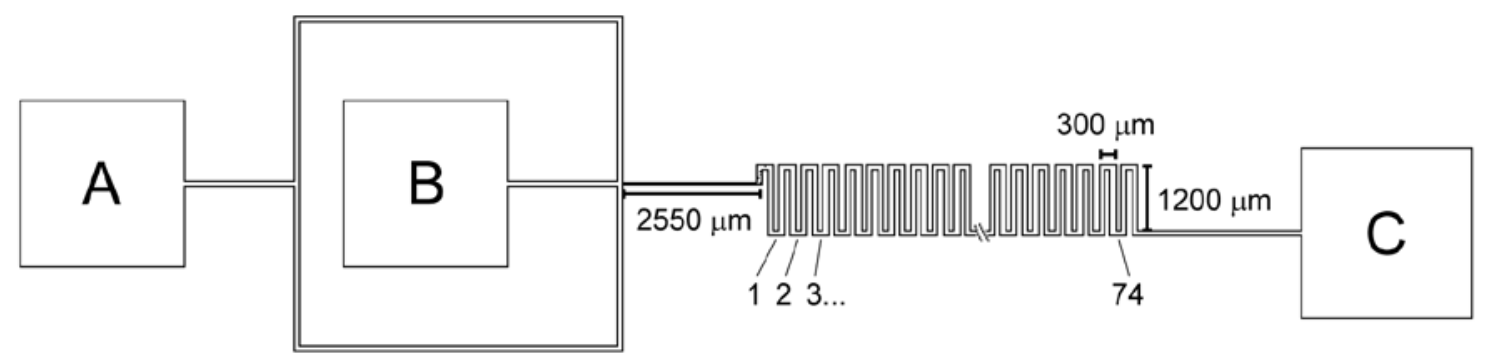

Figure 2.11. The design of the microfluidic channel. The middle segments were truncated $(\backslash \backslash$ ) for better visualization of the fine structures. A and B are the inlets connected to syringes, whereas $\mathrm{C}$ is connected to waste. Courtesy of Hsin-Fang Hsu.

The channel was on average $95 \mu \mathrm{m}$ in height $(90-100 \mu \mathrm{m})$ and $100 \mu \mathrm{m}$ in width, except for the $2550 \mu \mathrm{m}$ long mixing segment, which was narrowed to $50 \mu \mathrm{m}$. Pillars $16 \mu \mathrm{m}$ in diameter were randomly placed within the mixing segment to induce turbulences that facilitated faster mixing. There were a total of $74300 \mu \mathrm{m}$ long horizontal segments in the lower part, marked with numbers which could be read under the microscope (not shown). An F-View II CCD (charge-coupled device) camera mounted on top of the binocular port of the microscope, along with the analySIS getIT software (Olympus), was used to place the laser focus at the center of these segments or their upper counterparts. Blue donor (Marina Blue) and green acceptor 
(Oregon Green 488 and NBD) liposomes were loaded separately into Hamilton gastight glass syringes (500 $\mu \mathrm{L}$, inner diameter $3.26 \mathrm{~mm}$ ) and injected into the channel through inlets A and $\mathrm{B}$, respectively. The flow was controlled by a two-rack syringe pump (PHD 2000, Harvard Apparatus) and set to $20 \mu \mathrm{L} / \mathrm{hr}$. Accordingly, the time of travel between two adjacent numbered segments was 5.4 seconds, the same as the time required to pass through the narrowed neck. In other words, the samples in the succeeding downstream segment are mixed (reacted) for 5.4 more seconds. In the ensuing Results and Discussion, the lower horizontal segment 1 is defined as time zero (0 s) when the donor and acceptor liposomes become thoroughly mixed. 


\section{RESULTS AND DisCUSSION}

\subsection{Control of membrane gaps by synaptotagmin-Ca ${ }^{2+}$ measured with a novel membrane distance ruler}

This part of work (except for Section 3.1.4) has been published in Nat. Commun. 2014, 5, 5859 (doi: 10.1038/ncomms6859), ${ }^{62}$ under a Creative Commons Attribution 4.0 International License (CC BY). Herein, modifications were made to the texts and figure captions.

The aim was to verify whether synaptotagmin-1 (syt-1) reduced the distance between two membranes upon $\mathrm{Ca}^{2+}$-activation as proposed in reference 43 (Section 1.2.2), and to do so by measuring the membrane distances accurately at a nm scale. The membrane distances were probed with multiple inter-membrane FRET interactions between small unilammelar liposomes labeled with $0.5 \%$ Oregon Green 488 DPPE or $1 \%$ Texas Red DPPE (molar ratios) as the donor or acceptor liposomes, respectively. The fluorescence lifetime of the donor dye Oregon Green was chosen as a robust indicator of FRET (Equation 1.7), as it is insensitive to variations in the concentration of liposomes within the focal volume of $\sim 1 \mathrm{fL}$ (Equation 2.5) or to variations in alignment conditions of the experimental setup.

In contrast to FRET-based distance measurements within or between proteins, ${ }^{63,64}$ distances between two membranes cannot be easily extracted. In proteins, single donor and acceptor dyes are attached to defined sites and a single donor-acceptor pair is distant from the other fluorophores. In these cases, the average distance between the pair can be easily derived, providing that the Förster distance $R_{0}$ for the selected donor and acceptor is known. On liposomes, contrarily, the fluorophores are distributed across the surfaces of both the inner and outer leaflets. Hence, in a single tethered cluster, each donor molecule may transfer the excitation energy to multiple acceptors, and the probability of each donor undergoing FRET is different and depends on its position relative to the acceptor liposomes, which in turn leads to 
different lifetimes (Figure 3.1a). Therefore, it is crucial to measure first a calibration curve using tethered liposomes with predefined spacings so that the donor fluorescence lifetimes can be correlated properly with membrane distances between donor and acceptor liposomes.

\subsubsection{The membrane distance ruler}

The calibration of such a membrane distance ruler was done via using membrane-anchored double-stranded DNA (dsDNA) of various well-defined lengths as discrete spacers (Figure 3.1). ${ }^{53}$ In general, dsDNA shorter than its persistence length of $50 \mathrm{~nm}$, or 150 base pairs (bp), is known to have high bending rigidity. ${ }^{65}$ Herein three different lengths well below the persistence length were synthesized (Section 2.2.2 and Table 2.1), $3.3 \mathrm{~nm}$ (10 bp), $5.0 \mathrm{~nm}$ (15 bp) and 8.3 $\mathrm{nm}$ (25 bp). The complementary strands were then individually reconstituted into donor and acceptor liposomes, respectively, with a 1:1000 DNA-to-lipid molar ratio. 10\% anionic lipids DOPS were included in both types of DNA liposomes, with remaining lipids adjusted with DOPC to yield $100 \%$. The net negative charges on the liposome surfaces and the negatively charged DNA backbones helped to stabilize the DNA in a perpendicular orientation, which was already observed when there were no repulsive charges on the membranes. ${ }^{66}$

a

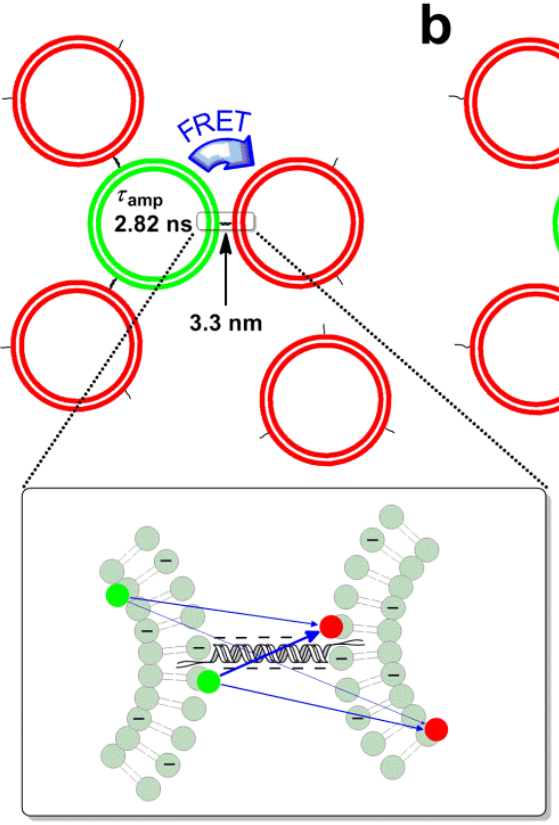

C

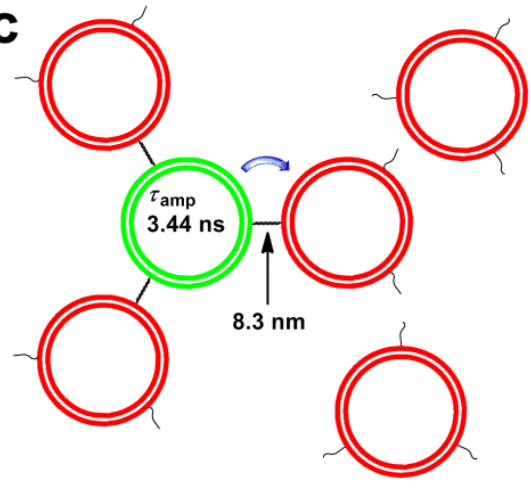

d 10a: XGACCTCGCAG

15a: XGACCTCGCATCGTGT

15b : CTGGAGCGTAGCATAX

25a: XTCGACACGGAAATGTTGAATACTAC

25b : AGCTGTGCCTTTACAACTTATGATGX

Figure 3.1. FRET from green donor liposomes to the surrounding red acceptor liposomes. The upper panels (a-c) show the experimental design of the membrane distance ruler for small unilamellar liposomes, with the spacings specified. FRET decreases as the distance increases, as reflected in the longer amplitude-weighted lifetime $\left(\tau_{\mathrm{amp}}\right)$. In $(\mathbf{d})$ the DNA sequences are listed again in a complementary manner. 
Figure 3.1 also illustrates that, in the experimental design, an excess of acceptor liposomes was used to drive the equilibrium so that each donor liposome is surrounded by an equal number of acceptor liposomes, in order to eliminate the contribution of differences in the degree of tethering. ${ }^{67}$ Otherwise, an observed reduction in the donor lifetime might be caused by an increased number of bound acceptor liposomes, instead of a decrease in distance. To determine the required ratio, the acceptor liposome concentration was increased gradually while the donor liposomes were kept at one liposome in the focal volume $(\sim 1.5 \mathrm{nM})$. The donor fluorescence decay curves converged after the ratio exceeded 1:10 (Figure 3.2). As a result, a ratio of 20 acceptor liposomes to 1 donor liposome was selected for ensuing analysis and discussions.
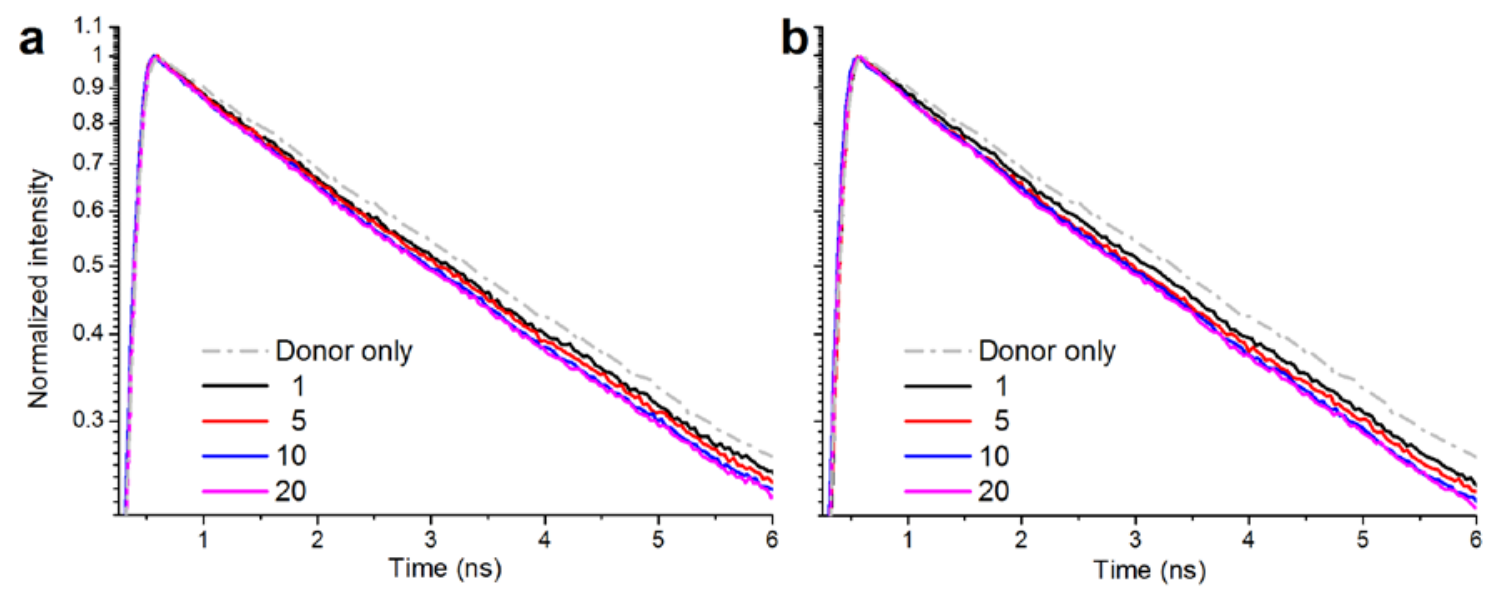

Figure 3.2. Convergence of the fluorescence decay curves at higher excess ratios.

Shown for (a) Syt-1 samples in 1 mM EGTA and (b) in $100 \mu \mathrm{M} \mathrm{Ca}^{2+}$. The numbers indicate the ratio of acceptor liposomes relative to the donor liposomes.

The fluorescence decay curves of the three DNA-tethered liposome mixtures were measured with TCSPC (Section 2.1.1) and the results are summarized in Figure 3.3a. The emission wavelength was chosen at the blue edge of donor fluorescence spectrum (525 $\pm 10 \mathrm{~nm})$ to (i) minimize the crosstalk between donor and acceptor channels (Figure 3.4) and (ii) to selectively amplify the contributions of donor molecules facing the acceptor liposomes (elaborated in Section 3.1.4). The three curves were clearly distinguishable and decayed faster than the control sample without DNA. They were fitted with two exponential components:

$$
F(t)=A_{1} e^{-t / \tau_{1}}+A_{2} e^{-t / \tau_{2}}
$$

and the amplitude-weighted lifetime ( $\left.\tau_{\text {amp }}\right)$, was then calculated:

$$
\tau_{\mathrm{amp}}=\frac{A_{1}}{A_{1}+A_{2}} \tau_{1}+\frac{A_{2}}{A_{1}+A_{2}} \tau_{2}
$$



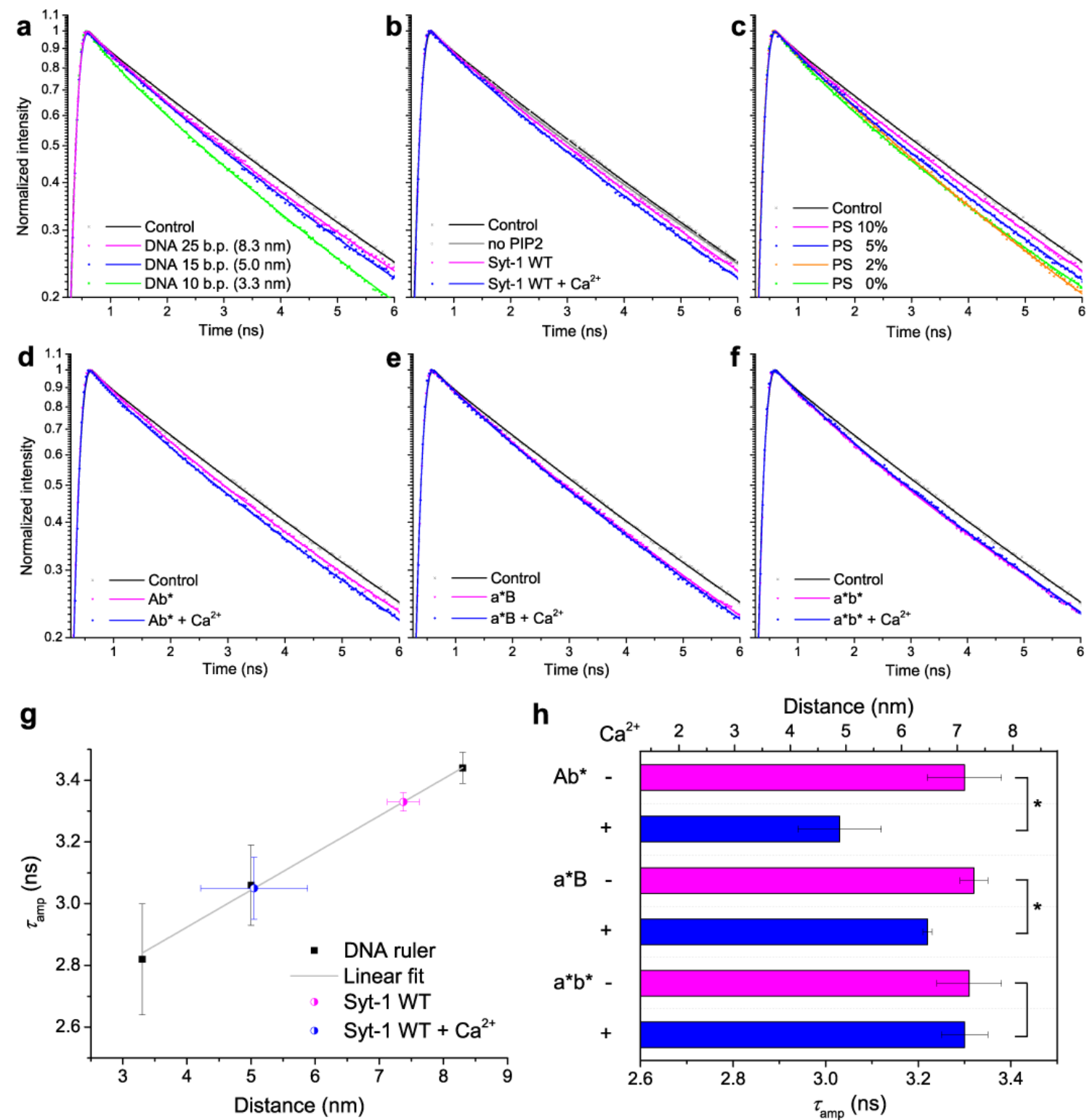

Figure 3.3. The measured fluorescence decay curves and the membrane distance ruler for converting fitted $\tau_{\text {amp }}$ into closest distances between small unilamellar liposomes. Decay curves for (a) DNA-tethered liposomes with specified number of base pairs and (b) syt-1 wild-type (WT) reconstituted into donor liposomes in the absence and presence of $100 \mu \mathrm{M} \mathrm{Ca}^{2+}$. No PIP2 indicates the sample in which $2 \%$ PIP2 was substituted with 7\% PS. (c) Measurements without $\mathrm{Ca}^{2+}$ for syt-1 WT reconstituted into donor liposomes with various molar ratios of PS. (d-f) Decay curves for $A b^{*}, a^{*} B$ and $a^{*} b^{*}$ mutants. Control stands for the experiments in which there was no protein or DNA on either type of liposomes. The symbols represent experimental data, whereas the lines are reconvolution fits using two exponentials. (g) The plot of $\tau_{\text {amp }}$ versus distance. A linear regression line can be constructed with the DNA-tethered liposomes and applied to determine the distance between syt-1 tethered liposomes. (h) $\tau_{\mathrm{amp}}$ and the calculated 
distance information for the mutants. The error bars represent s.d. values obtained from three independent repeats. Asterisks $\left(^{*}\right)$ designate when the $\tau_{\text {amp }}$ values are statistically different $(\mathrm{P}<0.05$, two-tailed unpaired t-test).

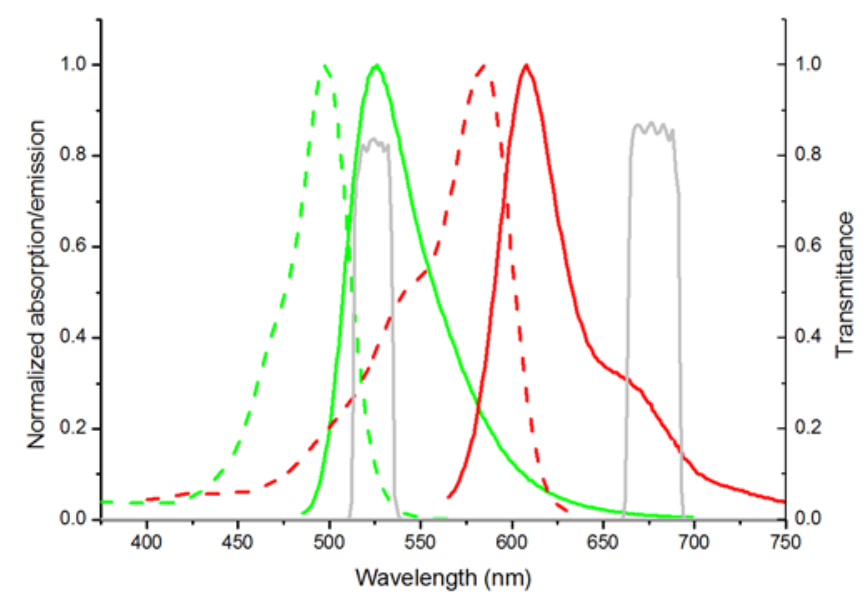

Figure 3.4. Spectra of the fluorophores and band pass filters. Spectra of the FRET donor, Oregon Green 488, are shown in green and those of the acceptor, Texas Red, in red. The dashed lines are the absorption and solid lines the fluorescence emission. The gray solid lines indicate the transmittance of band pass filters D525/20m and D680/30m.

By measuring three independent replicates, $\tau_{\mathrm{amp}}$ for liposomes with membrane distances of 8.3, 5.0 and $3.3 \mathrm{~nm}$ was determined to be $3.44 \pm 0.05$, $3.06 \pm 0.13$ and $2.82 \pm 0.18 \mathrm{~ns}$, respectively (average \pm s.d.). A plot of $\tau_{\text {amp }}$ versus the membrane distance could be very well fitted by a linear approximation (Figure 3.3g), which later served as the calibration curve for membrane distance determination. As control, hybridization of complementary DNA strands on the liposomes was inhibited by adding a free single strand (without the lipid anchor, Figure 3.5). Under these conditions, the lifetime was restored to the control level observed with a sample containing donor and excess acceptor liposomes, but neither with DNA.

An advantage of the calibration procedure is that any nonspecific effects, such as nonspecific liposome clustering, are intrinsically corrected for. To quantify the extent of such effects, a sample containing only pure donor liposomes was compared with the donor-acceptor control (Figure 3.5). This comparison provides evidence that nonspecific effects contributed merely to a minor extent to the overall fluorescence decay. 


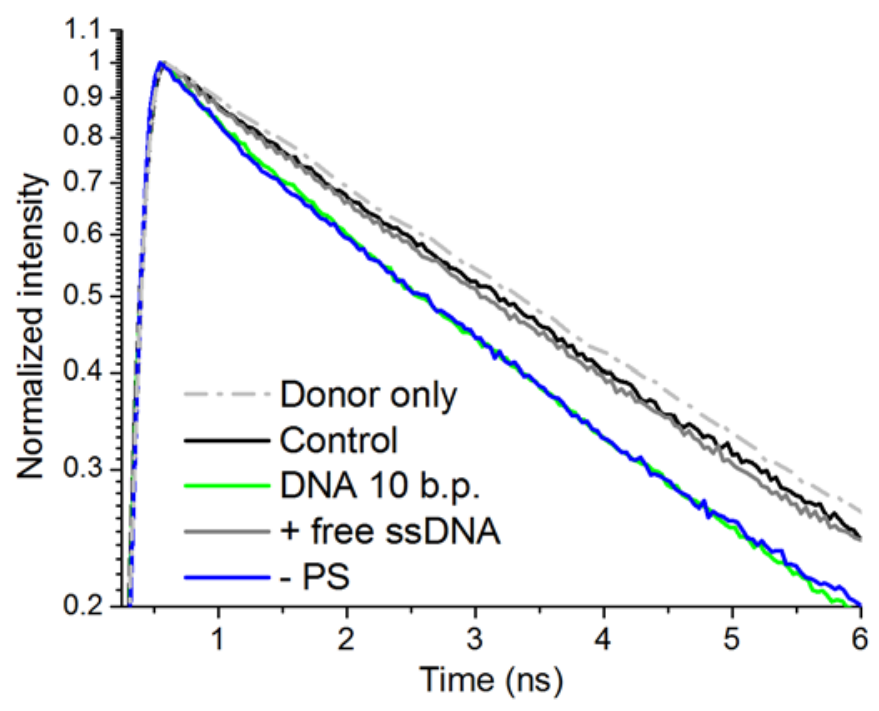

Figure 3.5. Inhibition of DNA-liposomes hybridization via the addition of a free single strand. Shown for the 10 b.p. samples. The additional blue curve represents the sample in which the negative charges on both types of liposomes were omitted and the gray dashed dotted curve was measured with pure donor liposomes.

\subsubsection{Synaptotagmin-1 controls the gap between two membranes}

Next, full-length syt-1 was reconstituted with a 1:1000 protein-to-lipid molar ratio into donor liposomes containing 10\% DOPS (20\% DOPE, 10\% cholesterol and adjusted with DOPC to $100 \%$ ), and the fluorescence decay was measured when they were bound to acceptor liposomes bearing 15\% DOPS plus 2\% PIP2 (also with 20\% DOPE, 10\% cholesterol and DOPC). In the absence of $\mathrm{Ca}^{2+}$ (1 mM EGTA), $\tau_{\text {amp }}$ was close to that of the $8.3 \mathrm{~nm}$ DNA-tethered liposomes (3.33 $\pm 0.03 \mathrm{~ns}$ ) and the membrane distance was estimated to be $7.4 \mathrm{~nm}$ (Figure 3.3b,g). To verify that the decrease in fluorescence lifetime compared with the control sample was caused by the specific interaction of the poly-lysine patch (KKKK) with PIP2, the poly-anionic PIP2 (2\%) in the acceptor liposomes was replaced by increasing the concentration of mono-anionic PS by 7 to $22 \%$ PS, which maintained the net negative charge of the acceptor liposomes. ${ }^{68}$ Without PIP2, the fluorescence lifetime returned to the control level, showing that tethering to the syt-1 proteoliposomes was abolished. The incubation was repeated using PIP2-containing liposomes in the absence of $\mathrm{Ca}^{2+}$ (1mM EGTA) to allow for the KKKK-PIP2 binding and appropriate $\mathrm{Ca}^{2+}$ buffer was added afterwards to achieve a final free $\mathrm{Ca}^{2+}$ concentration of 100 $\mu \mathrm{M}$ (Section 2.2). The resulting fluorescence decay was faster and corresponded to that measured with liposomes tethered by the DNA-ruler at a distance of $5.0 \mathrm{~nm}\left(\tau_{\mathrm{amp}}=3.05 \pm 0.10\right)$. It is concluded accordingly that the gap between syt-1 proteoliposomes and the surrounding acceptor liposomes decreases to $\sim 5 \mathrm{~nm}$ after the addition of $\mathrm{Ca}^{2+}$. 
The distance of $7.4 \mathrm{~nm}$ in the absence of $\mathrm{Ca}^{2+}$ is relatively short, suggesting that the linker between the transmembrane domain and the C2A domain is not completely stretched (Figure 1.6). Intriguingly, the linker contains a membrane-adjacent stretch of positive charges, which may interact with the more C-terminally localized stretch of negative charges ${ }^{69}$ and/or with anionic lipids in the resident membrane of the protein (a cis interaction). ${ }^{70}$ To further examine the electrostatic interactions with negative charges on the liposome membranes, ${ }^{67,71}$ a set of experiments was performed in which the PS content on the donor proteoliposomes was varied (Figure 3.3c). When the donor proteoliposomes were neutral, namely, 0\% PS, and only the acceptor liposomes contained anionic lipids, the measured lifetime was surprisingly short ( $2.82 \pm 0.09 \mathrm{~ns}$ ) even in the absence of $\mathrm{Ca}^{2+}$ and was similar to that of the shortest set of DNAtethered liposomes $(3.3 \mathrm{~nm})$. This short distance can be explained by the nonphysiological elimination of electrostatic repulsion between the two membranes, which exists in vivo between the negatively charged synaptic vesicle and presynaptic membranes. ${ }^{10,72}$ In addition, the membranes may be pulled into close proximity if the positively charged lysine residues on the linker adhered in trans to the surfaces of acceptor liposomes. Figure 3.3c also indicates that at an intermediate level of PS (5\%), there is an equilibrium between the 7.4- and 3.3-nm configurations.

To verify that that the numbers of membrane tethering sites between donor and acceptor liposomes are comparable in the protein and DNA samples, FCS data from the acceptor channel were utilized (Figure 3.6a). The autocorrelation functions of the acceptor fluorescence fluctuations were fitted with 3D diffusion of two species (Equations 2.12 and 2.13). The $\tau_{\mathrm{D} 1}$ values were determined by measuring the diffusion of free acceptor liposomes and were typically around $1 \mathrm{~ms}$. From the best fits the fractions of the respective species, $\rho_{1}$ and $\rho_{2}$, were obtained, and the ratio of the fractions can be expressed as:

$$
\frac{\rho_{1}}{\rho_{2}}=\frac{N_{1} B_{1}^{2}}{N_{2} B_{2}^{2}}
$$

When the average number of donor liposomes in the focal volume is 1 and that of the acceptor liposomes is $N$ (e.g. 20), the formula can be rearranged to:

$$
\frac{N_{1}}{N_{2}}=\frac{\rho_{1}}{\rho_{2}} \cdot \alpha^{2}=N-\alpha
$$

with $\alpha=B_{2} / B_{1}$ representing also the number of acceptor liposomes bound to a central donor liposome under $N$ times excess. Solving Equation 3.4 yields:

$$
\alpha=\frac{-1+\sqrt{1+4 N \rho_{1} / \rho_{2}}}{2 \rho_{1} / \rho_{2}}
$$

The analysis shows that the average number of acceptor liposome tethering sites per donor liposome did not vary by $>15 \%$ throughout the samples (Table 3.1 ). 

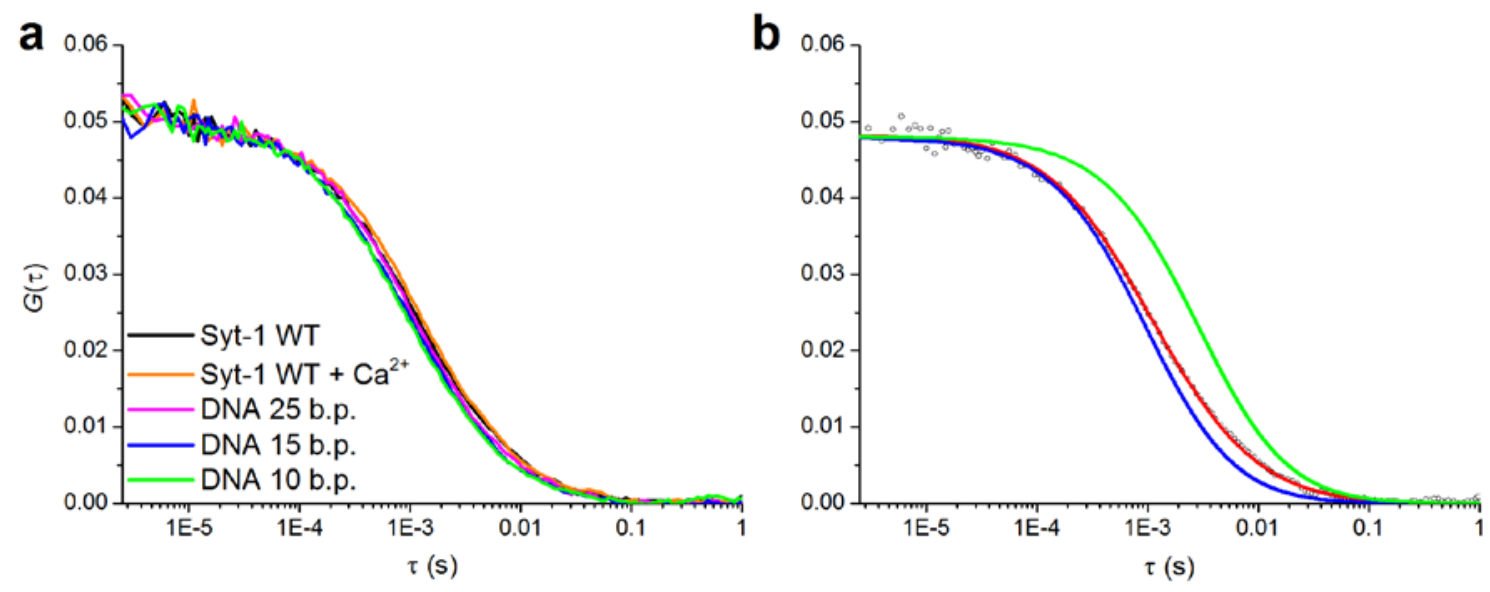

Figure 3.6. Autocorrelation curves of the acceptor signals and the 2-component fitting. (a) Normalized autocorrelation of syt-1 wild-type (WT) and DNA-tethered liposomes at the 1:20 excess ratio. (b) Representative 2-component fitting of the syt-1 sample without $\mathrm{Ca}^{2+}$. (०) The experimental data. Two diffusive components with diffusion times of $1 \mathrm{~ms}(-)$ and $3 \mathrm{~ms} \mathrm{(-)}$ were input as the initial values and $(-)$ is the final 2component fit.

Table 3.1. The number of acceptor liposomes bound to each donor liposome determined from 2-component fitting of the autocorrelation curves. Averages and standard deviations were taken from three independent repeats.

\begin{tabular}{|l|l|l|l|l|}
\hline Syt-1 (EGTA) & Syt-1 $\left(\mathbf{C a}^{2+}\right)$ & DNA 10 b.p. & DNA 15 b.p. & DNA 25 b.p. \\
\hline $2.9 \pm 0.23$ & $3.0 \pm 0.19$ & $3.3 \pm 0.10$ & $2.6 \pm 0.12$ & $2.8 \pm 0.18$ \\
\hline
\end{tabular}

\subsection{3 $\mathrm{Ca}^{2+}$-binding mutants}

To gain more insight into the $\mathrm{Ca}^{2+}$ effects, the experiments were repeated using the $\mathrm{a}^{*} \mathrm{~B}, \mathrm{Ab}^{*}$ and $\mathrm{a}^{*} \mathrm{~b}^{*} \mathrm{Ca}^{2+}$-binding mutants described in Section 2.2.1. In the mutant with a disrupted $\mathrm{C} 2 \mathrm{~B}$ domain $\left(\mathrm{Ab}^{*}\right)$, the inter-membrane distances in the absence and presence of $\mathrm{Ca}^{2+}$ were very similar to those of the wild-type protein (Figure 3.3d,g,h). On the contrary, in mutants with a disrupted C2A domain ( $\mathrm{a} * \mathrm{~B}$ and $\mathrm{a} * \mathrm{~b} *$ ), there was little or no distance shortening on addition of $\mathrm{Ca}^{2+}$, respectively (Figure 3.3e,f,h). These data support a model according to which the C2A domain is predominantly responsible for distance shortening due to $\mathrm{Ca}^{2+}$-mediated cis binding to its own membrane. ${ }^{43}$ In contrast, the C2B domain, at least under our experimental conditions, does not appear to be capable of simultaneous cis-trans binding as previously suggested. ${ }^{35,73}$ The fact that $\mathrm{a}^{*} \mathrm{~B}$ still exhibits a minor decrease of distance on $\mathrm{Ca}^{2+}$ addition may rather be 
rationalized by the $\mathrm{Ca}^{2+}$-induced binding of $\mathrm{C} 2 \mathrm{~B}$ to the acceptor membrane and the insertion of its hydrophobic residues. ${ }^{36,74}$

Figure 3.7 presents a model deduced from the current experimental findings. Initially before the $\mathrm{Ca}^{2+}$ signal arrives, the poly-lysine patch located on the C2B domain is targeted to PIP2 clusters on the presynaptic plasma membrane (Figure 3.7a,b). ${ }^{75,76}$ At this stage, full-length syt-1 maintains the two membrane bilayers at a distance of $\sim 7-8 \mathrm{~nm}$ (Figure 3.7b), possibly with the positively charged portion of the linker adhered to the host membrane. ${ }^{70}$ The $\sim 7-8 \mathrm{~nm}$ distance corresponds to that when the SNARE motifs start to assemble and form the coiled coil fourhelix bundle, ${ }^{12}$ implicating that syt- 1 holds the two membranes at this distance so that the fusion machinery is ready for fast initiation. Recently, such a pre-triggered state has also been captured in cryo-EM images which revealed that a large number of small liposomes incorporating syt-1 and synaptobrevin-2 remained docked to giant liposomes with the $t$-SNAREs at distances on the order of $\sim 10 \mathrm{~nm}$, even after extended incubation. ${ }^{77}$ The fact that removing synaptobrevin-2 or adding its soluble counterpart to inhibit the full-length SNARE assembly did not alter the overall tethering suggests the major role of syt-1 in establishing a first contact to the target membrane, upstream of SNARE nucleation. ${ }^{43,78}$

a

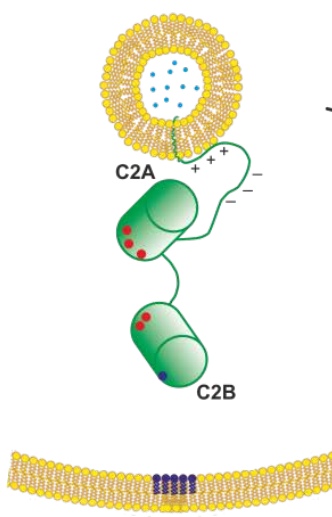

b
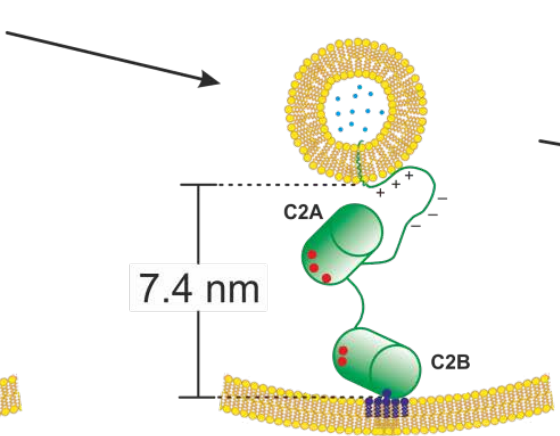

C
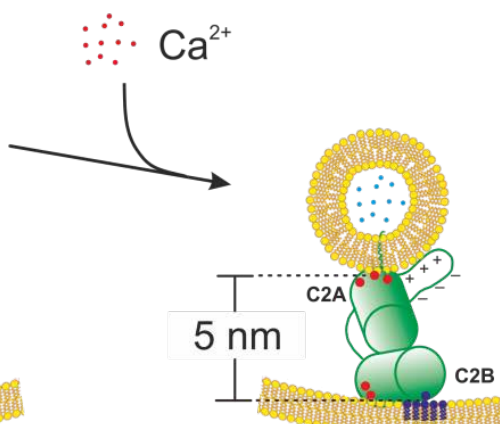

Figure 3.7. Model of full-length syt-1 binding across the synaptic vesicle and the presynaptic plasma membrane. (a) Syt-1 targets PIP2 clusters via the poly-lysine patch (blue). (b) The distance between two membranes is maintained at $\sim 7-8 \mathrm{~nm}$, as the linker is not fully stretched. (c) On $\mathrm{Ca}^{2+}$ influx, the $\mathrm{C} 2 \mathrm{~A}$ domain binds cis, reducing the distance to $5 \mathrm{~nm}$ and potentially promoting the electrostatic zippering of the linker. Both membranes contain PS. The vesicles are not drawn to scale.

Secondly, the inter-membrane gap is compressed to $\sim 5 \mathrm{~nm}$ on $\mathrm{Ca}^{2+}$ influx (Figure 3.7c). As a trans-SNARE complex spans two membranes at $\geq 4 \mathrm{~nm},{ }^{79}$ distance reduction from $\sim 7-8 \mathrm{~nm}$ to 
$\sim 5 \mathrm{~nm}$ brings the two membranes to a level at which very likely full assembly of the SNAREs is promoted, ${ }^{80}$ leading subsequently to the initiation of membrane fusion and neurotransmitter release. The membrane distance of $5 \mathrm{~nm}$ also correlates to cryo-EM (electron microscopy) observations for liposomes clustered by soluble $\mathrm{C} 2 \mathrm{AB}$ fragments and $\mathrm{Ca}^{2+},{ }^{73,81}$ in which the C2A and C2B domains bind to opposing membranes. ${ }^{82}$ Such an antiparallel conformation has been shown to be of the lowest energy using $\mathrm{C} 2 \mathrm{AB}$ from simulated annealing based on EPRderived restraints. ${ }^{83-85}$ Moreover, it corroborates new findings that alteration in the length and rigidity of the short (9-residue) linker between the C2 domains has a significant impact on evoked neurotransmitter release. ${ }^{86}$

In vivo mutation studies demonstrated that the $\mathrm{C} 2 \mathrm{~B}$ domain is indispensable for fast synchronous neurotransmitter release. ${ }^{87,88}$ In the experiments herein, initial binding of C2B to acceptor membranes is essential for the membrane distance control (Figure 3.3b). The binding was permitted to the maximum extent during incubation in EGTA buffer, in order to ensure that the differences in fluorescence decays were solely caused by $\mathrm{Ca}^{2+}$-induced distance changes. However, in the presynaptic active zone, $\mathrm{Ca}^{2+}$ binding to C2B may help to recruit previously undocked vesicles, rendering the $\mathrm{Ca}^{2+}$-dependence of the $\mathrm{C} 2 \mathrm{~B}$ mutant more severely impaired. On the other hand, the functional importance of the $\mathrm{C} 2 \mathrm{~B} \mathrm{Ca}^{2+}$-binding sites may be attributed to the ability of syt- 1 to facilitate curvature of the plasma membrane $e^{89,90}$ or interact directly with the SNAREs. ${ }^{5,85}$ The C2A domain, contrarily, plays a more important role in distance regulation. An effective decrease of the inter-membrane gap can be accomplished via binding of C2A back to the vesicle membrane and an even tighter binding of C2B to the target plasma membrane (Figure 3.7c). Notably, recently an $\sim 80 \%$ decrease in release has been demonstrated using a D229E (aspartate-to-glutamate) mutation in Drosophila at the $\mathrm{Ca}^{2+}$-coordination site of C2A, ${ }^{91}$ supporting the important function also of C2A in synchronous synaptic transmission.

\subsubsection{Modeling FRET across liposomes}

Thus far the newly developed DNA-based membrane distance ruler has been applied to measure the distance between liposomes tethered by full-length syt-1. The fact that the $5 \mathrm{~nm}$ distance obtained in $100 \mu \mathrm{M} \mathrm{Ca}^{2+}$ matched the average distances observed in cyro-EM images using soluble $\mathrm{C} 2 \mathrm{AB}$ fragments hints conversely that the ruler was well calibrated. As a supplement, an attempt was made (using Python 2.7, http://www.python.org) to model the intermembrane FRET interactions and to simulate the donor fluorescence decays.

To begin with, donor (Oregon Green 488) and acceptor (Texas Red) molecules were first distributed uniformly over the surfaces of corresponding spheres representing small unilammelar liposomes $40 \mathrm{~nm}$ in diameter (Figure 3.8). ${ }^{92}$ The number of fluorophores to be placed was decided by the product of the labeling percentage (0.5\% Oregon Green 488 or $1 \%$ 
Texas Red) and the number of lipids constituting the outer or inner leaflet. The surface area per lipid head group was taken to be $0.7 \mathrm{~nm}^{2}$ and the bilayer thickness $4 \mathrm{~nm},{ }^{93}$ amounting to 7181 and 4596 total lipids in the outer and inner leaflets, respectively.

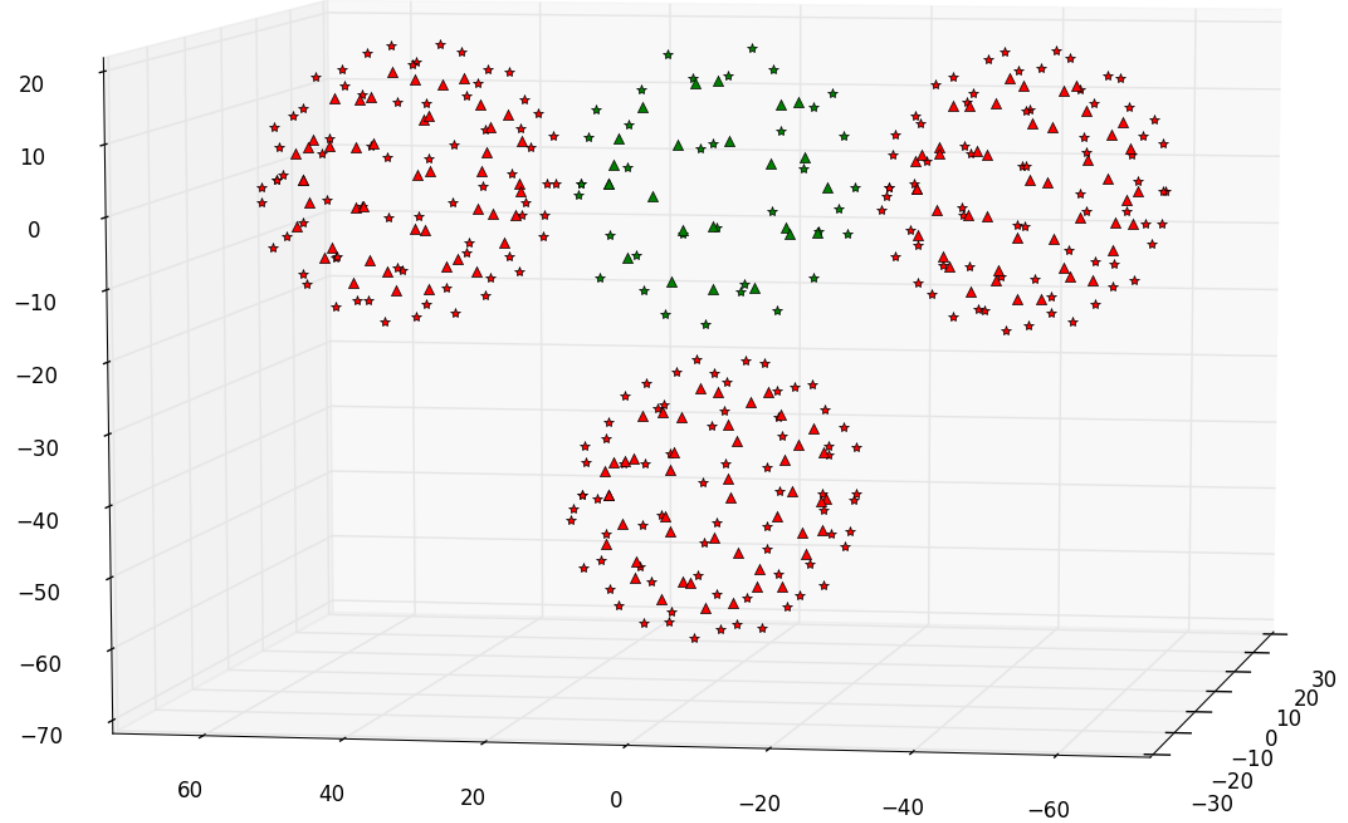

Figure 3.8. Distribution of donor (green) and acceptor (red) labels in the modeling.

$(\lesssim)$ are on the outer and $(\triangle)$ on the inner leaflets, respectively. Axes are in nm.

Throughout the modeling, the donor liposome was accompanied by three acceptor liposomes, as estimated in Section 3.1.2 with FCS. For simplicity, the transition dipole orientation of each molecule was fixed at the surface normal vector, which might have led to enhanced FRET because collinear transition dipoles have the highest $\kappa^{2}$ value of 4 (Equation 1.4 and Figure $1.2 a)$.

Next, the energy transfer rate $k_{\mathrm{ET}}$ (Equation 1.3) of a single donor to all acceptors were additive $^{94}$ and summed up to obtain the observed lifetime ( $\tau_{\mathrm{D}}$ ) of the individual donors. The $R_{0}$ value was evaluated to be $6.3 \mathrm{~nm}$, using information from the supplier (Molecular Probes) and reference $95 . \kappa^{2}$ was assumed to be 0.476 , the condition for a range of orientations which did not change during the excited state lifetime, restricted by neighboring lipid head groups. ${ }^{4}$ The individual decay functions were then convoluted with a Gaussian IRF (0.24 ns FWHM) and summed up again to generate the overall decay. The calculation of an overlap integral (Equation 2.2) was replaced with a built-in error function representing the convolution of an exponential with a Gaussian: 


$$
\exp \left[\left(\frac{B}{2 \cdot 166511 \cdot \tau_{\mathrm{D}}}\right)^{2}-\frac{t-t_{0}}{\tau_{\mathrm{D}}}\right] \cdot \frac{1}{2}\left[\operatorname{erf}\left(\frac{t-t_{0}}{B} \cdot 1.66511-\frac{B}{2 \cdot 166511 \cdot \tau_{\mathrm{D}}}\right)+1\right]
$$

where $t_{0}$ and $B$ denote the center and FWHM of the Gaussian, respectively. ${ }^{96}$

Surprisingly, the simulated decays at the three DNA spacings were much slower than the experimental results (Figure 3.9a, gray curves), which is tentatively ascribed to the slow solvent relaxation processes on membrane surfaces. In stark contrast to picosecond solvation in aqueous solutions, ${ }^{97,98}$ reorientation of water molecules around the excited donor to stabilize its redistribution of charges (Figure 3.9b) is hindered by the reorganization of lipids. In reference 99, with a combination of quantum mechanical and molecular dynamics simulations, relaxation times at the lipid/water interface was determined to be $>2$ ns (for membrane probes Prodan and Laurdan), which was comparable to the fluorescence lifetime and has recently been proven experimentally by Ernsting and coworkers (2.45 ns for Laurdan, conference paper). ${ }^{100}$ Such slow solvation kinetics should render observed decays wavelength-dependent, ${ }^{101}$ which was confirmed to be the case for Oregon Green 488 DPPE incorporated into liposomes (Figure 3.10).
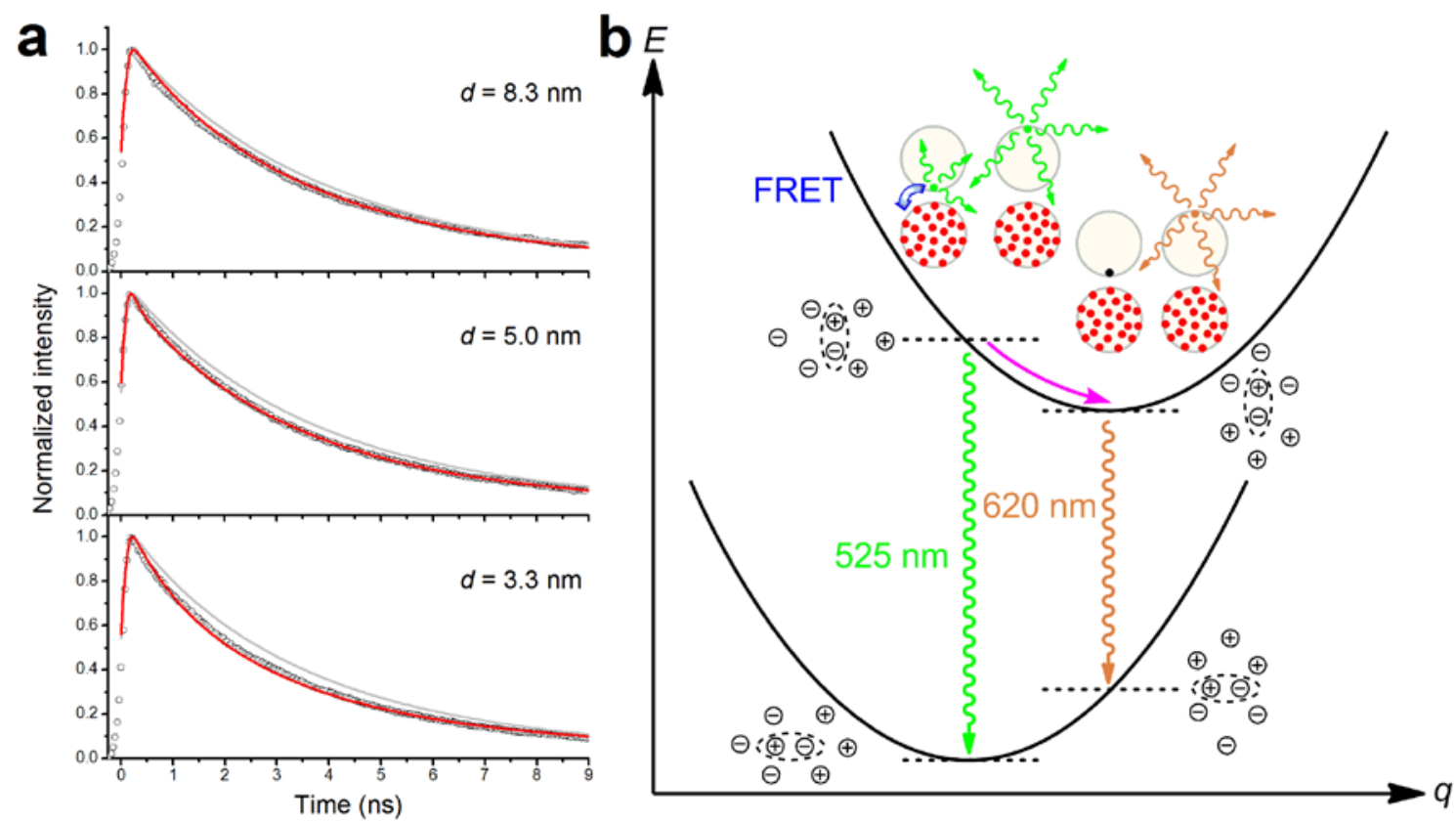

Figure 3.9. Modeling of FRET from donor to acceptor liposomes. (a) The comparison of simulated fluorescence decay curves with experimental data $(\circ) .(-)$ without and $(-)$ with the weighting factor. (b) Schematic of solvent relaxation and the resultant spectral red shift. The $y$-axis is energy and $\mathrm{x}$-axis the solvent orientation coordinate.

With the resultant spectral red shift (Figure 3.9b) at the nanosecond time scale, donors with longer lifetimes (the ones away from acceptor liposomes and thus less likely to undergo FRET) 
experience the shift and will emit a portion of photons at longer wavelengths outside of the blue-edged observation window ( $525 \pm 10 \mathrm{~nm}$, Figure 3.4). Conversely, shorter-lived donors (those facing acceptor liposomes) mostly emit within the range and their contributions to the overall decay are "amplified”. At current stage, a weighting factor chosen arbitrarily in the form of $\mathrm{e}^{-\tau \mathrm{D} / \imath \mathrm{SR}}$ (with solvent relaxation times $\tau_{\mathrm{SR}}$ ranging from 1.6 to $2.7 \mathrm{~ns}$ ) is multiplied to each donor decay function before summation, thereby attenuating the contributions of longer-lived donors or, equivalently, amplifying those of the shorter-lived ones (Figure 3.9a, red curves).
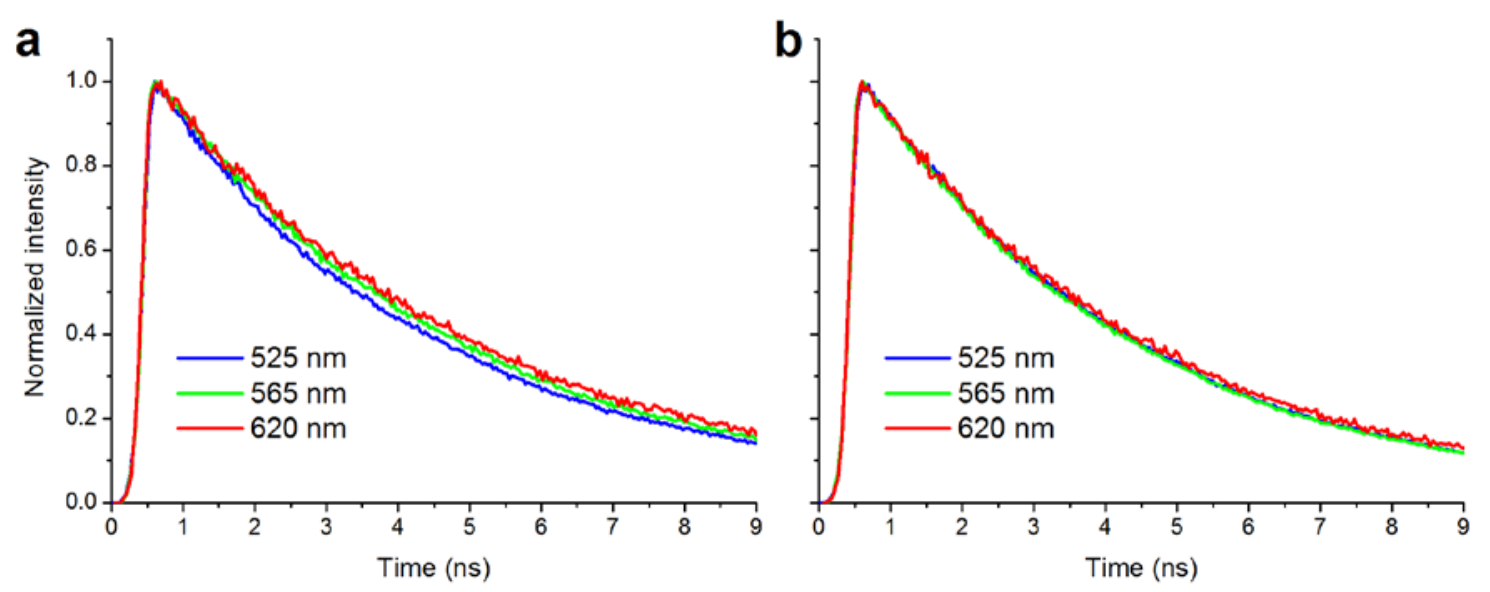

Figure 3.10. Influence of solvent relaxation on the fluorescence decays measured at different wavelengths. (a) For Oregon Green 488 DPPE, solvent relaxation at the lipid/water interface is a nanosecond process, and the measured decay is wavelengthdependent. (b) Free Oregon Green 488 molecules in aqueous buffer solutions experience picosecond solvation which cannot be detected with TCSPC.

Finally, it is worthy to note that deformation of the tethering sites might deviate the measured decays from the theoretical model. Nonetheless, cryo-EM images have clearly revealed that small unilamellar liposomes, owing to the high membrane curvature, still preserved the spherical shape when tethered to giant liposomes (by syt-1 and/or the SNAREs). ${ }^{77}$ On the contrary, protrusions were seen on the less curved giant liposome surfaces. 


\subsection{Asymmetrically labeled liposomes as a new tool to study membrane fusion}

Previously in Chapter 3.1, FRET across two membrane affected the donor lifetime $\left(\tau_{\mathrm{D}}\right)$, which in turn governed the donor decay. In this part, instead, the pre-exponential amplitudes $A_{\mathrm{i}}$ (Equation 2.1) of the acceptors serve as the indicator of FRET. More FRET interactions give rise to more excited acceptors and consequently larger amplitudes.

The goal of this project is to develop a bulk fluorescence assay with which fusion of the outer and inner leaflets of two membranes can be monitored simultaneously, so that the hemifusion intermediate (Figure 1.4) may be pinpointed in a single experiment. Even with the advancement of single-molecule methods, the delay between fusion of the two leaflets has exclusively been inferred indirectly from transient intermediate FRET states ${ }^{19}$ or mixing of the contents enclosed within the liposomes. ${ }^{20,21}$ The design principle herein is to label the leaflets with two different acceptor dyes which produce distinct FRET signals after fusion with homogeneously labeled donor liposomes.

As a proof of concept, the fluorescent dyes of choice were NBD and Oregon Green 488 being the acceptors and Marina Blue as the donor (all spectra displayed in Figure 3.11a). Among them, NBD was critical because its green fluorescent charge-transfer band could easily be eliminated by reduction with sodium dithionite $\mathrm{Na}_{2} \mathrm{~S}_{2} \mathrm{O}_{4}$ (Section 2.2.3), ${ }^{59}$ rendering the outer leaflet unlabeled.
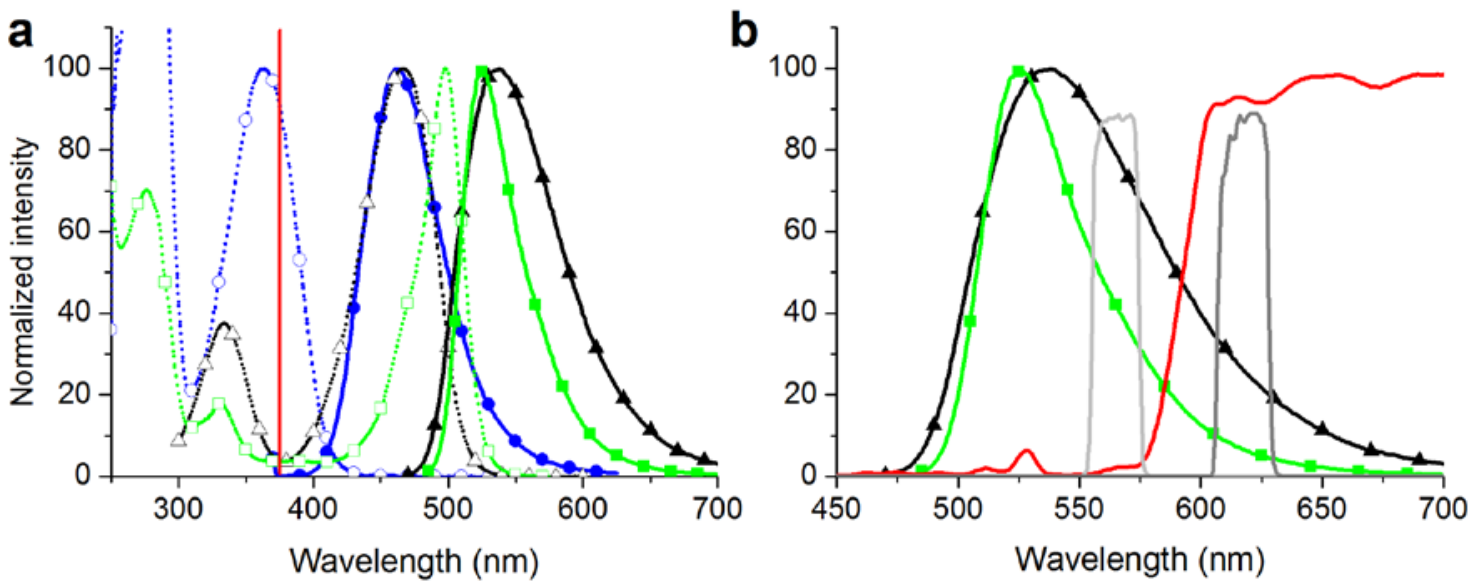

Figure 3.11. Spectra of the one donor-two acceptor system. $(-)$ Marina Blue, $(-)$ NBD and ( - ) Oregon Green 488. (a) Dotted lines with open symbols are the absorption and solid lines with closed symbols the fluorescence emission. The red vertical line marks the excitation wavelength $(375 \mathrm{~nm})$. (b) Spectra of the acceptor emissions together with the dichroic mirror 590DCXR $(-)$ and band pass filters D565/20m and D620/20m ( - and - ). 
Secondly, Marina Blue exhibits decent spectral overlap with NBD and was therefore a good candidate as the donor. Oregon Green 488 was chosen lastly as another acceptor to be added onto the outer leaflet via the reaction with thiol-functionalized lipids (Figures 2.6 and 2.10). Overlap of the NBD and Oregon Green 488 emission spectra implicates that the signals cannot be clearly discriminated using steady-state spectroscopy. Rather, the difference in fluorescence lifetime was utilized. Intrinsic lifetimes of Oregon Green 488 and NBD are approximately 4 and 7 ns, respectively (Figure 3.12a,b), which shall not be altered by FRET (Equation 1.9). Lifetime measured at acceptor wavelengths for hemifused liposomes are expected to be shorter than that of either the fully fused or unfused ones, since FRET excites predominantly Oregon Green 488 on the outer leaflet.
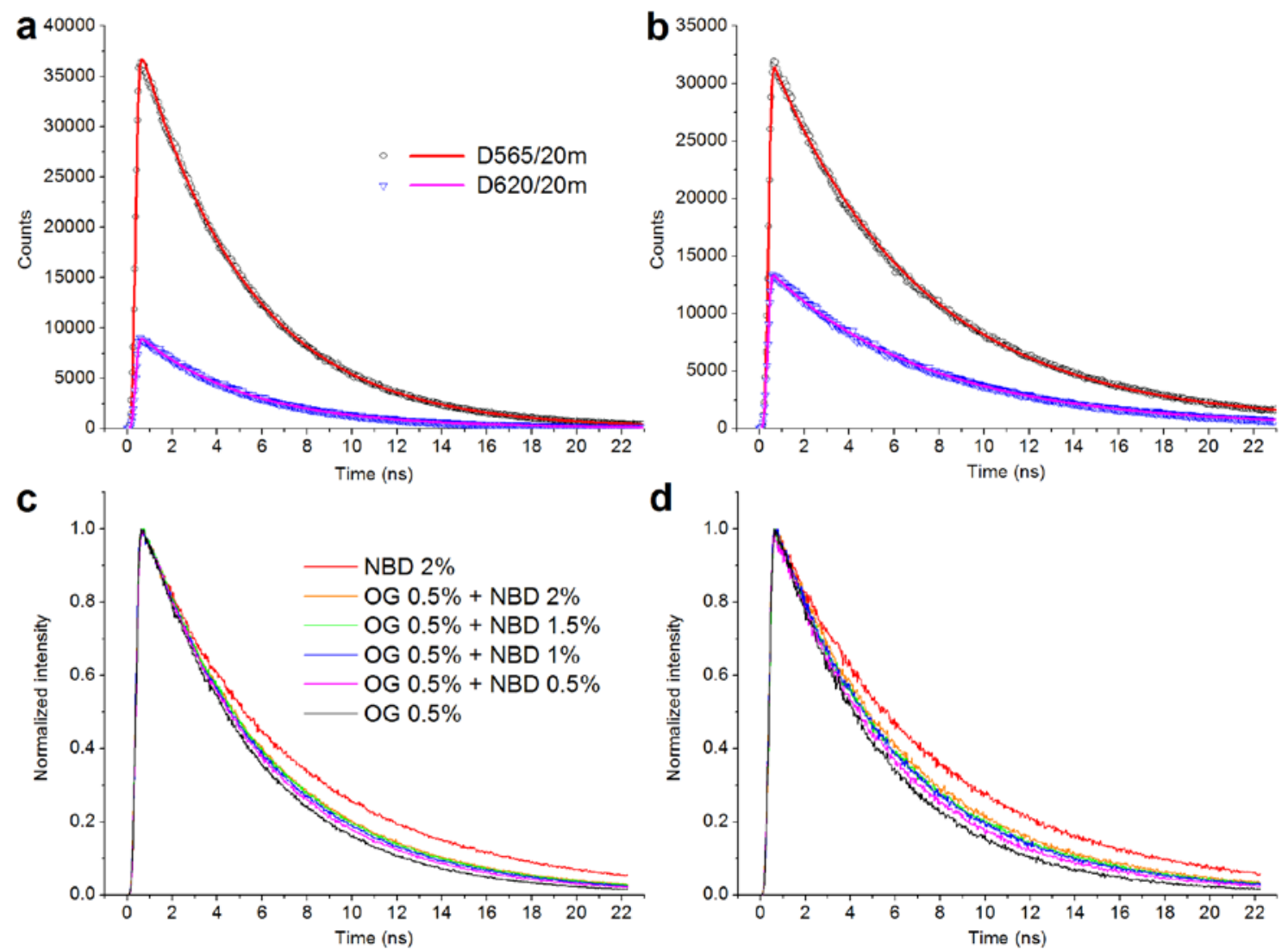

Figure 3.12. Fluorescence decays of liposomes with various labeling percentages, measured at two wavelengths. (a) $0.5 \%$ Oregon Green 488. (b) $2 \%$ NBD. The symbols represent experimental data, whereas the lines are reconvolution fits. Difference in intensity ratios between the two channels is visible. (c,d) Experimental decay curves for the specified mixtures (towards less NBD) measured at $565 \mathrm{~nm}(\mathbf{c})$ and $620 \mathrm{~nm}(\mathbf{d})$. The contribution of NBD is higher at longer wavelengths. 


\subsubsection{Differentiating two acceptor labels with the fluorescence lifetime}

To confirm that the 2-3 ns difference in fluorescence lifetime was sufficient for differentiating the two acceptor labels, liposomes including various amounts of Oregon Green 488 DPPE and NBD DPPE were first measured (Figures 3.12c,d and 3.13). With 375 nm excitation, a 1:4 Oregon Green 488-to-NBD ratio ( 0.5 vs. $2 \%$ ) yielded a decay curve roughly in the middle of the two intrinsic decays. Apparently, the decay curves were in order when either fluorophore was lessened. Nonetheless, ratiometric measurements at two wavelengths, 565 and $620 \mathrm{~nm}$, were conducted to ensure that the amplitudes retrieved from 2-component fits (Equation 3.7) were unambiguous.

$$
F(t)=A_{\mathrm{OG}} e^{-t / \tau_{\mathrm{OG}}}+A_{\mathrm{NBD}} e^{-t / \tau_{\mathrm{NBD}}}
$$

By fitting the four curves in Figure 3.12a,b, six parameters for 2-component fitting could be deduced: $\tau_{\mathrm{OG}}$ at $565 \mathrm{~nm}(4.8 \mathrm{~ns})$ and $620 \mathrm{~nm}(4.8 \mathrm{~ns}) ; \tau_{\mathrm{NBD}}$ at $565 \mathrm{~nm}(6.7 \mathrm{~ns})$ and $620 \mathrm{~nm}(7.0$ ns); amplitude ratios $A_{620} / A_{565}$ for Oregon Green 488 (0.24) and NBD (0.42). With the six parameters fixed, a fitting routine (written with Python 2.7, http://www.python.org) was then used to derive the best-fitting pair of functions, akin to global analysis in dealing with timeresolved spectra. ${ }^{96,102}$ The resultant amplitudes were compared with those obtained directly from the measurement software SymPhoTime (version 5.3, PicoQuant), and the two fitting methods returned similar values. Namely, individual best fits at either wavelength represented the best global solution and vice versa. Summarized in Table 3.2 are the relative amplitudes $\left(A_{\mathrm{NBD}} / A_{\mathrm{OG}}\right.$ or $\left.A_{\mathrm{OG}} / A_{\mathrm{NBD}}\right)$ at $565 \mathrm{~nm}$, which are consistent with the sample compositions.
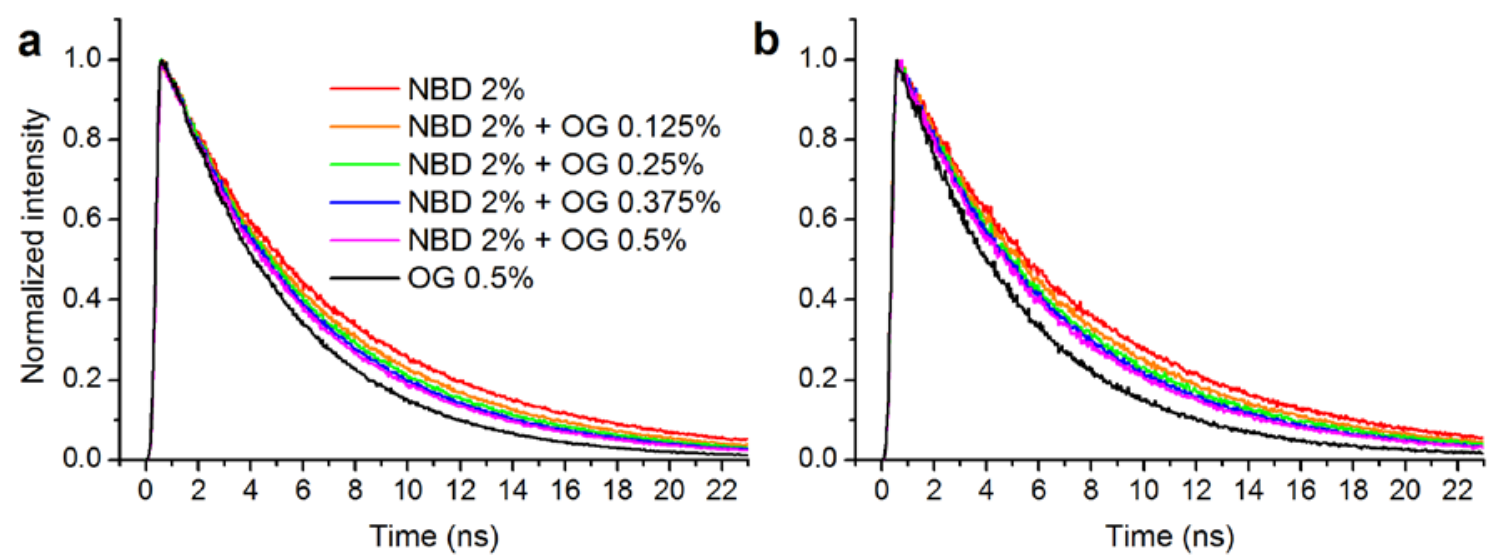

Figure 3.13. Fluorescence decays of a second set of mixtures towards less Oregon

Green 488. Measured at $565 \mathrm{~nm}$ (a) and $620 \mathrm{~nm}$ (b). 
Table 3.2. Relative amplitudes at $565 \mathrm{~nm}$ from two sets of measurements. Rel designates the comparison with respect to the value for NBD $2 \%$ and OG $0.5 \%$.

\begin{tabular}{|c|c|c|c|c|c|}
\hline NBD (\%) & OG (\%) & $\boldsymbol{A}_{\mathrm{NBD}} / \boldsymbol{A}_{\mathrm{OG}}$ & Rel & $A_{\mathrm{OG}} / A_{\mathrm{NBD}}$ & Rel \\
\hline 2 & 0.5 & 0.76 & 1 & 1.55 & 1 \\
\hline 1.5 & 0.5 & 0.58 & 0.77 & - & - \\
\hline 1 & 0.5 & 0.41 & 0.54 & - & - \\
\hline 0.5 & 0.5 & 0.22 & 0.29 & - & - \\
\hline 2 & 0.375 & - & - & 1.09 & 0.70 \\
\hline 2 & 0.25 & - & - & 0.72 & 0.46 \\
\hline 2 & 0.125 & - & - & 0.36 & 0.23 \\
\hline
\end{tabular}

Next, 1\% of the FRET donor Marina Blue was incorporated into liposomes along with $0.25 \%$ Oregon Green 488 or $0.25 \%$ Oregon Green 488 plus 1\% NBD, mimicking the effect of 1-to-1 hemifusion or full fusion between donor liposomes labeled with 2\% Marina Blue and acceptor liposomes with $0.5 \%$ Oregon Green 488 plus 2\% NBD. The two types of liposomes were then mixed at various ratios to simulate the population evolution from entirely hemifused to entirely fully fused. The recorded decay curves again complied with the trend in preparation (Figure 3.14).
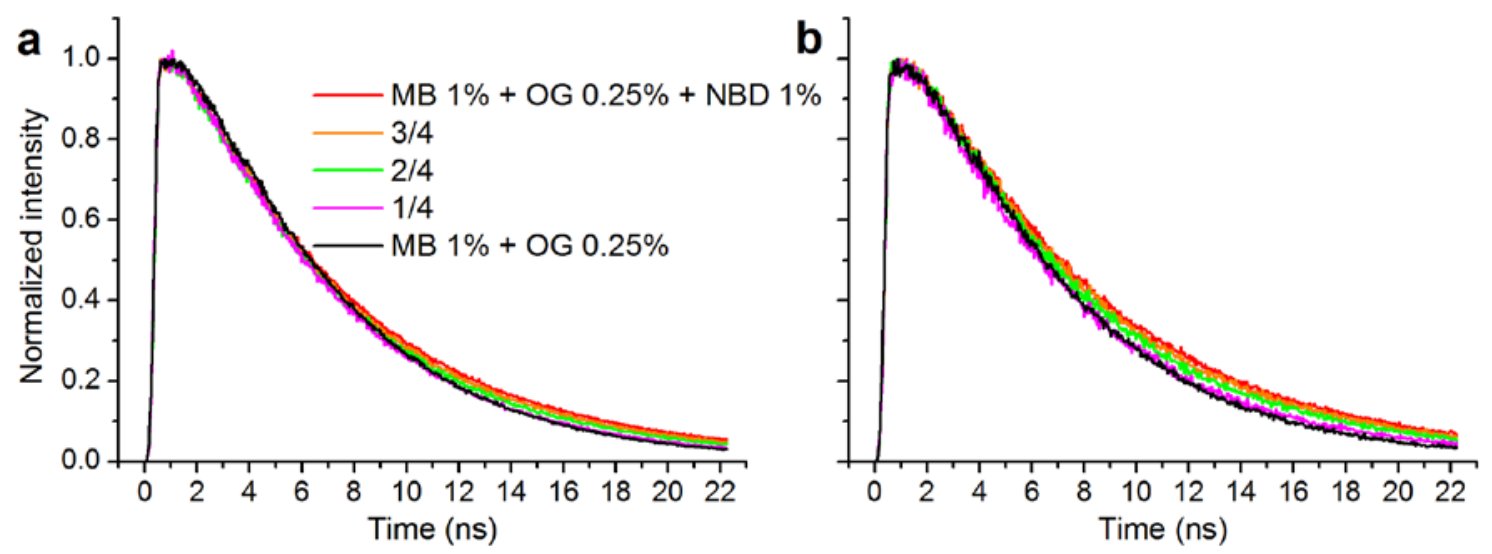

Figure 3.14. Fluorescence decays of liposome mixtures mimicking the progression from hemifusion to full fusion. Measured at acceptor wavelengths of $565 \mathrm{~nm}$ (a) and $620 \mathrm{~nm}(\mathrm{~b})$. The three fractions designate the proportion of liposomes carrying 1\% Marina Blue, $0.25 \%$ Oregon Green 488 and 1\% NBD. 
Also visible in Figure 3.14 is the rounded shape at the beginning of the decays (compared with those in Figures 3.12 and 3.13), which is attributable to the rising component with a negative pre-exponential amplitude in the precursor-successor relationship (Equation 1.9). In a separate series of experiments, labeling percentages of Marina Blue and either Oregon Green 488 or NBD were successively diluted, deviating the lifetime curves more from normal decays (Figure 3.15). This could be accounted for by the slowing down of FRET rate while the fluorophores became sparser. The rise time constants $\left(\tau_{\text {rise }}\right)$ and the fractions of their amplitudes $\left(A_{\text {rise }}\right)$ to the sum of all amplitudes (in absolute values, $\left.\left|A_{\mathrm{i}}\right|\right)^{103}$ from the fits to Figure 3.15 are listed in Table 3.3. The fractions are smaller than 0.5 as predicted from Equation 1.9, mainly because a portion of the acceptors were excited directly rather than via FRET.
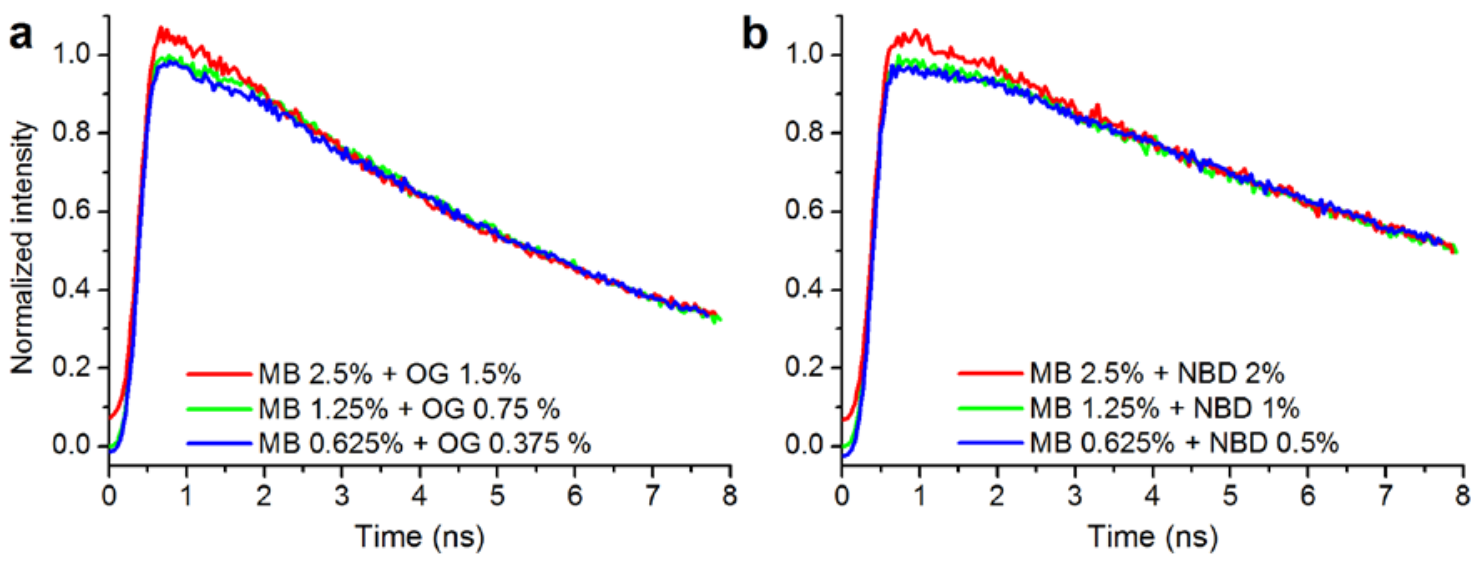

Figure 3.15. The deviation of acceptor signals at early times varied with labeling percentages. Measured at $565 \mathrm{~nm}$ with the acceptor being Oregon Green 488 (a) or NBD (b). The curves were shifted vertically and matched to the tails for comparison.

Table 3.3. Rise time constants and associated amplitudes from the fits to Figure 3.15.

\begin{tabular}{|lllllll|l|l|}
\hline & & & & & & $\tau_{\text {rise }}(\mathbf{n s})$ & $A_{\text {rise }} / \Sigma\left|A_{\mathbf{i}}\right|$ \\
\hline MB & 2.5 & $\%$ & OG & 1.5 & $\%$ & 0.64 & -0.17 \\
\hline MB & 1.25 & $\%$ & OG & 0.75 & $\%$ & 1.24 & -0.24 \\
\hline MB & 0.625 & $\%$ & OG & 0.375 & $\%$ & 1.56 & -0.25 \\
\hline MB & 2.5 & $\%$ & NBD & 2 & $\%$ & 0.44 & -0.12 \\
\hline MB & 1.25 & $\%$ & NBD & 1 & $\%$ & 1.05 & -0.17 \\
\hline MB & 0.625 & $\%$ & NBD & 0.5 & $\%$ & 1.53 & -0.19 \\
\hline
\end{tabular}




\subsubsection{Two-step labeling}

After establishing the FRET system comprising of one donor and two acceptors, the two chemical reactions, illustrated in Figure 2.10, to prepare asymmetrically labeled acceptor liposomes were carried out and conveniently characterized with FCS (Figure 3.16).
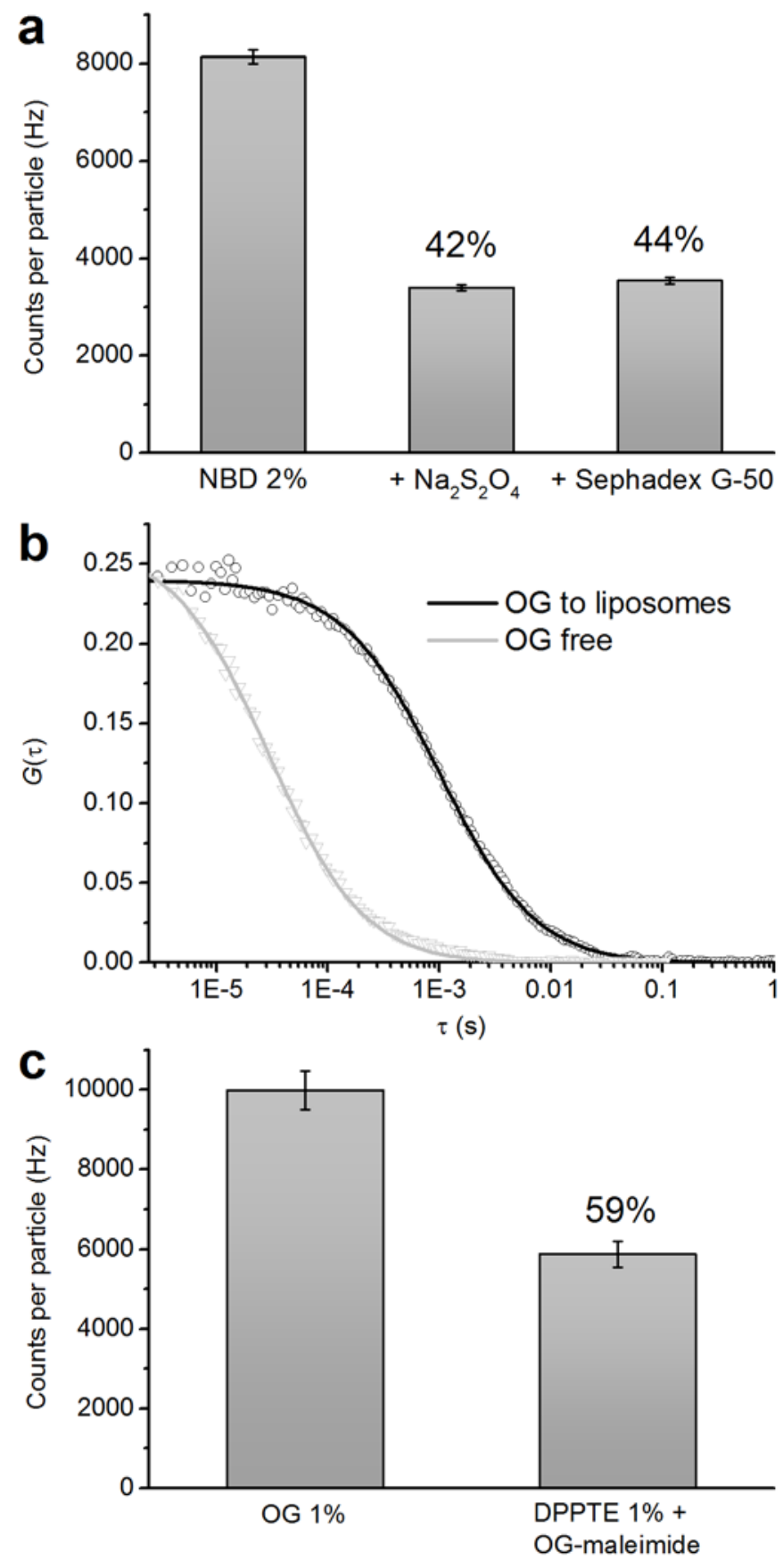

Figure 3.16. Characterization of the two reactions with FCS. (a) Reduction by dithionite at the outer leaflet of $2 \%$ NBD liposomes decreased the brightness to $\sim 40 \%$. Excitation wavelength: $800 \mathrm{~nm}$ (two-photon). Emission filter: HQ535/50m. The average values and s.d. were obtained from 4 measurements: $0,1,2$ and 3 hours after the reaction. 
(b,c) Addition of Oregon Green 488 maleimide to the outer leaflet of liposomes carrying $1 \%$ DPPTE yielded liposomes $\sim 60 \%$ as bright as liposomes labeled directly with $1 \%$ Oregon Green 488 DPPE. Excitation wavelength: $840 \mathrm{~nm}$ (two-photon). Emission filter: HQ535/50m. The average and marginal values were the outcome of 2 measurements.

The characterization was based on the brightness of liposomes ( $B_{\mathrm{i}}$ in Equation 2.12), which is equivalent to the fluorescence signal (in $\mathrm{Hz}$ ) divided by the average number of liposomes in the focal volume ( $<N>$ in Equation 2.9). Meanwhile, $<N>$ is equal to the inverse of autocorrelation function at zero time-shift $(\tau=0)$.

First, depletion of NBD on the outer leaflet was tested by incubating equal volumes of $2 \%$ NBD liposomes with $200 \mathrm{mM} \mathrm{Na}_{2} \mathrm{~S}_{2} \mathrm{O}_{4}$ for 5 minutes at room temperature. ${ }^{57}$ Half of the reacted liposomes were subjected to a second Sephadex G-50 Superfine column to remove excess $\mathrm{S}_{2} \mathrm{O}_{4}{ }^{2-},{ }^{104}$ and in this step the liposomes were diluted by $\sim 5-10$ times. The brightness of unreacted, reacted as well as reacted and purified liposomes was measured 0, 1, 2 and 3 hours after the reaction. Comparison of the three samples (Figure 3.16a) demonstrates that the reduction decreased the brightness to $\sim 40 \%$, scaling precisely with the fraction of lipids in the inner leaflet. Resemblance of the latter two samples implies that few $\mathrm{S}_{2} \mathrm{O}_{4}{ }^{2-}$ ions permeated into the liposomes to quench the inner NBD molecules.

Second, liposomes bearing 1\% thiol-functionalized DPPTE (Figure 2.6) and additionally 1.25\% Marina Blue (thereby permitting on-column tracking with fluorescence) were reacted with $54 \mathrm{nmol}$ of Oregon Green 488 maleimide powder for 2 hours at room temperature. Following removal of the remaining maleimide, also with a Sephadex G-50 Superfine column, ${ }^{58,67}$ FCS measurements affirmed that Oregon Green 488 fluorophores were successfully attached to the liposomes with $\sim 1$ ms diffusion time (black curve in Figure 3.16b). The brightness of such liposomes was $\sim 60 \%$ of that of the liposomes labeled directly with $1 \%$ Oregon Green 488 DPPE (Figure 3.16c), which coincided with the fraction of lipids in the outer leaflet. Under the experimental conditions of two-photon excitation at $840 \mathrm{~nm}$ and fluorescence collection at $535 \pm 25 \mathrm{~nm}$, liposomes carrying 1.25\% Marina Blue alone did not afford resolvable FCS traces.

Subsequently, the two reactions were combined, as detailed in Section 2.2.3, to produce liposomes labeled externally with 0.5\% Oregon Green 488 and internally with 2\% NBD.

\subsubsection{The rapid transition through hemifusion}

To reconstitute SNARE-mediated membrane fusion, synaptobrevin-2 was incorporated with a 1:500 protein-to-lipid molar ratio into acceptor liposomes composed of 2\% NBD DPPE, 0.5\% 
DPPTE, 20\% DOPE, 10\% DOPS, 10\% cholesterol and 57.5\% DOPC, which then underwent the two-step reaction in Section 2.2.3. In the meantime, the $\Delta \mathrm{N}$ complex (Section 2.2.1) was reconstituted also with a 1:500 ratio into donor liposomes consisting of 2\% Marina Blue DPPE, 20\% DOPE, 10\% DOPS, 10\% cholesterol and 58\% DOPC. The donor and acceptor liposomes, after diluted 25 and 2.5 times, respectively, were injected through separate inlets into the microfluidic channel whose design was displayed in Figure 2.11. Fluorescence lifetime of the asymmetrically labeled acceptor liposomes was measured at horizontal segments of the channel, which signified different time points after the liposomes had been thoroughly mixed.
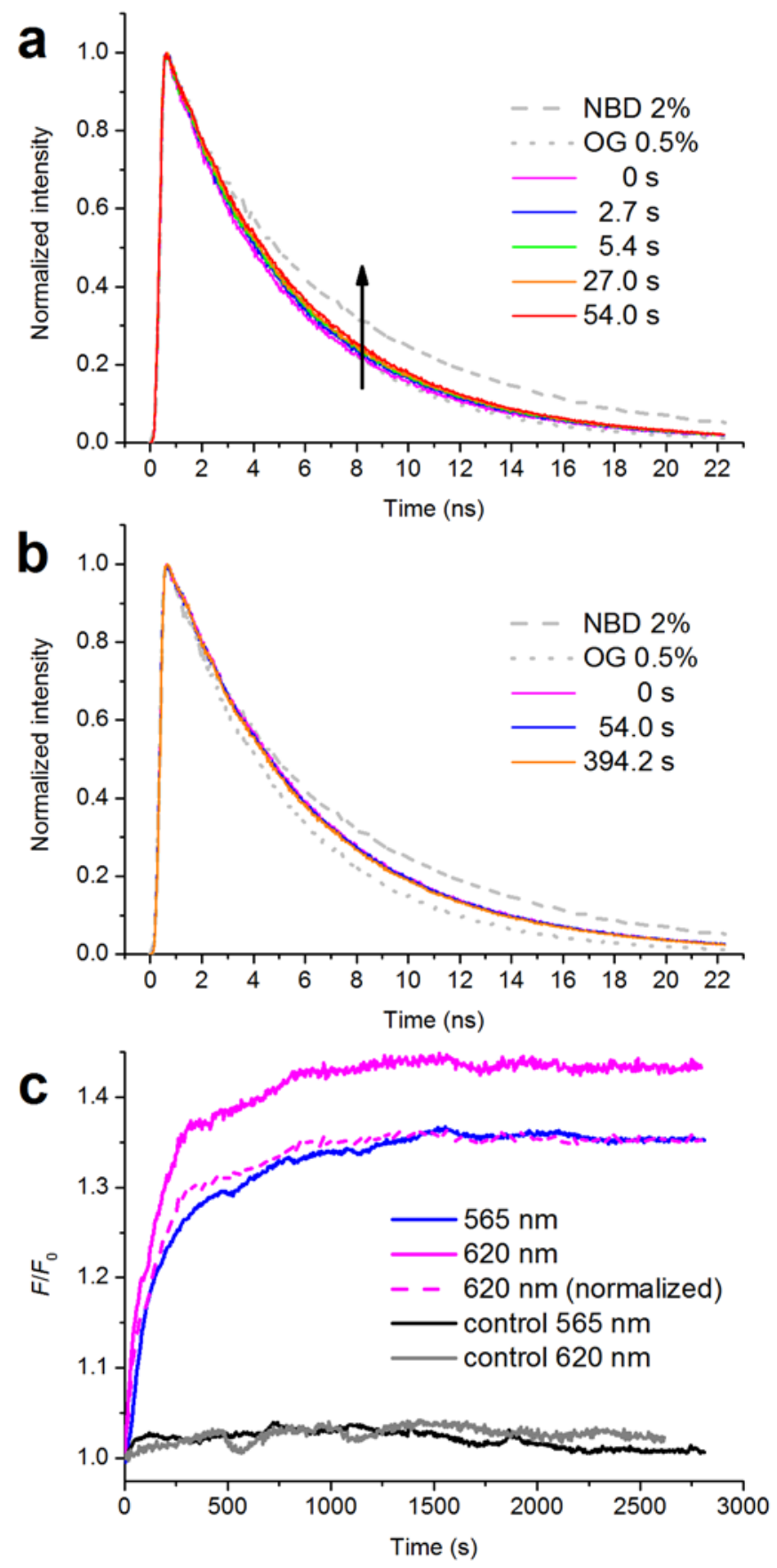
Figure 3.17. SNARE-mediated membrane fusion observed in a microfluidic channel showed faster kinetics than in a cuvette. (a) Representative fusion experiment monitored in a microfluidic channel via TCSPC at the acceptor wavelength of $565 \mathrm{~nm}$. The lifetime curves converged after a reaction time of 54 seconds. (b) There was no change in fluorescence lifetime when the $\Delta \mathrm{N}$ complex was excluded from the reaction, as was the case in the absence of synaptobrevin-2 (not shown). (c) Fusion reaction monitored with conventional time-course measurements using a fluorometer. The signals reached a plateau at 1500 seconds. Excitation wavelength: $375 \pm 2.5 \mathrm{~nm}$; emission bandwidths: $10 \mathrm{~nm}$.

The advantages of measuring in a microfluidic channel include (i) minimal sample consumption (20 $\mu \mathrm{L} / \mathrm{hr}$ ), (ii) rapid and thorough mixing in the small confined volume and (iii) enabling of prolonged and repetitive measurements at any given time point, as opposed to "single-shot" time-course measurements using a fluorometer or the stopped-flow technique (e.g., in references 36 and 74).

Shown in Figure 3.17a is a typical fusion experiment. Initially, the decay was faster and approached that of the $0.5 \%$ Oregon Green 488 liposomes. The curves were shifted subsequently towards longer lifetimes until 54 seconds and remained unchanged afterwards. This suggested a transient buildup of hemifused liposomes (in which only the external Oregon Green 488 labels were excited by FRET) at early times, which diminished as the reaction progressed efficiently to full fusion. The trend of increasing lifetime was found in all experiments. Nevertheless, duration of the increase varied from experiment to experiment, potentially due to variations in the preparation of asymmetrically labeled liposomes or fabrication of the channels. ${ }^{23,24}$ For instance, with the flow rate being constant, variations in channel height affects the timing accuracy. As control, either the $\Delta \mathrm{N}$ complex or synaptobrevin2 was excluded from the reaction, and in neither case was there a substantial change in fluorescence lifetime (Figure 3.17b). The signals originated from direct excitation of the acceptor liposomes.

Lastly, the same reaction mixtures were examined with a fluorometer (FluoroMax-2, Horiba Scientific). The acceptor fluorescence intensity at both 565 and $620 \mathrm{~nm}$ reached a plateau at approximately 1500 seconds after introduction of the donor liposomes using a pipette (Figure 3.17c). The observed kinetics was roughly 30 times slower than that in the microfluidic channel, likely due to slow mixing in the four orders of magnitude larger reaction volume ( $240 \mu \mathrm{L}$ in total). Omitting the SNARE protein on either type of liposomes again abolished fusion. Owing to spectral overlap of the two acceptors (Figure 3.11), formation of the hemifusion intermediate could not be inferred from such steady-state measurements. 
In conclusion, by integrating microfluidic channels and fluorescence lifetime measurements with proof-of-concept asymmetrically labeled liposomes, prepared from commercially available reagents, it was demonstrated that the hemifusion state constitutes a short-lived intermediate in the course of SNARE-mediated membrane fusion. Furthermore, the observed fusion kinetics can be accelerated by scaling down the overall reaction volume, emulating the conditions at miniscule presynaptic terminals. ${ }^{105,106}$ 


\section{OUTLOOK}

\subsection{Refining the membrane distance ruler}

In Chapter 3.1, a fluorescence lifetime based inter-membrane distance ruler was developed using membrane-anchored DNAs of various lengths as calibration standards. It has been successfully employed to elucidate quantitatively for the first time the distance regulation function of synaptotagmin- 1 , the $\mathrm{Ca}^{2+}$ sensor and trigger in synaptic transmission. Aside from relevant mechanisms in neuronal exocytosis, the ruler may be applied to investigate distance regulatory properties of the key components in processes ranging from intracellular membrane trafficking, viral fusion to cell-cell fusion. ${ }^{11,107,108}$

On the other hand, the theoretical model presented in Section 3.1.4 should be refined, so that (i) the fluorescence decay of the FRET donor at any defined inter-membrane distance (e.g., set by dsDNA) can be predicted and (ii) the actual distance may directly be deduced from the measured lifetime. A glitch in the current model is that the transition dipole orientations are fixed at the surface normal vectors, while in reality they explore a certain range of angles or may be tangent to the liposome surface. Further, the Förster distance $R_{0}$ is no longer constant in time when any spectral shift, which influences the spectral overlap between the donor emission and acceptor absorption, is considered.

Most importantly, the weighting factor that emphasizes the contributions of short-lived donors at the blue edge of the fluorescence spectrum should be experimentally resolved. Using broadband fluorescence upconversion spectroscopy to study the dynamic Stokes shift, ${ }^{109,110}$ Ernsting and coworkers (Humboldt University, Berlin) have confirmed with liposomeembedded Laurdan probes the slow (2.45 ns) solvent relaxation at lipid/water interfaces, ${ }^{100}$ as 
computed in reference 99. Likewise, the solvation dynamics and time-resolved emission spectra of Oregon Green 488 DPPE can be measured for the formulation of a proper weighting function.

\subsection{Optimal design of FRET from one donor to two acceptors}

In Chapter 3.2, a first example of asymmetrically labeled liposomes was demonstrated, and the inner and outer labels could be distinguished by their distinct fluorescence lifetimes. With these liposomes, a FRET system from one donor to two acceptors was created to probe the transient hemifusion state in the exocytosis of synaptic vesicles.

The readily available Oregon Green 488 fluorophore (with the maleimide functional group) was chosen in this proof of concept based on its spectral overlap with NBD, the acceptor that was essential because of the well-documented reduction reaction with dithionite ions. However, the 2-3 ns difference in lifetime between the two acceptors was far from desirable to warrant reliable fitting under all experimental conditions. One way to enlarge the difference in lifetime so as to improve the fitting is to add to the outer leaflet second- or third-row transition metal complexes whose phosphorescence decays at $\geq 100 \mathrm{~ns}^{111-113}$

Alternatively, if the external acceptor emits at longer wavelengths, the two acceptors can be differentiated more easily, even via steady-state measurements. Two approaches are envisioned. The first is to use fluorophores undergoing excited-state proton transfer, which leads to redshifted tautomer emission bands. ${ }^{114,115}$ Secondly, by using a covalently linked dyad, ${ }^{116,117}$ (e.g., by linking Texas Red to Oregon Green 488), excitation energy deposited to the donor (Marina Blue) should be efficiently relayed to the second acceptor (Marina Blue-Oregon Green 488Texas Red) upon fusion of the outer leaflet. If the APD detectors are replaced with a spectrograph (a CCD camera plus a grating), the NBD and red-shifted acceptor emissions may be recorded simultaneously with the donor fluorescence and even the scattered light, which can serve as an internal standard to calibrate for intensity fluctuations.

If two-color labels are incorporated, the applicability of asymmetrically labeled liposomes is no longer limited to in vitro studies of membrane fusion using FRET. They may be injected into cells to track the membrane recycling at different organelles ${ }^{118,119}$ and see whether lipids in the inner and outer leaflets have the same fate. Moreover, after fusing with compartmental or plasma membranes, the different diffusion behavior of lipid probes in the inner and outer leaflets can be monitored with two-color FCS. ${ }^{120,121}$ 


\section{REFERENCES}

(1) Kasha, M. Characterization of electronic transitions in complex molecules. Discuss. Faraday. Soc. 1950, 9, 14-19.

(2) Mortimer, R. G. Spectroscopy and Photochemistry. In Physical Chemistry (Second Edition); Academic Press: Burlington, 2000; pp 751-815.

(3) Takeuchi, S.; Tahara, T. The answer to concerted versus step-wise controversy for the double proton transfer mechanism of 7-azaindole dimer in solution. Proc. Natl. Acad. Sci. USA 2007, 104, 5285-5290.

(4) Lakowicz, J. R. Energy Transfer. In Principles of Fluorescence Spectroscopy; 3 ed.; Springer US, 2006; pp 443-475.

(5) Jahn, R.; Fasshauer, D. Molecular machines governing exocytosis of synaptic vesicles. Nature 2012, 490, 201-207.

(6) Jahn, R.; Lang, T.; Südhof, T. C. Membrane fusion. Cell 2003, 112, 519-533.

(7) Chernomordik, L. V.; Kozlov, M. M. Protein-lipid interplay in fusion and fission of biological membranes. Annu. Rev. Biochem. 2003, 72, 175-207.

(8) Aeffner, S.; Reusch, T.; Weinhausen, B.; Salditt, T. Energetics of stalk intermediates in membrane fusion are controlled by lipid composition. Proc. Natl. Acad. Sci. USA 2012, 109, E1609-E1618.

(9) Fasshauer, D.; Sutton, R. B.; Brunger, A. T.; Jahn, R. Conserved structural features of the synaptic fusion complex: SNARE proteins reclassified as Q- and R-SNAREs. Proc. Natl. Acad. Sci. USA 1998, 95, 15781-15786. 
(10) Takamori, S.; Holt, M.; Stenius, K.; Lemke, E. A.; Gronborg, M.; Riedel, D.; Urlaub, H.; Schenck, S.; Brugger, B.; Ringler, P.; Muller, S. A.; Rammner, B.; Grater, F.; Hub, J. S.; De Groot, B. L.; Mieskes, G.; Moriyama, Y.; Klingauf, J.; Grubmüller, H.; Heuser, J.; Wieland, F.; Jahn, R. Molecular anatomy of a trafficking organelle. Cell 2006, 127, 831-846.

(11) Jahn, R.; Scheller, R. H. SNAREs - engines for membrane fusion. Nat. Rev. Mol. Cell Biol. 2006, 7, 631-643.

(12) Li, F.; Pincet, F.; Perez, E.; Eng, W. S.; Melia, T. J.; Rothman, J. E.; Tareste, D. Energetics and dynamics of SNAREpin folding across lipid bilayers. Nat. Struct. Mol. Biol. 2007, 14, 890-896.

(13) Stein, A.; Weber, G.; Wahl, M. C.; Jahn, R. Helical extension of the neuronal SNARE complex into the membrane. Nature 2009, 460, 525-528.

(14) Sutton, R. B.; Fasshauer, D.; Jahn, R.; Brunger, A. T. Crystal structure of a SNARE complex involved in synaptic exocytosis at $2.4 \AA$ resolution. Nature 1998, 395, 347-353.

(15) Weber, T.; Zemelman, B. V.; McNew, J. A.; Westermann, B.; Gmachl, M.; Parlati, F.; Söllner, T. H.; Rothman, J. E. SNAREpins: minimal machinery for membrane fusion. Cell 1998, 92, 759-772.

(16) Cypionka, A.; Stein, A.; Hernandez, J. M.; Hippchen, H.; Jahn, R.; Walla, P. J. Discrimination between docking and fusion of liposomes reconstituted with neuronal SNARE-proteins using FCS. Proc. Natl. Acad. Sci. USA 2009, 106, 18575-18580.

(17) Hernandez, J. M.; Stein, A.; Behrmann, E.; Riedel, D.; Cypionka, A.; Farsi, Z.; Walla, P. J.; Raunser, S.; Jahn, R. Membrane fusion intermediates via directional and full assembly of the SNARE complex. Science 2012, 336, 1581-1584.

(18) Brunger, A. T.; Weninger, K.; Bowen, M.; Chu, S. Single-molecule studies of the neuronal SNARE fusion machinery. Annu. Rev. Biochem. 2009, 78, 903-928.

(19) Yoon, T.-Y.; Okumus, B.; Zhang, F.; Shin, Y.-K.; Ha, T. Multiple intermediates in SNARE-induced membrane fusion. Proc. Natl. Acad. Sci. USA 2006, 103, 19731-19736.

(20) Diao, J.; Ishitsuka, Y.; Lee, H.; Joo, C.; Su, Z.; Syed, S.; Shin, Y.-K.; Yoon, T.-Y.; Ha, T. A single vesicle-vesicle fusion assay for in vitro studies of SNAREs and accessory proteins. Nat. Protoc. 2012, 7, 921-934.

(21) Kyoung, M.; Zhang, Y.; Diao, J.; Chu, S.; Brunger, A. T. Studying calcium-triggered vesicle fusion in a single vesicle-vesicle content and lipid-mixing system. Nat. Protoc. 2013, 8, 1-16. 
(22) Richmond, D. L.; Schmid, E. M.; Martens, S.; Stachowiak, J. C.; Liska, N.; Fletcher, D. A. Forming giant vesicles with controlled membrane composition, asymmetry, and contents. Proc. Natl. Acad. Sci. USA 2011, 108, 9431-9436.

(23) Karatekin, E.; Di Giovanni, J.; Iborra, C.; Coleman, J.; O'Shaughnessy, B.; Seagar, M.; Rothman, J. E. A fast, single-vesicle fusion assay mimics physiological SNARE requirements. Proc. Natl. Acad. Sci. USA 2010, 107, 3517-3521.

(24) Karatekin, E.; Rothman, J. E. Fusion of single proteoliposomes with planar, cushioned bilayers in microfluidic flow cells. Nat. Protoc. 2012, 7, 903-920.

(25) van den Bogaart, G.; Holt, M. G.; Bunt, G.; Riedel, D.; Wouters, F. S.; Jahn, R. One SNARE complex is sufficient for membrane fusion. Nat. Struct. Mol. Biol. 2010, 17, 358-364.

(26) Hernandez, J. M.; Kreutzberger, A. J.; Kiessling, V.; Tamm, L. K.; Jahn, R. Variable cooperativity in SNARE-mediated membrane fusion. Proc. Natl. Acad. Sci. USA 2014, $111,12037-12042$.

(27) Schneggenburger, R.; Neher, E. Intracellular calcium dependence of transmitter release rates at a fast central synapse. Nature 2000, 406, 889-893.

(28) Schneggenburger, R.; Neher, E. Presynaptic calcium and control of vesicle fusion. Curr. Opin. Neurobiol. 2005, 15, 266-274.

(29) Smith, C. U. M. G-Protein-Coupled Receptors. In Elements of Molecular Neurobiology; 3 ed.; John Wiley \& Sons, Ltd, 2003; pp 167-196.

(30) Smith, C. U. M. Sensory Transduction. In Elements of Molecular Neurobiology; 3 ed.; John Wiley \& Sons, Ltd, 2003; pp 286-318.

(31) Brose, N.; Petrenko, A. G.; Südhof, T. C.; Jahn, R. Synaptotagmin: a calcium sensor on the synaptic vesicle surface. Science 1992, 256, 1021-1025.

(32) Chapman, E. R. How does synaptotagmin trigger neurotransmitter release? Annu. Rev. Biochem. 2008, 77, 615-641.

(33) Ubach, J.; Zhang, X.; Shao, X.; Südhof, T. C.; Rizo, J. Ca ${ }^{2+}$ binding to synaptotagmin: how many $\mathrm{Ca}^{2+}$ ions bind to the tip of a $\mathrm{C}_{2}$-domain? EMBO J. 1998, 17, 3921-3930.

(34) Fernandez, I.; Araç, D.; Ubach, J.; Gerber, S. H.; Shin, O.; Gao, Y.; Anderson, R. G.; Südhof, T. C.; Rizo, J. Three-dimensional structure of the synaptotagmin $1 \mathrm{C}_{2} \mathrm{~B}$-domain: synaptotagmin 1 as a phospholipid binding machine. Neuron 2001, 32, 1057-1069.

(35) Honigmann, A.; van den Bogaart, G.; Iraheta, E.; Risselada, H. J.; Milovanovic, D.; Mueller, V.; Müllar, S.; Diederichsen, U.; Fasshauer, D.; Grubmüller, H.; Hell, S. W.; 
Eggeling, C.; Kühnel, K.; Jahn, R. Phosphatidylinositol 4,5-bisphosphate clusters act as molecular beacons for vesicle recruitment. Nat. Struct. Mol. Biol. 2013, 20, 679-686.

(36) Bai, J.; Tucker, W. C.; Chapman, E. R. PIP 2 increases the speed of response of synaptotagmin and steers its membrane-penetration activity toward the plasma membrane. Nat. Struct. Mol. Biol. 2004, 11, 36-44.

(37) Stein, A.; Radhakrishnan, A.; Riedel, D.; Fasshauer, D.; Jahn, R. Synaptotagmin activates membrane fusion through a $\mathrm{Ca}^{2+}$-dependent trans interaction with phospholipids. Nat. Struct. Mol. Biol. 2007, 14, 904-911.

(38) Wang, Z.; Liu, H.; Gu, Y.; Chapman, E. R. Reconstituted synaptotagmin I mediates vesicle docking, priming, and fusion. J. Cell Biol. 2011, 195, 1159-1170.

(39) Lee, H.-K.; Yang, Y.; Su, Z.; Hyeon, C.; Lee, T. S.; Lee, H. W.; Kweon, D.-H.; Shin, Y.-K.; Yoon, T.-Y. Dynamic $\mathrm{Ca}^{2+}$-dependent stimulation of vesicle fusion by membrane-anchored synaptotagmin 1 . Science 2010, 328, 760-763.

(40) Chicka, M. C.; Hui, E.; Liu, H.; Chapman, E. R. Synaptotagmin arrests the SNARE complex before triggering fast, efficient membrane fusion in response to $\mathrm{Ca}^{2+}$. Nat . Struct. Mol. Biol. 2008, 15, 827-835.

(41) Yang, X.; Kaeser-Woo, Y. J.; Pang, Z. P.; Xu, W.; Südhof, T. C. Complexin clamps asynchronous release by blocking a secondary $\mathrm{Ca}^{2+}$ sensor via its accessory $\alpha$ helix. Neuron 2010, 68, 907-920.

(42) Pobbati, A. V.; Stein, A.; Fasshauer, D. N- to C-terminal SNARE complex assembly promotes rapid membrane fusion. Science 2006, 313, 673-676.

(43) van den Bogaart, G.; Thutupalli, S.; Risselada, J. H.; Meyenberg, K.; Holt, M.; Riedel, D.; Diederichsen, U.; Herminghaus, S.; Grubmüller, H.; Jahn, R. Synaptotagmin-1 may be a distance regulator acting upstream of SNARE nucleation. Nat. Struct. Mol. Biol. 2011, 18, 805-812.

(44) Perin, M. S.; Brose, N.; Jahn, R.; Südhof, T. C. Domain structure of synaptotagmin (p65). J. Biol. Chem. 1991, 266, 623-629.

(45) Pawlicki, M.; Collins, H. A.; Denning, R. G.; Anderson, H. L. Two-photon absorption and the design of two-photon dyes. Angew. Chem. Int. Ed. 2009, 48, 3244-3266.

(46) Krieger, J. W.; Langowski, J. QuickFit 3.0 (status: beta, compiled: 10.12.2012, SVN: 1959): a data evaluation application for biophysics. http://www.dkfz.de/ Macromol/quickfit/, 2011.

(47) Becker, W. The bh TCSPC Handbook. 5 ed., 2012. 
(48) Heinze, K. G.; Koltermann, A.; Schwille, P. Simultaneous two-photon excitation of distinct labels for dual-color fluorescence crosscorrelation analysis. Proc. Natl. Acad. Sci. USA 2000, 97, 10377-10382.

(49) Schwille, P.; Haupts, U.; Maiti, S.; Webb, W. W. Molecular dynamics in living cells observed by fluorescence correlation spectroscopy with one- and two-photon excitation. Biophys. J. 1999, 77, 2251-2265.

(50) Lakowicz, J. R. Fluorescence Correlation Spectroscopy. In Principles of Fluorescence Spectroscopy; 3 ed.; Springer US, 2006; pp 797-840.

(51) Rigaud, J. L.; Pitard, B.; Levy, D. Reconstitution of membrane proteins into liposomes: application to energy-transducing membrane-proteins. Biochim. Biophys. Acta Bioenergetics 1995, 1231, 223-246.

(52) Fluo Calcium Indicators. Life Technologies, 2011.

(53) Chung, M.; Koo, B. J.; Boxer, S. G. Formation and analysis of topographical domains between lipid membranes tethered by DNA hybrids of different lengths. Faraday Discuss. 2013, 161, 333-345.

(54) Wachowius, F.; Javadi-Zarnaghi, F.; Höbartner, C. Combinatorial mutation interference analysis reveals functional nucleotides required for DNA catalysis. Angew. Chem. Int. Ed. 2010, 49, 8504-8508.

(55) Chan, Y. H.; van Lengerich, B.; Boxer, S. G. Lipid-anchored DNA mediates vesicle fusion as observed by lipid and content mixing. Biointerphases 2008, 3, FA17-FA21.

(56) Lu, X.; Zhang, F.; McNew, J. A.; Shin, Y.-K. Membrane fusion induced by neuronal SNAREs transits through hemifusion. J. Biol. Chem. 2005, 280, 30538-30541.

(57) Stengel, G.; Zahn, R.; Höök, F. DNA-induced programmable fusion of phospholipid vesicles. J. Am. Chem. Soc. 2007, 129, 9584-9585.

(58) Thiol-Reactive Probe Labeling Protocol. Life Technologies, 2006.

(59) McIntyre, J. C.; Sleight, R. G. Fluorescence assay for phospholipid membrane asymmetry. Biochemistry 1991, 30, 11819-11827.

(60) Park, K. K.; Han, S. Y.; Lim, H. S. Reduction of N-arylmaleimides with sodium dithionite: observation of dimeric products. Bull. Korean Chem. Soc. 1997, 18, 11451146.

(61) McDonald, J. C.; Duffy, D. C.; Anderson, J. R.; Chiu, D. T.; Wu, H. K.; Schueller, O. J. A.; Whitesides, G. M. Fabrication of microfluidic systems in poly(dimethylsiloxane). Electrophoresis 2000, 21, 27-40. 
(62) Lin, C.-C.; Seikowski, J.; Pérez-Lara, A.; Jahn, R.; Höbartner, C.; Walla, P. J. Control of membrane gaps by synaptotagmin- $\mathrm{Ca}^{2+}$ measured with a novel membrane distance ruler. Nat. Commun. 2014, 5, 5859.

(63) Vrljic, M.; Strop, P.; Ernst, J. A.; Sutton, R. B.; Chu, S.; Brunger, A. T. Molecular mechanism of the synaptotagmin-SNARE interaction in $\mathrm{Ca}^{2+}$-triggered vesicle fusion. Nat. Struct. Mol. Biol. 2010, 17, 325-331.

(64) Choi, U. B.; Strop, P.; Vrljic, M.; Chu, S.; Brunger, A. T.; Weninger, K. R. Singlemolecule FRET-derived model of the synaptotagmin 1-SNARE fusion complex. Nat. Struct. Mol. Biol. 2010, 17, 318-324.

(65) Baumann, C. G.; Smith, S. B.; Bloomfield, V. A.; Bustamante, C. Ionic effects on the elasticity of single DNA molecules. Proc. Natl. Acad. Sci. USA 1997, 94, 6185-6190.

(66) Chung, M.; Lowe, R. D.; Chan, Y.-H.; Ganesan, P. V.; Boxer, S. G. DNA-tethered membranes formed by giant vesicle rupture. J. Struct. Biol. 2009, 168, 190-199.

Vennekate, W.; Schröder, S.; Lin, C.-C.; van den Bogaart, G.; Grunwald, M.; Jahn, R.; Walla, P. J. Cis- and trans-membrane interactions of synaptotagmin-1. Proc. Natl. Acad. Sci. USA 2012, 109, 11037-11042.

(68) McLaughlin, S.; Wang, J.; Gambhir, A.; Murray, D. PIP 2 and proteins: interactions, organization, and information flow. Annu. Rev. Biophys. Biomol. Struct. 2002, 31, 151175.

(69) Lai, Y.; Lou, X.; Jho, Y.; Yoon, T.-Y.; Shin, Y.-K. The synaptotagmin 1 linker may function as an electrostatic zipper that opens for docking but closes for fusion pore opening. Biochem. J. 2013, 456, 25-33.

(70) Lu, B.; Kiessling, V.; Tamm, L. K.; Cafiso, D. S. The juxtamembrane linker of fulllength synaptotagmin 1 controls oligomerization and calcium-dependent membrane binding. J. Biol. Chem. 2014, 289, 22161-22171.

(71) Lai, Y.; Shin, Y.-K. The importance of an asymmetric distribution of acidic lipids for synaptotagmin 1 function as a $\mathrm{Ca}^{2+}$ sensor. Biochem. J. 2012, 443, 223-229.

(72) Smith, C. U. M. Biomembranes. In Elements of Molecular Neurobiology; John Wiley \& Sons, Ltd, 2003; pp 140-166.

(73) Araç, D.; Chen, X.; Khant, H. A.; Ubach, J.; Ludtke, S. J.; Kikkawa, M.; Johnson, A. E.; Chiu, W.; Südhof, T. C.; Rizo, J. Close membrane-membrane proximity induced by $\mathrm{Ca}^{2+}$-dependent multivalent binding of synaptotagmin-1 to phospholipids. Nat. Struct. Mol. Biol. 2006, 13, 209-217. 
(74) Hui, E.; Bai, J.; Chapman, E. R. $\mathrm{Ca}^{2+}$-triggered simultaneous membrane penetration of the tandem C2-domains of synaptotagmin I. Biophys. J. 2006, 91, 1767-1777.

(75) van den Bogaart, G.; Meyenberg, K.; Risselada, H. J.; Amin, H.; Willig, K. I.; Hubrich, B. E.; Dier, M.; Hell, S. W.; Grubmüller, H.; Diederichsen, U.; Jahn, R. Membrane protein sequestering by ionic protein-lipid interactions. Nature 2011, 479, 552-555.

(76) Aoyagi, K.; Sugaya, T.; Umeda, M.; Yamamoto, S.; Terakawa, S.; Takahashi, M. The activation of exocytotic sites by the formation of phosphatidylinositol 4,5-bisphosphate microdomains at syntaxin clusters. J. Biol. Chem. 2005, 280, 17346-17352.

Bharat, T. A. M.; Malsam, J.; Hagen, W. J. H.; Scheutzow, A.; Söllner, T. H.; Briggs, J. A. G. SNARE and regulatory proteins induce local membrane protrusions to prime docked vesicles for fast calcium-triggered fusion. EMBO Rep. 2014, 15, 308-314.

(78) Ellena, J. F.; Liang, B.; Wiktor, M.; Stein, A.; Cafiso, D. S.; Jahn, R.; Tamm, L. K. Dynamic structure of lipid-bound synaptobrevin suggests a nucleation-propagation mechanism for trans-SNARE complex formation. Proc. Natl. Acad. Sci. USA 2009, 106, 20306-20311.

(79) Hanson, P. I.; Roth, R.; Morisaki, H.; Jahn, R.; Heuser, J. E. Structure and conformational changes in NSF and its membrane receptor complexes visualized by quick-freeze/deep-etch electron microscopy. Cell 1997, 90, 523-535.

(80) Gao, Y.; Zorman, S.; Gundersen, G.; Xi, Z.; Ma, L.; Sirinakis, G.; Rothman, J. E.; Zhang, Y. Single reconstituted neuronal SNARE complexes zipper in three distinct stages. Science 2012, 337, 1340-1343.

(81) Seven, A. B.; Brewer, K. D.; Shi, L.; Jiang, Q.-X.; Rizo, J. Prevalent mechanism of membrane bridging by synaptotagmin-1. Proc. Natl. Acad. Sci. USA 2013, 110, E3243E3252.

(82) Connell, E.; Giniatullina, A.; Lai-Kee-Him, J.; Tavare, R.; Ferrari, E.; Roseman, A.; Cojoc, D.; Brisson, A. R.; Davletov, B. Cross-linking of phospholipid membranes is a conserved property of calcium-sensitive synaptotagmins. J. Mol. Biol. 2008, 380, 42-50.

(83) Herrick, D. Z.; Kuo, W.; Huang, H.; Schwieters, C. D.; Ellena, J. F.; Cafiso, D. S. Solution and membrane-bound conformations of the tandem C2A and C2B domains of synaptotagmin 1: evidence for bilayer bridging. J. Mol. Biol. 2009, 390, 913-923.

(84) Kuo, W.; Herrick, D. Z.; Cafiso, D. S. Phosphatidylinositol 4,5-bisphosphate alters synaptotagmin 1 membrane docking and drives opposing bilayers closer together. Biochemistry 2011, 50, 2633-2641. 
(85) Lai, A. L.; Huang, H.; Herrick, D. Z.; Epp, N.; Cafiso, D. S. Synaptotagmin 1 and SNAREs form a complex that is structurally heterogeneous. J. Mol. Biol. 2011, 405, 696-706.

(86) Liu, H.; Bai, H.; Xue, R.; Takahashi, H.; Edwardson, J. M.; Chapman, E. R. Linker mutations reveal the complexity of synaptotagmin 1 action during synaptic transmission. Nat. Neurosci. 2014, 17, 670-677.

(87) Mackler, J. M.; Drummond, J. A.; Loewen, C. A.; Robinson, I. M.; Reist, N. E. The $\mathrm{C}_{2} \mathrm{~B} \mathrm{Ca}^{2+}$-binding motif of synaptotagmin is required for synaptic transmission in vivo. Nature 2002, 418, 340-344.

(88) Nishiki, T.; Augustine, G. J. Dual roles of the $\mathrm{C}_{2} \mathrm{~B}$ domain of synaptotagmin $\mathrm{I}$ in synchronizing $\mathrm{Ca}^{2+}$-dependent neurotransmitter release. J. Neurosci. 2004, 24, 85428550.

(89) Martens, S.; Kozlov, M. M.; McMahon, H. T. How synaptotagmin promotes membrane fusion. Science 2007, 316, 1205-1208.

(90) Hui, E.; Johnson, C. P.; Yao, J.; Dunning, F. M.; Chapman, E. R. Synaptotagminmediated bending of the target membrane is a critical step in $\mathrm{Ca}^{2+}$-regulated fusion. Cell 2009, 138, 709-721.

(91) Striegel, A. R.; Biela, L. M.; Evans, C. S.; Wang, Z.; Delehoy, J. B.; Sutton, R. B.; Chapman, E. R.; Reist, N. E. Calcium binding by synaptotagmin's $\mathrm{C}_{2} \mathrm{~A}$ domain is an essential element of the electrostatic switch that triggers synchronous synaptic transmission. J. Neurosci. 2012, 32, 1253-1260.

(92) Saff, E. B.; Kuijlaars, A. B. J. Distributing many points on a sphere. Math. Intell. 1997, 19, 5-11.

(93) Kučerka, N.; Tristram-Nagle, S.; Nagle, J. F. Structure of fully hydrated fluid phase lipid bilayers with monounsaturated chains. J. Membr. Biol. 2005, 208, 193-202.

(94) Walla, P. J.; Yom, J.; Krueger, B. P.; Fleming, G. R. Two-photon excitation spectrum of light-harvesting complex II and fluorescence upconversion after one- and two-photon excitation of the carotenoids. J. Phys. Chem. B 2000, 104, 4799-4806.

(95) Mottram, L. F.; Boonyarattanakalin, S.; Kovel, R. E.; Peterson, B. R. The Pennsylvania green fluorophore: A hybrid of Oregon Green and Tokyo Green for the construction of hydrophobic and pH-insensitive molecular probes. Org. Lett. 2006, 8, 581-584. 
(96) van der Veen, R. M.; Cannizzo, A.; van Mourik, F.; Vlček, A.; Chergui, M. Vibrational relaxation and intersystem crossing of binuclear metal complexes in solution. J. Am. Chem. Soc. 2011, 133, 305-315.

(97) Jarzęba, W.; Walker, G. C.; Johnson, A. E.; Kahlow, M. A.; Barbara, P. F. Femtosecond microscopic solvation dynamics of aqueous solutions. J. Phys. Chem. 1988, 92, 7039-7041.

(98) Jimenez, R.; Fleming, G. R.; Kumar, P. V.; Maroncelli, M. Femtosecond solvation dynamics of water. Nature 1994, 369, 471-473.

(99) Barucha-Kraszewska, J.; Kraszewski, S.; Jurkiewicz, P.; Ramseyer, C.; Hof, M. Numerical studies of the membrane fluorescent dyes dynamics in ground and excited states. Biochim. Biophys. Acta - Biomembranes 2010, 1798, 1724-1734.

(100) Gerecke, M.; Pauli, J.; Ernsting, N. P. Dynamic fluorescence Stokes Shift near phospholipid bilayers for complete environmental response. In XXV IUPAC Symposium on Photochemistry: Bordeaux, France, 2014.

(101) Chattopadhyay, A.; Mukherjee, S. Fluorophore environments in membrane-bound probes: a red edge excitation shift study. Biochemistry 1993, 32, 3804-3811.

(102) van Stokkum, I. H. M.; Larsen, D. S.; van Grondelle, R. Global and target analysis of time-resolved spectra. Biochim. Biophys. Acta - Bioenergetics 2004, 1657, 82-104.

(103) Hsieh, C.-C.; Chen, K.-Y.; Hsieh, W.-T.; Lai, C.-H.; Shen, J.-Y.; Jiang, C.-M.; Duan, H.-S.; Chou, P.-T. Cyano analogues of 7-azaindole: probing excited-state chargecoupled proton transfer reactions in protic solvents. ChemPhysChem 2008, 9, 22212229.

(104) Lygina, A. S.; Meyenberg, K.; Jahn, R.; Diederichsen, U. Transmembrane domain peptide/peptide nucleic acid hybrid as a model of a SNARE protein in vesicle fusion. Angew. Chem. Int. Ed. 2011, 50, 8597-8601.

(105) Wilhelm, B. G.; Mandad, S.; Truckenbrodt, S.; Kröhnert, K.; Schäfer, C.; Rammner, B.; Koo, S. J.; Claßen, G. A.; Krauss, M.; Haucke, V.; Urlaub, H.; Rizzoli, S. O. Composition of isolated synaptic boutons reveals the amounts of vesicle trafficking proteins. Science 2014, 344, 1023-1028.

(106) Rizzoli, S. O.; Betz, W. J. The structural organization of the readily releasable pool of synaptic vesicles. Science 2004, 303, 2037-2039.

(107) Martens, S.; McMahon, H. T. Mechanisms of membrane fusion: disparate players and common principles. Nat. Rev. Mol. Cell Biol. 2008, 9, 543-556. 
(108) Sapir, A.; Avinoam, O.; Podbilewicz, B.; Chernomordik, L. V. Viral and developmental cell fusion mechanisms: conservation and divergence. Dev. Cell 2008, 14, 11-21.

(109) Zhao, L.; Lustres, J. L. P.; Farztdinov, V.; Ernsting, N. P. Femtosecond fluorescence spectroscopy by upconversion with tilted gate pulses. Phys. Chem. Chem. Phys. 2005, 7, 1716-1725.

(110) Zhang, X. X.; Wurth, C.; Zhao, L.; Resch-Genger, U.; Ernsting, N. P.; Sajadi, M. Femtosecond broadband fluorescence upconversion spectroscopy: Improved setup and photometric correction. Rev. Sci. Instrum. 2011, 82, 063108.

(111) Chou, P.-T.; Chi, Y.; Chung, M.-W.; Lin, C.-C. Harvesting luminescence via harnessing the photophysical properties of transition metal complexes. Coord. Chem. Rev. 2011, 255, 2653-2665.

(112) Rohan, J. G.; Citron, Y. R.; Durrell, A. C.; Cheruzel, L. E.; Gray, H. B.; Grubbs, R. H.; Humayun, M.; Engisch, K. L.; Pikov, V.; Chow, R. H. Light-triggered modulation of cellular electrical activity by ruthenium diimine nanoswitches. ACS Chem. Neurosci. 2013, 4, 585-593.

(113) Zhang, S.; Hosaka, M.; Yoshihara, T.; Negishi, K.; Iida, Y.; Tobita, S.; Takeuchi, T. Phosphorescent light-emitting iridium complexes serve as a hypoxia-sensing probe for tumor imaging in living animals. Cancer Res. 2010, 70, 4490-4498.

(114) Lin, C.-C.; Chen, C.-L.; Chung, M.-W.; Chen, Y.-J.; Chou, P.-T. Effects of multibranching on 3-hydroxyflavone-based chromophores and the excited-state intramolecular proton transfer dynamics. J. Phys. Chem. A 2010, 114, 10412-10420.

(115) Das, R.; Klymchenko, A. S.; Duportail, G.; Mely, Y. Excited state proton transfer and solvent relaxation of a 3-hydroxyflavone probe in lipid bilayers. J. Phys. Chem. B 2008, 112, 11929-11935.

(116) Yoshihara, T.; Yamaguchi, Y.; Hosaka, M.; Takeuchi, T.; Tobita, S. Ratiometric molecular sensor for monitoring oxygen levels in living cells. Angew. Chem. Int. Ed. 2012, 51, 4148-4151.

(117) Liao, P.-N.; Pillai, S.; Gust, D.; Moore, T. A.; Moore, A. L.; Walla, P. J. Two-photon study on the electronic interactions between the first excited singlet states in carotenoidtetrapyrrole dyads. J. Phys. Chem. A 2011, 115, 4082-4091.

(118) McNew, J. A.; Parlati, F.; Fukuda, R.; Johnston, R. J.; Paz, K.; Paumet, F.; Söllner, T. H.; Rothman, J. E. Compartmental specificity of cellular membrane fusion encoded in SNARE proteins. Nature 2000, 407, 153-159. 
(119) Zwilling, D.; Cypionka, A.; Pohl, W. H.; Fasshauer, D.; Walla, P. J.; Wahl, M. C.; Jahn, R. Early endosomal SNAREs form a structurally conserved SNARE complex and fuse liposomes with multiple topologies. EMBO J. 2007, 26, 9-18.

(120) Golebiewska, U.; Nyako, M.; Woturski, W.; Zaitseva, I.; McLaughlin, S. Diffusion coefficient of fluorescent phosphatidylinositol 4,5-bisphosphate in the plasma membrane of cells. Mol. Biol. Cell 2008, 19, 1663-1669.

(121) Mueller, V.; Ringemann, C.; Honigmann, A.; Schwarzmann, G.; Medda, R.; Leutenegger, M.; Polyakova, S.; Belov, V. N.; Hell, S. W.; Eggeling, C. STED nanoscopy reveals molecular details of cholesterol- and cytoskeleton-modulated lipid interactions in living cells. Biophys. $J$ 2011, 101, 1651-1660. 
6 APPENDICES 


\section{CURRICULUM VITAE}

\section{Education and Work Experiences}

- 10/2011 present $\quad$ : PhD at MPI-BPC. Supervisor: Prof. Dr. Peter Jomo Walla.

- 01/2011 07/2011 : Research assistant in the lab of Prof. Dr. Pi-Tai Chou.

- 09/2008 07/2010 : M.S., National Taiwan University, Department of Chemistry. Supervisor: Prof. Dr. Pi-Tai Chou. Thesis title: Photophysics of transition metal complexes and two-photon absorbing chromophores.

- 09/2004 06/2008 ～: B.S., National Taiwan University, Department of Chemistry.

\section{Fellowships and Awards}

- 04/2014: Selected talk at the GDCh $6^{\text {th }}$ Braunschweiger Jungchemiker Tagung 2015.

- 10/2014: Travel grant for poster presentation at the $50^{\text {th }}$ Anniversary of the Heinrich Wieland Prize Scientific Symposium.

- 07/2014: Poster Award at the XXVth IUPAC Symposium on Photochemistry, Bordeaux.

- 07/2013: Participant of the $63^{\text {rd }}$ Lindau Nobel Laureate Meeting Chemistry, nominated by MPI-BPC.

- 06/2012: Stipend of IMPRS for Physics of Biological and Complex Systems (evaluated and extended in 06/2014).

- 04/2012: DAAD Research Grants for Doctoral Candidates and Young Academics and Scientists.

- 12/2010: Annual Meeting of the Chinese Chemical Society Dissertation Award. 


\section{PUBLICATIONS}

i. $\quad$ Lin, C.-C.; Seikowski, J.; Pérez-Lara, A.; Jahn, R.; Höbartner, C.; Walla, P. J.: Control of membrane gaps by synaptotagmin- $\mathrm{Ca}^{2+}$ measured with a novel membrane distance ruler. Nat. Commun. 2014, 5, 5859 (doi: 10.1038/ncomms6859).

\section{Author contributions:}

C.-C.L., R.J. and P.J.W. wrote the paper. J.S. and C.H. synthesized the DNA lipids. A.P.-L. and R.J. provided the proteins. C.-C.L. designed the study and performed all other experiments. All authors discussed the results and commented on the manuscript.

ii. Vennekate, W.; Schröder, S.; Lin, C.-C.; van den Bogaart, G.; Grunwald, M.; Jahn, R.; Walla, P. J.: Cis- and trans-membrane interactions of synaptotagmin-1. Proc. Natl. Acad. Sci. USA 2012, 109, 11037-11042.

\section{Author contributions:}

R.J. and P.J.W. designed research; W.V., S.S., and C.-C.L. performed research; G.v.d.B. and M.G. contributed new reagents/analytic tools; W.V., S.S., and C.-C.L. analyzed data; and W.V., G.v.d.B., R.J., and P.J.W. wrote the paper.

iii. $\quad$ Lin, C.-C.; Hsu, H.-F.; Bodenschatz, E.; Jahn, R.; Walla, P. J.: Asymetrically labeled liposomes as a new tool to study membrane fusion. Manuscript in preparation. 